\title{
Implications in the treatment of peripheral arterial disease
}

Citation for published version (APA):

Bolt, L. J. J. (2022). Implications in the treatment of peripheral arterial disease: focussing on endovascular and non-invasive strategies. [Doctoral Thesis, Maastricht University]. Ipskamp Printing BV. https://doi.org/10.26481/dis.20220331lb

Document status and date:

Published: 01/01/2022

DOI:

$10.26481 /$ dis.20220331lb

Document Version:

Publisher's PDF, also known as Version of record

\section{Please check the document version of this publication:}

- A submitted manuscript is the version of the article upon submission and before peer-review. There can be important differences between the submitted version and the official published version of record.

People interested in the research are advised to contact the author for the final version of the publication, or visit the DOI to the publisher's website.

- The final author version and the galley proof are versions of the publication after peer review.

- The final published version features the final layout of the paper including the volume, issue and page numbers.

Link to publication

\footnotetext{
General rights rights.

- You may freely distribute the URL identifying the publication in the public portal. please follow below link for the End User Agreement:

www.umlib.nl/taverne-license

Take down policy

If you believe that this document breaches copyright please contact us at:

repository@maastrichtuniversity.nl

providing details and we will investigate your claim.
}

Copyright and moral rights for the publications made accessible in the public portal are retained by the authors and/or other copyright owners and it is a condition of accessing publications that users recognise and abide by the legal requirements associated with these

- Users may download and print one copy of any publication from the public portal for the purpose of private study or research.

- You may not further distribute the material or use it for any profit-making activity or commercial gain

If the publication is distributed under the terms of Article $25 \mathrm{fa}$ of the Dutch Copyright Act, indicated by the "Taverne" license above, 


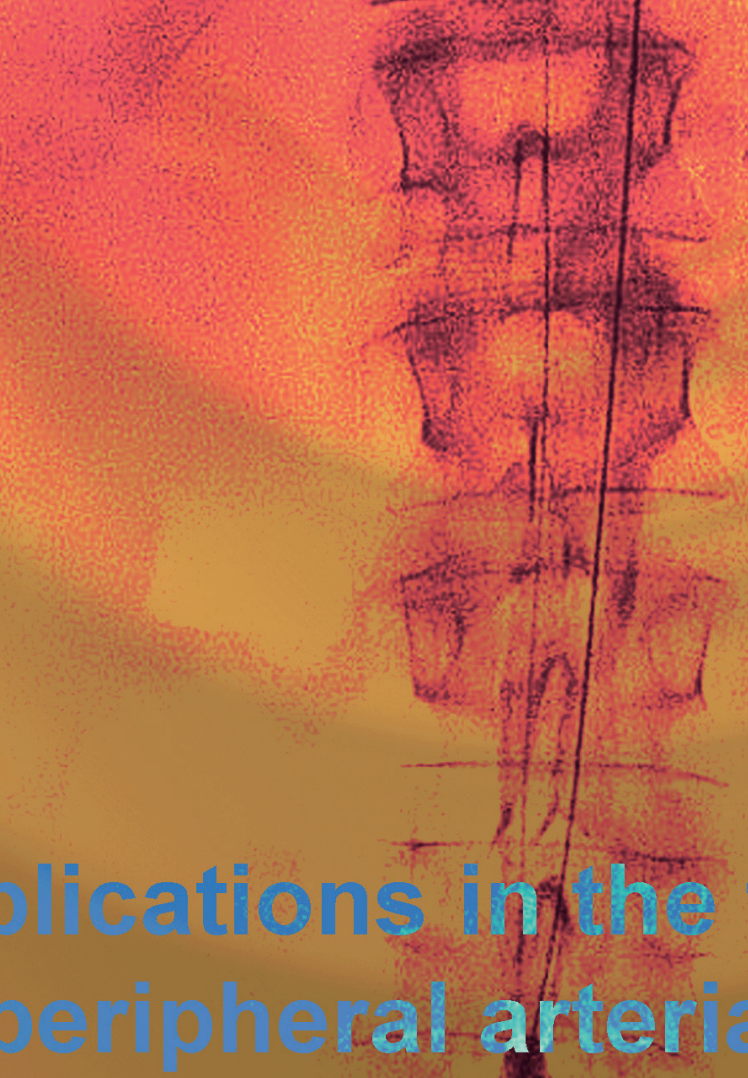

\section{Focussing on endovascular and non-invasive strategies}

Lars Bolt 

Implications in the treatment of peripheral arterial disease

Focussing on endovascular and non-invasive strategies 


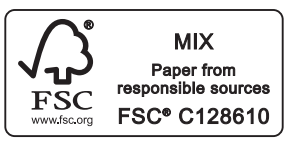

ISBN: 978-94-6421-668-4

Image on cover: Remco Wetzels

Layout: Wendy Bour-van Telgen

Printing: Ipskamp Printing

Publication of this thesis was financially supported by: $\mathrm{SBOH}$

\section{SBCHK \\ voor artsen in opleiding}

\section{(c) 2022 L.J.J. Bolt}

All rights reserved. No part of this thesis may be reproduced or transmitted in any form, by any means, electronic or mechanical, without the prior permission of the author, or where appropriate, of the publisher of the articles. 


\title{
Implications in the treatment of peripheral arterial disease
}

\section{Focussing on endovascular and non-invasive strategies}

\author{
Proefschrift \\ ter verkrijging van de graad van doctor aan de Universiteit Maastricht, \\ op gezag van Rector Magnificus prof.dr. Rianne M. Letschert, \\ volgens het besluit van het College van Decanen, \\ in het openbaar te verdedigen op \\ donderdag 31.03.2022 om 10:00 uur
}

door

Lars Johannes Jerome Bolt

Geboren op 8 mei 1987

te Groningen 


\section{Promotor}

Prof. dr. G.W.H. Schurink, Hoogleraar vaatchirurgie,

MUMC, Universiteit Maastricht

\section{Co-promotor}

Dr. L.H. Bouwman, Zuyderland Medisch Centrum

\section{Beoordelingscommissie}

Prof. dr. J.W.M. Muris, Hoogleraar Huisartsgeneeskunde,

Universiteit Maastricht (voorzitter)

Prof. dr. A. W.J. van 't Hof, Hoogleraar Interventiecardiologie,

Universiteit Maastricht

Prof. dr. J.A. van Herwaarden, Hoogleraar Vaatchirurgie: Aorta Pathologie,

Universitair Medisch Centrum Utrecht

Dr. J. Heyligers, Afdeling Chirurgie,

Elisabeth-TweeSteden Ziekenhuis, Tilburg 
Voor Kirsten, Mads, Mette en ... 


\section{Contents}

$\begin{array}{lll}\text { Chapter } 1 \text { General introduction } & 8\end{array}$

1.1 Peripheral arterial disease 9

1.2 Diagnosis and classification 10

1.3 Medical therapy 11

1.4 Supervised exercise therapy 11

1.5 Endovascular revascularization 12

1.6 Radiation 12

1.7 Contrast induced nephropathy 13

$\begin{array}{lll}1.8 \text { Biomarkers } & 14\end{array}$

1.9 Aims and outline of these thesis 14

$\begin{array}{ll}\text { Part } 1 \text { - Implications in endovascular treatment strategies } & 23\end{array}$

Chapter 2 Loss of kidney function after endovascular treatment of 24 peripheral arterial disease

Chapter 3 Loss of kidney function in patients with critical limb ischemia 42 treated endovascular or surgically

Chapter 4 Radiation exposure during percutaneous transluminal $\quad 58$ angioplasty for symptomatic peripheral arterial disease

Chapter 5 Duplex-guided versus conventional percutaneous transluminal angioplasty of iliac TASC II A and B lesions: A randomized controled trial

Chapter 6 Renal decline after endovascular Intervention for Peripheral 90 arterial disease: lodine contrast PTA vs. duplex-guided PTA in TASC II A and B iliac stenotic Leasions 
Chapter 7 Prevention of postcontrast acute kidney injury after percutaneous transluminal angioplasty by inducing RenalGuard controlled furosemide forced diuresis with matched hydration: study protocol for a randomised controlled trial

Chapter 8 Assessment of behavioural determinants influencing success 128 of supervised exercise therapy in patients with intermittent claudication: A cross sectional survey

Chapter 9 General discussion \& Summary

Chapter 10 Nederlandse samenvatting 163 Valorization 166

Dankwoord 169

Curriculum Vitae 174

List of publications 177 List of abbreviations 178 
Genera

introduction 


\subsection{Peripheral arterial disease}

Peripheral arterial disease (PAD) in this manuscript is restricted to atherosclerotic disease of the lower limb arteries. PAD can be either asymptomatic or symptomatic. Asymptomatic patients have a decreased ankle-brachial index (ABI) without complaints of pain in the lower limbs, possible due to inactivity and thus not exerting themselves to such an extent that pain is experienced. Symptomatic PAD often starts with intermittent claudication (IC), where pain is experienced in the calves on exercise and subsides with rest. It is estimated that $9.3 \%$ of patients with asymptomatic PAD, will progress to IC within 5 years' time. ${ }^{1}$ $\mathrm{PAD}$ can progress to critical limb ischemia, where pain occurs in rest and might eventually progress to ulceration, gangrene and possible amputation. Functional impairment due to PAD increases the risk of hospitalization, nursing home placement and mobility loss. ${ }^{2-5}$ Globally it is estimated that approximately 200 million people are affected by PAD. ${ }^{6}$ However this number might be even greater, whereas patient and clinical awareness of the disease is poor. Underdiagnosing PAD is likely, particularly in non-western countries. ${ }^{7}$ The incidence of PAD increases with age, estimated at 5\% at the age of $45-49$ years and increasing to $18 \%$ at the age of $85-89$ years. ${ }^{6}$ The fact that live-expectancy is ever increasing, even among patients with multiple comorbidities, results in a greater increase in incidence. It is well known, that the most important risk-factor in developing PAD is smoking. Smoking increases the risk by two-fold compared to a non-smoker. Furthermore, the greater the amount smoked, the greater the risk. ${ }^{8}$ Another important contributor to the development of PAD is diabetes mellitus (DM). DM also generates a two-fold increased risk in intermittent claudication. However, the relative risk of developing chronic limb ischemia due to DM is much greater, with a fivefold higher risk of major amputation. ${ }^{9}$ Other risk factors are dyslipidaemia, where total cholesterol, low HDL-cholesterol levels and total cholesterol to HDL-cholesterol ratio are the most relevant predictors in PAD. ${ }^{10-12}$ High systolic blood pressure is also a known predictor in the development of PAD, whereas diastolic blood pressure is not. ${ }^{11,12}$ The limitations in mobility and the pain experienced has a great influence on the quality of life in patients diagnosed with PAD. Both asymptomatic and symptomatic PAD have an equal burden as any other cardiovascular disease in the quality of life. ${ }^{13}$ Obviously, patients with CLI experience a heavier burden in quality of life when compared to patients with IC, with regard to discomfort caused by pain. ${ }^{14}$ Although, one might expect a negative progression of the disease over time, subjectively $50 \%$ of patient with IC will experience similar complaints after 2 years and $25 \%$ will even improve. ${ }^{15}$ However when objectively measured, walking ability is often declined. ${ }^{16,17}$ Discrepancy between subjective improvement and objective worsening of walking ability 
might be explained by development of collaterals, decreased neuronal sensitivity due to (diabetic) neuropathy or metabolic adaptation of ischaemic muscles. ${ }^{6}$ Nonetheless, PAD is not to be underestimated, whereas the risk of cardiovascular mortality in patients with an $\mathrm{ABI}<0.90$ is two- to threefold higher when compared to controls. ${ }^{18,19}$

\subsection{Diagnosis and classification}

Ankle-brachial index (ABI) is calculated using the ratio of the highest systolic ankle pressure of either dorsalis pedis artery or posterior tibial artery, and systolic pressure of the brachial artery, in the arm with highest systolic pressure (avoiding bias of a potential subclavian stenosis). Normally the ABI in a healthy individual is $>1$ (1.10-1.25), however an $\mathrm{ABI}>1,4$ is unreliable and can be caused by calcified arteries in progressed PAD. An $\mathrm{ABI}<0,9$ is considered abnormal and indicative of PAD. As a diagnostic tool the $\mathrm{ABI}$ has a sensitivity of $80 \%$ and specificity of $95 \%$. To objectively measure functional limitations in $\mathrm{PAD}$, an exercise treadmill $\mathrm{ABI}$ is measured to establish $\mathrm{PAD}$ in patient with a borderline normal $A B I$ in rest. Whereas $A B I$ will decrease in patients with $P A D$ and keep a steady or increased index in healthy individuals. If an ABI is measured $>1,40$ due to stiff/calcified arteries a TBI (toe-brachial-index) can be of added value. If revascularization is considered, addition imaging is needed to determine location and severity of stenosis or occlusion to determine plan of approach. The modalities used are duplex, CTA and/or MRA. ${ }^{20}$ Classification systems of $\mathrm{PAD}$ are based on either clinical presentation, anatomic distribution of disease or a combination of clinical factors such as presence of infection and wounds. ${ }^{21}$ The American College of Cardiology/American Heart Associations Practice guidelines divides the clinical presentations of PAD in four categories; asymptomatic, intermittent claudication (IC), critical limb ischemia (CLI) and acute limb ischemia (ALI) ${ }^{22,23}$ The first classification based on symptomatic presentation is formulated by the society of cardiovascular surgery in 1952 and published by Fontaine et al..$^{21,24}$ The Fontaine classification divides clinical symptoms in four categories; I asymptomatic, IIa claudication at a distance $>200$ meter, IIb claudication at a distance $<200$ meter, III rest pain, IV necrosis and/or gangrene of the limb. ${ }^{21,24}$ Rutherford adapted the symptomatic classification in 1986 and allocated classification to either acute or chronic limb ischemia. Furthermore, clinical symptoms in chronic limb ischemia were also associated with objective non-invasive measurements such as doppler, arterial-brachial-index and pulse volume recordings. ${ }^{25,26}$ Acute limb ischemia was divided in viable, threatened or irreversible damage to the limb. The Trans-Atlantic Inter-Society Consensus Document II (TASC II) is a consensus formulated in 2000 by vascular surgeons, interventional radiologists 
and cardiologists to provide a recommendations regarding clinical evaluation, diagnosis, treatment, and follow-up in patients with symptomatic PAD. ${ }^{21,27}$ Lesions are divided in the anatomical categories aorto-iliac and femoral popliteal. Classification is distributed in 4 specific groups and are assigned treatment algorithms (endovascular vs surgical). TASC II A lesions have excellent results when treated endovascularly, TASC II B lesions are also recommended to be treated endovascularly, TASC II C have better long-term patency when treated surgically when compared to endovascular management. TASC II D should be treated with open surgery. ${ }^{21}$ However, due to ongoing improvement in endovascular techniques and materials, some TASC II D lesions can potentially by treated endovascularly as an alternative. ${ }^{21,28-30}$

\subsection{Medical therapy}

When diagnosed with PAD, lifestyle changes and structured exercise therapy are needed to limit the progression of the disease. Furthermore, cessation of smoking is paramount, therefor physicians should refer patients to a smoking cessation program or assist in quieting by prescribing pharmacotherapy (nicotine replacement therapy or varenicline) and provide counseling. Optimal glycemic control and antihypertensive therapy are also of great importance in patients with PAD to decrease the risk of a cardiovascular event (Stroke, MI and cardiovascular death). ${ }^{20}$ To further limit the progression, it is recommended to start antiplatelet therapy (aspirin or clopidogrel monotherapy) and statin agents. ${ }^{31-34}$

\subsection{Supervise exercise therapy}

Supervised exercise therapy (SET) is the initial treatment of IC. It is well established that SET is an effective treatment in prolonging peak walking distance (PWD), claudication onset time (COT) and reduce the risk for major cardiovascular event in the future. ${ }^{5,35-37}$ Furthermore, supervise exercise therapy is significantly more effective that unsupervised exercise therapy ${ }^{38,39}$ Patients participating in SET have to exercise under supervision for at least three times per week. Exercise regimen should by tailored, and gradually increased up to an exercise duration of at least 30 minutes. Patients need to be stimulated to walk until near maximal pain, if the pain does not occur within 10 minutes, walking speed or inclination of the treadmill should be adjusted. Stopping when pain arises is allowed, however when the pain is subsided, exercise should be resumed. ${ }^{5}$ Regrettably, SET is often 
inaccessible for patients with PAD due to reimbursement issues and the requirement to travel to an exercise center three times per week. Furthermore, even if SET is reimbursed in a research program, patients still decline participation. ${ }^{40}$ Fifty percent of patients refuse participation due to lack of interest, whereas 19 percent stated that participating in SET is inconvenient. ${ }^{5,40}$ Even though SET is a proven treatment strategy, 2/3th of the patients are not motivated to participate. ${ }^{5,40}$ Whereas SET in not always provided by clinicians due to costs initiating and maintaining SET as a service, clinicians often result to PTA as first treatment. ${ }^{41}$ However, PTA should be reserved as an option to fall back on when unresponsive to SET. ${ }^{37,42}$ Though, with regard to initial treatment, recent studies also demonstrate that the best possible outcome, increasing PWD and COT, is achieved when SET is combined with PTA. ${ }^{35,36}$ It is however discouraged to use PTA as an initial therapy without SET.

\subsection{Endovascular revascularization}

Endovascular revascularization comprises several techniques, namely angioplasty (balloon dilatation), stents and atherectomy. ${ }^{20}$ Improvement of operating skills created a shift to endovascular interventions (opposed to open surgery) and stimulated the continuous evolution of endovascular technology and techniques. Improvement in sheaths, wires, catheters, (cutting) balloons, (covered) stents, drug eluting devices (stents and balloons), and debulking tools created the up rise of the endovascular-first strategy, even in complex TASCII D lesions. ${ }^{20,43}$ Choosing the right technique and approach in the endovascular strategy depends on several lesion characteristics (anatomic location, degree of calcification, lesion length) and skill of the vascular surgeon/interventional radiologist. However, revascularization will only be considered if clinical presentation is objectified by abnormal $\mathrm{ABI}$ and a hemodynamically significant stenosis on imaging, proving limited perfusion to the distal limb.

\subsection{Radiation}

Endovascular revascularization is not without risk. Distal embolization, hematoma, infection, blood loss and arterial injury are few of the possible complications during or after an intervention. Furthermore, there are the deterministic and stochastic effects of ionizing radiation. The short-term deterministic complications such as tissue injury 
(erythema, edema, skin necrosis, ulceration) can occur when exposed to 2 Gy or more. ${ }^{44}$ Whereas stochastic effects, resulting in neoplasms, often take years to reveal and in many occasion are the result of repeated exposure. The risk developing a solid tumor related to radiation is increased up to $42-170 \%$ per sievert. ${ }^{45,46}$ However, long-term cancer risk in the affected population requiring an endovascular intervention is often of little relevance due to advanced age. Guidelines are established by the National Council on Radiation Protection and Measurements (NCRP) to limit radiation dosage used. Dose area product $\left(\mathrm{Gy}^{*} \mathrm{~cm}^{2}\right)$ is considered substantial when $>500 \mathrm{~Gy}$, Skin dose $>3 \mathrm{~Gy}$, fluoroscopy time $>60$ min. ${ }^{46,47}$ Radiation dose is related to several characteristics such as operator skills, patient characteristics, lesion complexity and used equipment. ${ }^{44,48}$ Furthermore, anatomical location plays an important role in radiation dose. Imaging arteries deeply embedded in soft tissue require a greater radiation dosage to penetrate body mass when compared to superficial arteries. ${ }^{44,46,49,50}$ For instance the dosage used in imaging pelvic arteries can be as high as $620 \%$ when compared to below-the-knee interventions. ${ }^{44}$ Moreover, increased BMI can result in an 3-fold increased radiation dosage in endovascular procedures. ${ }^{46,51} \mathrm{Even}$ though clinical relevant radiation effects in the population diagnosed with PAD and treated endovascularly are often limited, it is recommended that the procedure-related radiation dose always is "as low as reasonably achievable" (ALARA).

\subsection{Contrast induced nephropathy}

Conventional endovascular procedures require the use of nephrotoxic iodine contrast, foremost affecting patients with a compromised renal function. Contrast induced nephropathy (CIN), defined as $>25 \%$ increase in serum creatinine or $44 \mathrm{umol} / \mathrm{L}$ within 3 days of intervention, often comes apparent 2-5 days post administration of the iodine contrast. CIN might lead to an increased length of hospital stay and is associated with increased morbidity and mortality. ${ }^{52-54}$ Fortunately, most often decline in renal function will restore within one year to pre-intervention level, less than one percent of patients with CIN will have lasting effects. ${ }^{53,54}$ Independent risk factors in de development of CIN are: heart failure, anemia, critical limb ischemia, diabetes mellitus, over- and underweight. ${ }^{55,56}$ To protect and decrease possibility of CIN, patients with decreased renal function $(<30 \mathrm{ml}$ per $\min / 1.73 \mathrm{~m}^{2}$ ) generally receive pre- and postintervention intravascular volume expansion using isotonic saline. Alternative measures are explored to replace iodine contrast altogether in the prevention of $\mathrm{CIN}$. CO2 is used as contrast agent, however still relying on ionizing radiation. Another solution treating patients endovascularly and 
who are afflicted with chronic renal failure is duplex guided percutaneous endovascular intervention (DuPTA). DuPTA enables performance of an endovascular intervention of the lower extremities, without relying on either ionizing contrast or nephrotoxic iodine contrast.

\subsection{Biomarkers}

CIN is detected 2-5 days after iodine contrast is administered. However, patients treated endovascularly are often discharged one day post intervention. Although patients are requested to assess serum creatinine three days post intervention, this is often dismissed. Therefor CIN might be missed, potentially causing irreversible damage. Moreover, serum creatinine in an healthy person is not suitable as a diagnostic tool, whereas $50 \%$ of the glomerular filtration rate (GFR) must be lost before fluctuations in serum creatinine can be detected. ${ }^{57-59}$ To address these limitations, research was initiated in the past decade regarding the effect of biomarkers in acute kidney injury (AKI). Several promising biomarkers, such as NGAL, TIMP-2, IL-18 are identified enabling us to detect AKI in an early state. ${ }^{57,60-62}$ Moreover, these biomarkers might enable us to identify patients with CIN before discharge and give the opportunity initiate preventive measures to halt further renal decline.

\subsection{Aims and outline of this thesis}

Peripheral arterial disease is increasingly prevalent worldwide, whereas correlated risk behavior is easily accessible, sedentary lifestyle is the norm and life expectancy with multiple comorbidities is increasing. Comorbidities such as CKD and CHF complicate and limit treatment options. Research and resourcefulness are required to optimize result and minimize risk, whereas treatment of PAD is not without risk. In the consideration of treatment strategies, we need to take in account success rate of intervention, side effects and wonder whether the possible benefits outweigh the risks. To optimize result and quality of life it is important to improve a patient's awareness of the disease and make them aware and accountable for possible disease progression and deterioration in functionality and quality of life. The aim of this thesis is to optimize outcome and minimize the risk in the treatment of PAD. Harmful effects of endovascular interventions are established; alternative preventive measures researched and an appeal on a patient's own responsibility is attempted. 
Chapter two evaluates long-term renal function one year after exposure to iodine contrast media due to an endovascular intervention of the lower extremities in patients with intermittent claudication, when compared to a control group participating in supervised exercise therapy.

To evaluate whether endovascular interventions in patient with chronic limb ischemia would also result in long-term loss of kidney function, patients were compared to a comparable population with chronic limb ischemia treated with open surgery in chapter three.

Beside the possible harmful effects of iodine contrast, ionizing radiation is required for imaging of the arteries. The short- and long-term effects of ionizing radiation are well known. Chapter four is dedicated to determine whether anatomic location, patient characteristics and multilevel disease are significantly affecting dose area product, peak skin dose, fluoroscopy time and volume of contrast.

In the attempt to protect the patient against iodine contrast and ionizing radiation a randomized controlled trial was constructed comparing conventional percutaneous endovascular interventions of the iliac arteries to duplex guided percutaneous endovascular intervention (DuPTA). Chapter $\mathbf{5}$ evaluated whether perioperative success of a DuPTA is non-inferior to a conventional procedure in the treatment of iliac TASC II A/B lesions. Chapter $\mathbf{6}$ compares the effect both interventions have on short- and long-term renal function.

Patients with diminished kidney function and in need of an endovascular intervention are treated with hydration therapy. Volume expansion with saline increases diuresis and decreases the toxic effect of iodine contrast. However, there is always the risk of pulmonary edema and electrolyte disturbances. Chapter 7 is a research protocol were the Renalguard is introduced to create furosemide forced diuresis ( $>300 \mathrm{ml} / \mathrm{h}$ ) with direct feedback regulating volume of saline that is administered, creating high renal flow without risk of pulmonary edema. Furthermore, this protocol evaluates whether urine biomarkers are a valid tool in early detection of CIN, when compared to serum creatinine.

However, the best way of treating patients with PAD is to recognize and treat the disease early on. When symptoms aren't progressed to critical limb ischemia, ulceration or necrosis. The first step in treatment of intermittent claudication is supervised exercise 
therapy (SET). It is well known that adherence is low, whereas the therapy in its self can be very successful. In chapter $\mathbf{8}$ a study was performed to pinpoint what causes low adherence. A questionnaire was sent to 200 patients with questions regarding behavior and attitude towards SET, in an attempt to identify what is needed to increase adherence to SET and optimize its result. 


\section{References}

1. Leng GC, Lee AJ, Fowkers FGR, Whiteman M, Dunbar J, Housley E, et al. Incidence, Natural History and Cardiovascular Events in Symptomatic and Asympto matic Peripheral Arterial Disease in the General Population. Int J Epidemiol. 1996 Dec 1;25(6):1172-81.

2. Fried LP, Guralnik JM. Disability in older adults: evidence regarding significance, etiology, and risk. J Am Geriatr Soc. 1997 Jan;45(1):92-100.

3. Guralnik JM, Simonsick EM, Ferrucci L, Glynn RJ, Berkman LF, Blazer DG, et al. A short physical performance battery assessing lower extremity function: association with selfreported disability and prediction of mortality and nursing home admission. J Gerontol. 1994 Mar;49(2):M85-94.

4. Guralnik JM, Ferrucci L, Simonsick EM, Salive ME, Wallace RB. Lower-extremity function in persons over the age of 70 years as a predictor of subsequent disability. $N$ Engl J Med. 1995 Mar 2;332(9):556-61.

5. McDermott MM. Exercise training for intermittent claudication. JVasc Surg. 2017;66(5):161220.

6. Fowkes FGR, Aboyans V, Fowkes FJI, McDermott MM, Sampson UKA, Criqui MH. Peripheral artery disease: epidemiology and global perspectives. Nat Rev Cardiol. 2017 Mar;14(3):156-70.

7. Hirsch AT. Peripheral Arterial Disease Detection, Awareness, and Treatment in Primary Care. JAMA. 2001 Sep 19;286(11):1317.

8. Criqui MH, Aboyans V. Epidemiology of peripheral artery disease. Circ Res. 2015 Apr 24;116(9):1509-26.

9. Jude EB, Oyibo SO, Chalmers N, Boulton AJ. Peripheral arterial disease in diabetic and nondiabetic patients: a comparison of severity and outcome. Diabetes Care. 2001 Aug;24(8):1433-7.

10. Murabito JM, D’Agostino RB, Silbershatz H, Wilson WF. Intermittent claudication. A risk profile from The Framingham Heart Study. Circulation. 1997 Jul 1;96(1):44-9.

11. Meijer WT, Grobbee DE, Hunink MG, Hofman A, Hoes AW. Determinants of peripheral arterial disease in the elderly: the Rotterdam study. Arch Intern Med. 2000 Oct 23;160(19):29348.

12. Newman AB, Siscovick DS, Manolio TA, Polak J, Fried LP, Borhani NO, et al. Ankle-arm index as a marker of atherosclerosis in the Cardiovascular Health Study. Cardiovascular Heart Study (CHS) Collaborative Research Group. Circulation. 1993 Sep;88(3):837-45.

13. Regensteiner JG, Hiatt WR, Coll JR, Criqui MH, Treat-Jacobson D, McDermott MM, et al. The impact of peripheral arterial disease on health-related quality of life in the Peripheral Arterial Disease Awareness, Risk, and Treatment: New Resources for Survival (PARTNERS) Program. Vasc Med Lond Engl. 2008 Feb;13(1):15-24. 
14. Murphy TP. Medical outcomes studies in peripheral vascular disease. J Vasc Interv Radiol JVIR. 1998 Dec;9(6):879-89.

15. Martone VD, Perna S, Cacciatore F, Corrado S, Di Donato A, Di Iorio A, et al. Intermittent Claudication and Risk of Cardiovascular Events. Angiology. 1998 Sep;49(9):843-8.

16. McDermott MM, Guralnik JM, Tian L, Liu K, Ferrucci L, Liao Y, et al. Associations of borderline and low normal ankle-brachial index values with functional decline at 5-year follow-up: the WALCS (Walking and Leg Circulation Study). J Am Coll Cardiol. 2009 Mar 24;53(12):1056-62.

17. McDermott MM, Liu K, Greenland P, Guralnik JM, Criqui MH, Chan C, et al. Functional decline in peripheral arterial disease: associations with the ankle brachial index and leg symptoms. JAMA. 2004 Jul 28;292(4):453-61.

18. Hajibandeh S, Hajibandeh S, Shah S, Child E, Antoniou GA, Torella F. Prognostic significance of ankle brachial pressure index: A systematic review and meta-analysis. Vascular. 2017 Apr;25(2):208-24.

19. Sartipy F, Sigvant B, Lundin F, Wahlberg E. Ten Year Mortality in Different Peripheral Arterial Disease Stages: A Population Based Observational Study on Outcome. Eur J Vasc Endovasc Surg. 2018 Apr;55(4):529-36.

20. Writing Committee Members, Gerhard-Herman MD, Gornik HL, Barrett C, Barshes NR, Corriere MA, et al. 2016 AHA/ACC Guideline on the Management of Patients with Lower Extremity Peripheral Artery Disease: Executive Summary. Vasc Med Lond Engl. 2017;22(3):NP1-43.

21. Hardman RL, Jazaeri O, Yi J, Smith M, Gupta R. Overview of classification systems in peripheral artery disease. Semin Interv Radiol. 2014 Dec;31(4):378-88.

22. Rooke TW, Hirsch AT, Misra S, Sidawy AN, Beckman JA, Findeiss L, et al. Management of patients with peripheral artery disease (compilation of 2005 and 2011 ACCF/AHA Guideline Recommendations): a report of the American College of Cardiology Foundation/ American Heart Association Task Force on Practice Guidelines. J Am Coll Cardiol. 2013 Apr 9;61(14):1555-70.

23. Hirsch AT, Haskal ZJ, Hertzer NR, Bakal CW, Creager MA, Halperin JL, et al. ACC/AHA Guidelines for the Management of Patients with Peripheral Arterial Disease (lower extremity, renal, mesenteric, and abdominal aortic): a collaborative report from the American Associations for Vascular Surgery/Society for Vascular Surgery, Society for Cardiovascular Angiography and Interventions, Society for Vascular Medicine and Biology, Society of Interventional Radiology, and the ACC/AHA Task Force on Practice Guidelines (writing committee to develop guidelines for the management of patients with peripheral arterial disease)--summary of recommendations. J Vasc Interv Radiol JVIR. 2006 Sep;17(9):1383-97; quiz 1398.

24. Fontaine R, Kim M, Kieny R. [Surgical treatment of peripheral circulation disorders]. Helv Chir Acta. 1954 Dec;21(5-6):499-533.

25. Rutherford RB, Baker JD, Ernst C, Johnston KW, Porter JM, Ahn S, et al. Recommended standards for reports dealing with lower extremity ischemia: revised version. J Vasc Surg. 1997 
Sep;26(3):517-38.

26. Rutherford RB, Flanigan DP, Gupta SK. Suggested standards for reports dealing with lower extremity ischemia. J Vasc Surg. 1987 Mar;5(3):501-3.

27. Dormandy JA, Rutherford RB. Management of peripheral arterial disease (PAD). TASC Working Group. TransAtlantic Inter-Society Consensus (TASC). J Vasc Surg. 2000 Jan;31(1 Pt 2):S1-296.

28. Hans SS, DeSantis D, Siddiqui R, Khoury M. Results of endovascular therapy and aortobifemoral grafting for Transatlantic Inter-Society type C and D aortoiliac occlusive disease. Surgery. 2008 Oct;144(4):583-9; discussion 589-590.

29. Aihara H, Soga Y, Mii S, Okazaki J, Yamaoka T, Kamoi D, et al. Comparison of long-term outcome after endovascular therapy versus bypass surgery in claudication patients with TransAtlantic Inter-Society Consensus-II C and D femoropopliteal disease. Circ J Off J Jpn Circ Soc. 2014;78(2):457-64.

30. Baril DT, Chaer RA, Rhee RY, Makaroun MS, Marone LK. Endovascular interventions for TASC II D femoropopliteal lesions. J Vasc Surg. 2010 Jun;51(6):1406-12.

31. Berger JS, Krantz MJ, Kittelson JM, Hiatt WR. Aspirin for the prevention of cardiovascular events in patients with peripheral artery disease: a meta-analysis of randomized trials. JAMA. 2009 May 13;301(18):1909-19.

32. CAPRIE Steering Committee. A randomised, blinded, trial of clopidogrel versus aspirin in patients at risk of ischaemic events (CAPRIE). CAPRIE Steering Committee. Lancet Lond Engl. 1996 Nov 16;348(9038):1329-39.

33. Ramos R, García-Gil M, Comas-Cufí M, Quesada M, Marrugat J, Elosua R, et al. Statins for Prevention of Cardiovascular Events in a Low-Risk Population With Low Ankle Brachial Index. J Am Coll Cardiol. 2016 Feb 16;67(6):630-40.

34. Kumbhani DJ, Steg PG, Cannon CP, Eagle KA, Smith SC, Goto S, et al. Statin therapy and long-term adverse limb outcomes in patients with peripheral artery disease: insights from the REACH registry. Eur Heart J. 2014 Nov 1;35(41):2864-72.

35. Saratzis A, Paraskevopoulos I, Patel S, Donati T, Biasi L, Diamantopoulos A, et al. Supervised Exercise Therapy and Revascularization for Intermittent Claudication: Network Meta-Analysis of Randomized Controlled Trials. JACC Cardiovasc Interv. 2019 Jun 24;12(12):1125-36.

36. Klaphake S, Buettner S, Ultee KH, van Rijn MJ, Hoeks SE, Verhagen HJ. Combination of endovascular revascularization and supervised exercise therapy for intermittent claudication: a systematic review and meta-analysis. J Cardiovasc Surg (Torino). 2018 Apr;59(2):150-7.

37. van den Houten MML, Hageman D, Gommans LNM, Kleijnen J, Scheltinga MRM, Teijink JAW. The Effect of Supervised Exercise, Home Based Exercise and Endovascular Revascularisation on Physical Activity in Patients With Intermittent Claudication: A Network Meta-analysis. Eur J Vasc Endovasc Surg OffJ Eur Soc Vasc Surg. 2019 Sep;58(3):383-92.

38. Layden J, Michaels J, Bermingham S, Higgins B, Guideline Development Group. Diagnosis and management of lower limb peripheral arterial disease: summary of NICE guidance. $B M J$. 2012 Aug 8;345:e4947. 
39. Gerhard-Herman MD, Gornik HL, Barrett C, Barshes NR, Corriere MA, Drachman DE, et al. 2016 AHA/ACC Guideline on the Management of Patients With Lower Extremity Peripheral Artery Disease: A Report of the American College of Cardiology/American Heart Association Task Force on Clinical Practice Guidelines. J Am Coll Cardiol. 2017 21;69(11):e71-126.

40. Harwood A-E, Smith GE, Cayton T, Broadbent E, Chetter IC. A Systematic Review of the Uptake and Adherence Rates to Supervised Exercise Programs in Patients with Intermittent Claudication. Ann Vasc Surg. 2016 Jul;34:280-9.

41. Fanari Z, Weintraub WS. Cost-effectiveness of medical, endovascular and surgical management of peripheral vascular disease. Cardiovasc Revascularization Med Mol Interv. 2015 Nov; 16(7):421-5.

42. Society for Vascular Surgery Lower Extremity Guidelines Writing Group, Conte MS, Pomposelli FB, Clair DG, Geraghty PJ, McKinsey JF, et al. Society for Vascular Surgery practice guidelines for atherosclerotic occlusive disease of the lower extremities: management of asymptomatic disease and claudication. J Vasc Surg. 2015 Mar;61(3 Suppl):2S-41S.

43. TASC Steering Committee, Jaff MR, White CJ, Hiatt WR, Fowkes GR, Dormandy J, et al. An Update on Methods for Revascularization and Expansion of the TASC Lesion Classification to Include Below-the-Knee Arteries: A Supplement to the Inter-Society Consensus for the Management of Peripheral Arterial Disease (TASC II). Vasc Med Lond Engl. 2015 Oct;20(5):465-78.

44. Segal E, Weinberg I, Leichter I, Klimov A, Giri J, Bloom AI. Patient radiation exposure during percutaneous endovascular revascularization of the lower extremity. J Vasc Surg. 2013 Dec;58(6):1556-62.

45. Hirshfeld JW, Ferrari VA, Bengel FM, Bergersen L, Chambers CE, Einstein AJ, et al. 2018 ACC/HRS/NASCI/SCAI/SCCT Expert Consensus Document on Optimal Use of Ionizing Radiation in Cardiovascular Imaging: Best Practices for Safety and Effectiveness: A Report of the American College of Cardiology Task Force on Expert Consensus Decision Pathways. $J$ Am Coll Cardiol. 2018 19;71(24):e283-351.

46. Goldsweig AM, Kennedy KF, Abbott JD, Jones WS, Velagapudi P, Rutar FJ, et al. Patient Radiation Dosage During Lower Extremity Endovascular Intervention. JACC Cardiovasc Interv. 2019 11;12(5):473-80.

47. Chambers CE, Fetterly KA, Holzer R, Lin P-JP, Blankenship JC, Balter S, et al. Radiation safety program for the cardiac catheterization laboratory. Catheter Cardiovasc Interv Off J Soc Card Angiogr Interv. 2011 Mar 1;77(4):546-56.

48. Boc $\mathrm{V}$, Boc $\mathrm{A}$, Zdešar U, Blinc A. Patients' radiation doses during percutaneous endovascular procedures in arteries of the lower limbs. VASA Z Gefasskrankheiten. 2019 Mar;48(2):167-74.

49. Majewska N, Blaszak MA, Juszkat R, Frankiewicz M, Makalowski M, Majewski W. Patients' radiation doses during the implantation of stents in carotid, renal, iliac, femoral and popliteal arteries. Eur J Vasc Endovasc Surg Off J Eur Soc Vasc Surg. 2011 Mar;41(3):372-7.

50. Topaltzikis T, Rountas C, Moisidou R, Fezoulidis I, Kappas C, Theodorou K. Radiation dose to patients and staff during angiography of the lower limbs. Derivation of local dose reference 
levels. Phys Medica PM Int J Devoted Appl Phys Med Biol Off J Ital Assoc Biomed Phys AIFB. 2009 Mar;25(1):25-30.

51. Ketteler ER, Brown KR. Radiation exposure in endovascular procedures. J Vasc Surg. 2011 Jan;53(1 Suppl):35S-38S.

52. Nijssen EC, Rennenberg RJ, Nelemans PJ, Essers BA, Janssen MM, Vermeeren MA, et al. Prophylactic hydration to protect renal function from intravascular iodinated contrast material in patients at high risk of contrast-induced nephropathy (AMACING): a prospective, randomised, phase 3, controlled, open-label, non-inferiority trial. The Lancet. 2017 Apr;389(10076):1312-22.

53. LaBounty TM, Shah M, Raman SV, Lin FY, Berman DS, Min JK. Within-hospital and 30-day outcomes in 107,994 patients undergoing invasive coronary angiography with different lowosmolar iodinated contrast media. Am J Cardiol. 2012 Jun 1;109(11):1594-9.

54. James MT, Samuel SM, Manning MA, Tonelli M, Ghali WA, Faris P, et al. Contrast-induced acute kidney injury and risk of adverse clinical outcomes after coronary angiography: a systematic review and meta-analysis. Circ Cardiovasc Interv. 2013 Feb;6(1):37-43.

55. Grossman PM, Ali SS, Aronow HD, Boros M, Nypaver TJ, Schreiber TL, et al. Contrastinduced nephropathy in patients undergoing endovascular peripheral vascular intervention: Incidence, risk factors, and outcomes as observed in the Blue Cross Blue Shield of Michigan Cardiovascular Consortium. J Intervent Cardiol. 2017 Jun;30(3):274-80.

56. Al Adas Z, Lodewyk K, Robinson D, Qureshi S, Kabbani LS, Sullivan B, et al. Contrast-induced nephropathy after peripheral vascular intervention: Longterm renal outcome and risk factors for progressive renal dysfunction. J Vasc Surg. 2019;69(3):913-20.

57. Ronco C, Bellomo R, Kellum JA. Acute kidney injury. Lancet Lond Engl. 2019 23;394(10212):1949-64.

58. Ronco C, Bellomo R, Kellum J. Understanding renal functional reserve. Intensive Care Med. 2017;43(6):917-20.

59. Delanaye P, Cavalier E, Pottel H. Serum Creatinine: Not So Simple! Nephron. 2017;136(4):3028.

60. Bennett M, Dent CL, Ma Q, Dastrala S, Grenier F, Workman R, et al. Urine NGAL predicts severity of acute kidney injury after cardiac surgery: a prospective study. Clin J Am Soc Nephrol CJASN. 2008 May;3(3):665-73.

61. Erdbruegger U, Palevsky PM, Sheridan AM. Investigational biomarkers and the evaluation of acute tubular necrosis. Uptodate.com; 2014.

62. Cruz DN, Bagshaw SM, Maisel A, Lewington A, Thadhani R, Chakravarthi R, et al. Use of biomarkers to assess prognosis and guide management of patients with acute kidney injury. Contrib Nephrol. 2013;182:45-64. 

PARт Implications in endovascular treatment strategies 
Loss of kidney function after endovascular treatment of peripheral arterial disease

TA. Sigterman ${ }^{1}$, LJJ. Bolt ${ }^{1}$, AG. Krasznai ${ }^{1}$, MG. Snoeijs ${ }^{1}$,

R. Heijboer', GWH. Schurink ${ }^{2}$, LH. Bowman ${ }^{1}$

2. Maastricht University Medical Centre, Maastricht, the Netherland

Published in: 


\section{Abstract}

Background. Administration of radiocontrast during endovascular procedures for peripheral arterial disease may cause acute kidney injury, which generally recovers with supportive treatment. Long-term effects of endovascular procedures on renal function remain to be investigated.

Method. This retrospective observational cohort study includes all patients who newly presented to the vascular surgery outpatient clinic with rutherford class II or III peripheral arterial disease and who were treated with either supervised exercise therapy or endovascular interventions. Changes in estimated glomerular filtration rates (eGFR) after one year were compared between the two treatment groups. Multivariate regression analysis and propensity score-matched paired analysis was done to correct for potential confounders.

Results. One year after treatment, eGFR was reduced by $8.6 \mathrm{~mL} / \mathrm{min}(95 \% \mathrm{CI}: 7.3$ - 9.9, $\mathrm{P}<0.001)$ after endovascular intervention $(\mathrm{N}=284)$ and by $1.7 \mathrm{~mL} / \mathrm{min}(95 \% \mathrm{CI}: 0.9-$ $25, \mathrm{P}<0.001)$ after supervised exercise therapy $(\mathrm{N}=299)$. After correction for potential confounders, endovascular interventions were associated with $9.2 \mathrm{~mL} / \mathrm{min}$ (95\%CI: 5.9 - 12.4, $\mathrm{P}<0.001)$ more renal decline as compared to exercise therapy. Similar results were found in the propensity score-matched paired analysis.

Conclusion. Endovascular procedures for peripheral arterial disease are associated with clinically relevant and long-term loss of kidney function. 


\section{Introduction}

Peripheral arterial disease (PAD) is a common disease with an increasing prevalence. Patients may present with intermittent claudication and rest pain or tissue loss due to critical limb ischemia. Intermittent claudication may be treated with supervised exercise therapy (SET) or with endovascular interventions. Although long-term effects on walking distance and quality of life of these treatment modalities are similar, endovascular interventions result in instant restoration of blood flow and faster resolution of symptoms. ${ }^{1}$ However, these invasive procedures are associated with complications including contrast-induced nephropathy.

The past decade has witnessed increasing awareness of acute kidney injury (AKI) after various studies have demonstrated that relatively small increases in serum creatinine concentration are associated with increased in-hospital mortality. ${ }^{2}$ Acute kidney injury may be caused by a combination of ischemia, sepsis and administration of nephrotoxic agents including radiocontrast. Although renal function generally recovers with supportive therapy, patients with acute kidney injury remain at increased risk of long- term renal insufficiency. ${ }^{3}$ In particular, patients with fast decline of glomerular filtration rate $(>4 \mathrm{~mL} /$ $\mathrm{min} /$ year) are at increased risk of cardiovascular disease and death. ${ }^{4}$

Although the acute effects of radiocontrast administration on renal function have been well studied, long-term effects of endovascular interventions for peripheral arterial disease remain to be investigated. Therefore, we studied whether endovascular treatment of intermittent claudication is associated with long-term loss of renal function and which individuals are at increased risk of adverse renal outcomes after radiocontrast administration.

\section{Methods}

\section{Study design}

This retrospective observational cohort study was conducted at a large hospital in the Netherlands. In the period from January $1^{\text {st }} 2011$ until July $31^{\text {st }} 2013$, we included all new patients with intermittent claudication Rutherford classification II-III who were 
treated with endovascular interventions or supervised exercise therapy. Rutherford class II was defined as moderate intermittent claudication with a walking distance between 200-500 meters. Rutherford class III was defined as severe intermittent claudication, walking distance less than 200 meters. Patients who had previously been treated with endovascular interventions (including percutaneous coronary interventions) or who received an endovascular intervention or diagnostic study using radiocontrast within the first year after the index treatment were excluded from analysis. Additionally, patients with Rutherford IV-VI, acute ischemia, no baseline renal function test or end- stage renal disease were excluded from the study. Glomerular filtration rates were estimated according to the CKD-EPI formula. The lowest and shortest serum creatinine concentration in time from the intervention but within a maximum period of six months pre-procedural was used to calculate baseline eGFR, and the lowest serum creatinine concentration measured between 11 and 13 months after the index treatment was used to calculate eGFR after 1 year.

Patients without baseline renal function measurements or with end-stage renal disease at baseline were excluded from the analysis. Loss of renal function in the first year after treatment for intermittent claudication was estimated and compared between patients receiving endovascular interventions and supervised exercise therapy. Fast renal decline was defined as more than $4 \mathrm{~mL} / \mathrm{min}$ loss of eGFR in one year. The study was carried out in accordance with the declaration of Helsinki and was approved by the institutional review board.

\section{Treatment selection}

In all patients, ankle-brachial indices were measured and aorta-iliac duplex scanning was performed. All patients were treated according to the national guideline for the treatment of peripheral arterial disease. In case of significant aorta-iliac stenosis of $>70 \%$, MR angiography was ordered and patients were evaluated for endovascular intervention by a multidisciplinary team of vascular surgeons and interventional radiologists. Patients without significant aorta-iliac stenosis were primarily treated with supervised exercise therapy. All patients were prescribed statins and anti-platelet drugs. 


\section{Endovascular interventions}

All endovascular procedures were performed by two experienced staff interventional radiologist and/or in association with one of three vascular surgeons. All procedures were executed in an endovascular suite equipped with a Philips Allure Xper FD20 (Philips, Eindhoven, the Netherlands) on a carbon fibre movable interventional table. Pulse beam radiation was routinely utilized. Two types of contrast agents were utilized: Xenetix 300 (Geurbet, Gorinchem, the Netherlands) in patients requiring hydration or with known allergies to radiocontrast, whereas all other patients received Omnipaque 240 (GE Healthcare, Pittsburgh, PA). In our hospital we utilize a national renal protective program, which includes the following; Nephrotoxic drugs (NSAIDs, diuretics, ACE- inhibitors, metformin, digoxin, sotalol, lithium and colchicine) were not taken on the day of the intervention. In concurrence with our guideline none of the patients were advised to stop angiotensin receptor blocker medication.

Patients with eGFR $<45 \mathrm{~mL} / \mathrm{min}$ or with eGFR $<60 \mathrm{~mL} / \mathrm{min}$ and additional risk factors for contrast-induced nephropathy (diabetes mellitus, heart failure, age $>75$ years, hypotension, and anaemia) received pre-procedural hydration. Standard hydration regime consists of four hours of hydration with sodium-bicarbonate and high-risk cardiac patients received twelve hours of hydration with sodium-bicarbonate. All patients were routinely seen after four weeks for evaluation of symptoms. Patients were not referred for supervised exercise therapy within the first year after endovascular intervention.

\section{Supervised exercise therapy}

Supervised exercise therapy was offered by a local network of physiotherapists and consisted of intensive standardized treadmill exercises for three months. Patients were seen three times a week in the first month, and two times a week in the second and third months. General muscular strengthening or arm exercises were not prescribed. All patients were routinely seen after three months for evaluation of symptoms. Patients who received additional endovascular intervention within the first year of exercise therapy were excluded from the study. 


\section{Statistical analyses}

Continuous variables were expressed as means with standard deviations (SD) and categorical variables were expressed as percentages. Missing data were replaced with the mean of five imputations. Baseline characteristics and outcomes of the two study groups were compared with Student t-tests for continuous variables and with chi-square tests for categorical variables. To account for potential confounders, loss of kidney function between the two study groups was compared with multivariable linear regression analysis including baseline characteristics. Fast renal decline in the two study groups was compared with multivariable logistic regression analysis using the Wald test for statistical significance. Clinically relevant interaction terms were added to the regression models and retained in case of statistical significance, respectively (Age x GFR / AGE x DM / Age x renal decline / Age $\mathrm{x}$ ABI). Assumptions of the regression models were checked with normal probabilityprobability, residual plots and by addition of quadratic terms when appropriate; these terms were retained in the model if statistically significant. Furthermore, multicollinearity and influential outliers were checked using variance inflation factors and Cook's distance.

To further reduce the effect of potential confounding, loss of kidney function was analyzed using propensity-matched paired analysis. The propensity of receiving an endovascular intervention rather than supervised exercise therapy was estimated using multivariable logistic regression. Patients treated with endovascular procedures were matched 1:1 with patients treated with supervised exercise therapy without replacement based on a maximal difference within pairs of 0.2 standard deviations of the logit propensity score. ${ }^{6}$ In the matched pairs, continuous variables were compared with paired Student t-tests and categorical variables were compared with McNemar tests. Results with P less than 0.05 were considered statistically significant. All statistical computing was carried out by T.A.S. and M.G.S. using SPSS version 21 (SPSS Inc., Chicago, IL).

Sample sizes were estimated assuming a mean difference in loss of kidney function of $3 \mathrm{~mL} / \mathrm{min}$ between the two study groups and a standard deviation of $8 \mathrm{~mL} / \mathrm{min}$ with $\alpha=0.05$ and $\beta=0.20$. With these assumptions, some 224 patients were required in each study group. 


\section{Results}

We studied all patients with intermittent claudication due to peripheral arterial disease who newly presented at the vascular surgery outpatient clinical over a 2.5 -year period (Figure 1). In total 299 patients were prescribed supervised exercise therapy and 284 patients were treated with endovascular procedures (Table 1). One-year post-procedural eGFR results were available for 411 patients. Patients receiving endovascular interventions were significantly younger $(64.5$ vs. 67.9 years, $\mathrm{P}<0.01)$ and had significantly better baseline renal function $(74.8$ vs. $69.8 \mathrm{~mL} / \mathrm{min}, \mathrm{P}<0.01)$ as compared to patients receiving supervised exercise therapy.

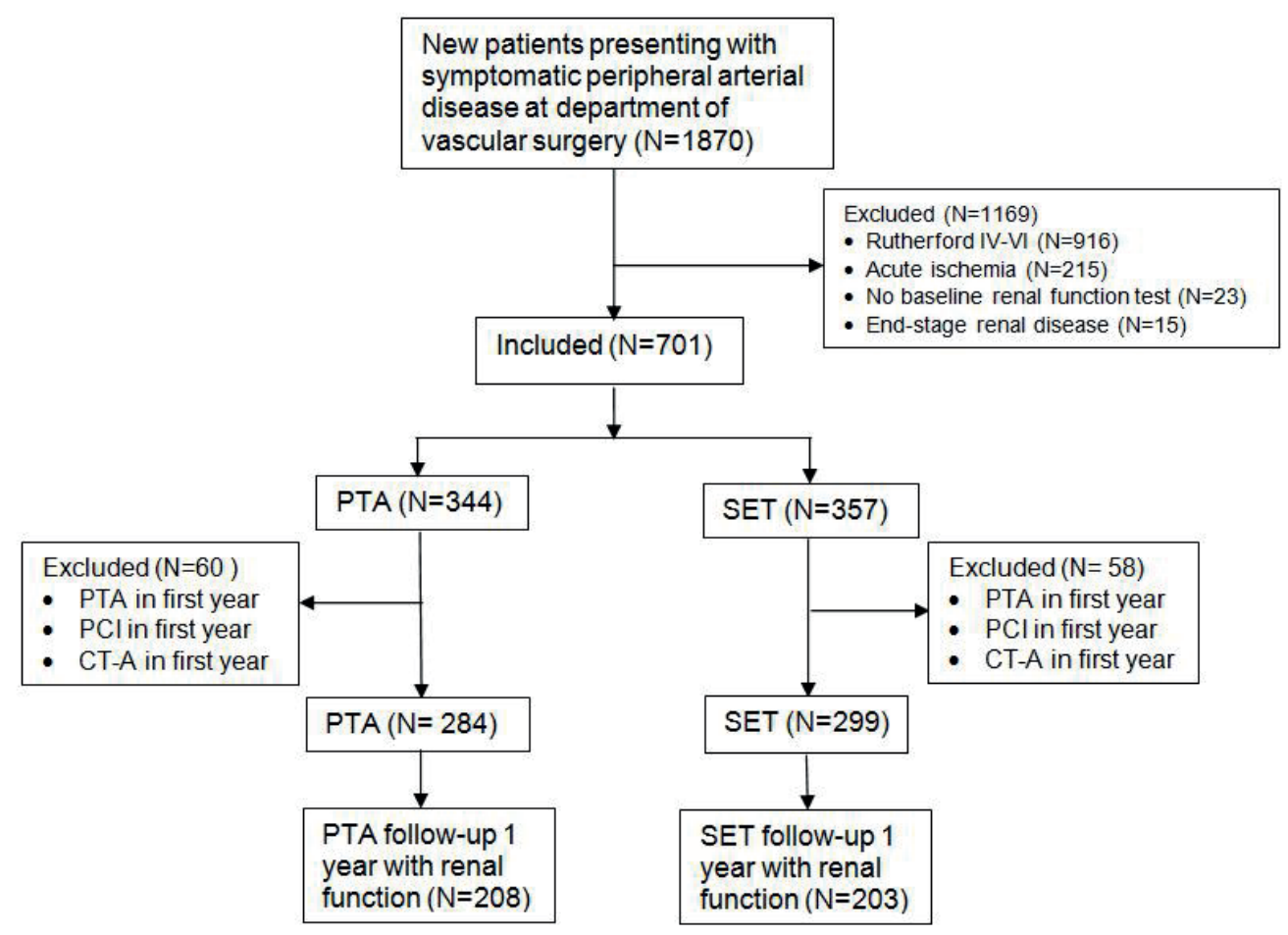

Figure 1: Flowchart of patient enrolment

Furthermore, patients treated with endovascular procedures were more often smokers and had less often diabetes mellitus $(\mathrm{P}<0.01)$. In contrary, smoking was not significantly different between the two study groups one-year post-procedural $(\mathrm{P}=0.61)$. The primary anatomic location of the stenotic lesion in the endovascular study population was in the 
pelvic region, respectively $243(86 \%)$, compared to non-invasive group were the main anatomic location was femoral $137(46 \%)$, followed by multi-level $(\mathrm{P}<0.01)$. Stent placement was performed in 200 patients $(70.4 \%)$ in the endovascular group.

Table 1: Baseline characteristics (Imputed file)

\begin{tabular}{|c|c|c|c|}
\hline & $\begin{array}{l}\text { Endovascular } \\
\text { intervention } \\
(\mathrm{N}=\mathbf{2 8 4})\end{array}$ & $\begin{array}{c}\text { Supervised exercise } \\
\text { therapy } \\
(\mathrm{N}=\mathbf{2 9 9})\end{array}$ & $\mathbf{P}$ \\
\hline Age (yrs.) & $65(37-87)$ & $68(31-90)$ & $<0.01$ \\
\hline Sex (male) & $65 \%$ & $63 \%$ & 0.70 \\
\hline $\mathrm{BMI}\left(\mathrm{kg} / \mathrm{m}^{2}\right)$ & $27(14-52)$ & $28(16-59)$ & 0.02 \\
\hline Rutherford classification & & & $<0.01$ \\
\hline 2 & ०\% & $64 \%$ & \\
\hline 3 & $100 \%$ & $36 \%$ & \\
\hline Symptom duration (months) & $15(2-96)$ & $14(2-72)$ & 0.23 \\
\hline Ankle-brachial index & $0.73\left(0.3^{6-1.26)}\right.$ & $0.73(0.22-1.29)$ & 0.95 \\
\hline Baseline eGFR (mL/min) & $75(16-90)$ & $70(19-90)$ & $<0.01$ \\
\hline$>60 \mathrm{~mL} / \mathrm{min}$ & $82 \%$ & $71 \%$ & \\
\hline $30-60 \mathrm{~mL} / \mathrm{min}$ & $17 \%$ & $26 \%$ & \\
\hline $15-30 \mathrm{~mL} / \mathrm{min}$ & $1 \%$ & $2 \%$ & \\
\hline$<15 \mathrm{~mL} / \mathrm{min}$ & $0 \%$ & \%\% & \\
\hline Diabetes mellitus (yes) & $15 \%$ & $33 \%$ & $<0.01$ \\
\hline Hypertension (yes) & $62 \%$ & $69 \%$ & 0.08 \\
\hline Smoking (yes) & $62 \%$ & $52 \%$ & 0.01 \\
\hline Hypercholesterolemia (yes) & $55 \%$ & $59 \%$ & 0.30 \\
\hline $\begin{array}{l}\text { Family history of } \\
\text { cardiovascular disease (yes) }\end{array}$ & $46 \%$ & $46 \%$ & 0.99 \\
\hline Heart failure (yes) & $1 \%$ & $2 \%$ & 0.41 \\
\hline Contrast (ml) & $80(20-210)$ & - & $<0.01$ \\
\hline ACE & $42 \%$ & $38 \%$ & 0.61 \\
\hline ARBS & $9 \%$ & $13 \%$ & 0.35 \\
\hline Statin & $88 \%$ & $91 \%$ & 0.82 \\
\hline
\end{tabular}

Data are presented as median (range) or as percentages.

In patients receiving endovascular procedures for intermittent claudication, estimated glomerular filtration rates (eGFR) decreased by $8.6 \pm 10.7 \mathrm{~mL} / \mathrm{min}(\mathrm{P}<0.001)$ in the first year after treatment (Figure 2). 


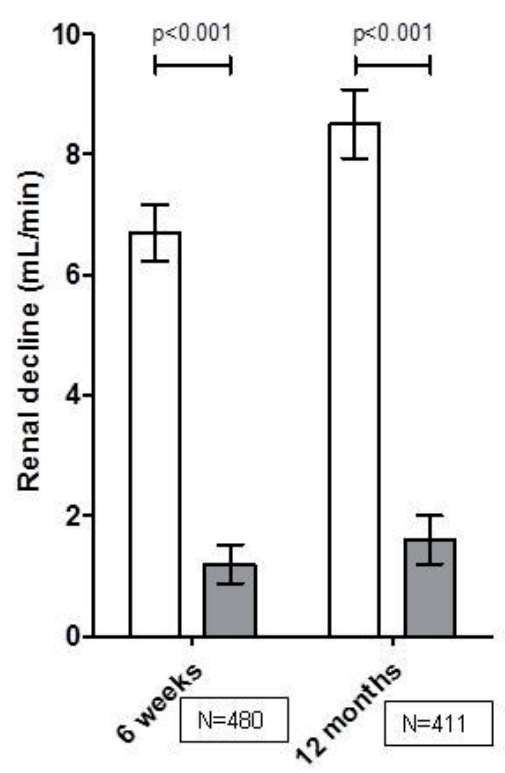

Figure 2: Loss of kidney function after treatment for intermittent claudication

In the same period, renal function decreased by $1.7 \pm 6.5 \mathrm{~mL} / \mathrm{min}(\mathrm{P}<0.001)$ in patients who received supervised exercise therapy. Although eGFR rates decreased in both groups, loss of renal function was significantly greater in patients after endovascular interventions $(\mathrm{P}<0.001)$. Furthermore, $64 \%$ of patients receiving endovascular interventions experienced fast renal decline (defined as $>4 \mathrm{~mL} / \mathrm{min}$ loss of eGFR within one year) as compared to $23 \%$ of patients with supervised exercise therapy $(\mathrm{P}<0.001)$.

Multivariable linear regression was used to correct for potential confounders of the association between endovascular procedures and loss of renal function (Table 2). When clinically relevant patient characteristics were included in the analysis, endovascular intervention was associated with a $9.2 \mathrm{~mL} / \mathrm{min}$ reduction $(95 \% \mathrm{CI}: 5.9-12.4, \mathrm{P}<0.001$ ) of eGFR and with an 11.0-fold (95\%CI: 4.6-26, P<0.001) increased odds of fast renal decline after one year as compared to supervised exercise therapy. The interaction between treatment and baseline renal function was not statistically significant. Sensitivity tests without imputation of missing data and without patients with rutherford II peripheral arterial disease showed similar results. Further correction for potential confounders was 
done with propensity score-matched paired analysis. Some 384 (66\%) patients could be paired and were included in this analysis. Baseline characteristics within the paired analysis were similar between the two treatment groups. Renal function of patients receiving endovascular interventions decreased $9.4 \mathrm{~mL} / \mathrm{min}(95 \% \mathrm{CI}: 7.5-11.0, \mathrm{P}<0.001)$ more than in patients with supervised exercise therapy. Furthermore, $67 \%$ of patients treated with endovascular procedures experienced fast renal decline as compared to $24 \%$ of patients with supervised exercise therapy

Table 2: Multivariate linear regression of loss of kidney function and multivariate logistic regression of fast renal decline

\begin{tabular}{|c|c|c|c|c|c|c|}
\hline & \multicolumn{2}{|c|}{ Renal decline ( $\mathrm{mL} / \mathrm{min})$} & \multicolumn{4}{|c|}{ Fast renal decline (yes) } \\
\hline & $\begin{array}{c}\text { Mean } \\
\text { difference }\end{array}$ & $95 \%(\mathrm{Cl})$ & $\mathbf{P}$ & OR & $95 \%(\mathrm{Cl})$ & $\mathbf{P}$ \\
\hline PTA & -9.15 & $(-12.39-5.90)$ & $<0.01$ & 11.0 & $(4 \cdot 6-25 \cdot 96)$ & $<0.01$ \\
\hline Gender (male) & 0.23 & $(-1.27-1.73)$ & 0.76 & 0.92 & $\left(0.63-1.3^{6}\right)$ & 0.69 \\
\hline Age (/years) & 0.01 & $(-0.07-0.09)$ & 0.81 & 0.98 & $(0.96-0.99)$ & 0.04 \\
\hline $\mathrm{BMI}\left(/ \mathrm{kg} / \mathrm{m}^{2}\right)$ & 0.10 & $(-0.04-0.25)$ & 0.15 & 1.01 & $(0.98-1.05)$ & 0.48 \\
\hline \multicolumn{7}{|l|}{ Rutherford } \\
\hline Classification II(ref) & - & - & - & - & - & - \\
\hline Classification III & -1.03 & $(-3.12-1.05)$ & 0.33 & 1.71 & $(0.93-3.16)$ & 0.08 \\
\hline ABI (pre-operative) & -2.76 & $(-7.17-1.65)$ & 0.22 & 0.30 & $(0.09-0.96)$ & 0.04 \\
\hline Smoking & 0.82 & $(-0.72-2.36)$ & 0.30 & 1.13 & $(0.79-1.64)$ & 0.50 \\
\hline Diabetes Mellitus & 0.30 & $(-1.73-1.79)$ & 0.83 & 1.00 & $(0.63-1.59)$ & 0.99 \\
\hline Heart Failure & -2.83 & $(-8.07-2.41)$ & 0.29 & 6.54 & $(1.55-27.6)$ & 0.01 \\
\hline Hypertension & -2.19 & $(-3.80--0.58)$ & $<0.01$ & 1.95 & $(1.27-3.0)$ & $<0.01$ \\
\hline GFR (pre-operative) & 0.07 & $(0.02-0.12)$ & $<0.01$ & 1.01 & $(0.99-1.0)$ & 0.17 \\
\hline Anatomic location (iliac) & -0.04 & $(-0.97-0.89)$ & 0.93 & 0.59 & $(0.25-1.3)$ & 0.19 \\
\hline Contrast $>150 \mathrm{~mL}$ & -1.48 & $(-7 \cdot 9-4 \cdot 9)$ & 0.65 & 1.63 & $(0.65-4.1)$ & 0.30 \\
\hline Proteinuria (<0.14 gr/24) & 3.48 & $(-1.6-8.5)$ & 0.17 & 1.32 & $(0.68-2.6)$ & 0.41 \\
\hline Systolic BP pre $>140 \mathrm{mmHg}$ & -0.41 & $(-4 \cdot 1-3 \cdot 4)$ & 0.83 & 0.87 & $(0.54-1.41)$ & 0.58 \\
\hline Systolic BP post >140mm Hg & $-4 \cdot 51$ & $(-8.9--0.15)$ & 0.04 & 0.92 & $(0.55-1.53)$ & 0.75 \\
\hline
\end{tabular}

Linear model of predictors of renal decline, with $95 \% \mathrm{Cl}$ interval and P-values. Coefficients of the model predicting fast renal decline, showing odds-ratio (OR), $95 \%$ confidence interval and P-values.

Finally, we studied which variables were associated with fast renal decline after endovascular treatment of peripheral arterial disease (Table 3). Patients with hypertension were at significantly greater risk of fast renal decline (OR 2.3, 95\%CI: $1.3-4.0, \mathrm{P}<0.01)$. 
However, radiocontrast dose and traditional risk factors for chronic kidney disease (CKD) such as age, baseline renal function and diabetes mellitus were not associated with fast renal decline.

Table 3: Sub-analyses PTA group for fast renal decline

\begin{tabular}{lccc}
\hline & \multicolumn{2}{c}{ Fast renal decline (yes) } & \\
\hline Gender (male) & OR & $95 \%(C l)$ & P \\
Age (/years) & 0.90 & $(0.53-1.51)$ & 0.20 \\
BMI $\left(/ \mathrm{kg} / \mathrm{m}^{2}\right)$ & 0.98 & $(0.95-1.01)$ & 0.69 \\
Rutherford & 0.99 & $(0.94-1.04)$ & 0.61 \\
Classification II(ref) & & & \\
Classification III & - & - & 0.94 \\
ABI (pre-operative) & 1.06 & $(0.24-4.72)$ & 0.11 \\
Smoking & 0.29 & $(0.06-1.30)$ & 0.97 \\
Diabetes Mellitus & 1.01 & $(0.58-1.76)$ & 0.84 \\
Heart Failure & 0.93 & $(0.46-1.88)$ & 0.36 \\
Hypertension & 2.90 & $(0.29-29.67)$ & $<0.01$ \\
GFR (pre-operative) & 2.30 & $(1.32-4.00)$ & 0.09 \\
\hline
\end{tabular}

\section{Discussion}

In the current study, we describe the long-term loss of kidney function in patients who received endovascular interventions for intermittent claudication. These patients experience an $8.5 \pm 10.7 \mathrm{~mL} / \mathrm{min}$ reduction of eGFR within the first year after the intervention, which is significantly greater than similar patients who were offered supervised exercise therapy. Furthermore, some $64 \%$ of patients have a reduction in renal function of $>4 \mathrm{~mL} / \mathrm{min}$ in the year following endovascular treatment. This fast renal decline has previously been shown a predictor of increased mortality, cardiovascular events and end-stage renal disease. Therefore, our findings are of clinical importance and efforts should be made to prevent possible progressive loss of kidney function in these patients.

The loss of kidney function after endovascular interventions is probably caused by exposure to nephrotoxic contrast media. Hyperosmolar contrast media are concentrated in the renal medulla and will reduce tubular and capillary flow due to its high viscosity. This increases 
the direct toxic effects of iodinated contrast media on tubular and endothelial cells. The resulting tubular congestion and medullary vasoconstriction will cause an acute reduction in glomerular filtration rate and tissue oxygen concentration. Reducing the medullary concentration of contrast media by generous hydration may prevent contrast-induced acute kidney injury. ${ }^{6}$

Contrast-induced nephropathy generally recovers with supportive treatment. However, patients with acute kidney injury, even after initial full recovery of renal function, remain at increased risk of end-stage renal disease. Animal experiments have shown that renal ischemia and reperfusion causes permanent loss of peritubular capillaries that will result in progressive fibrosis and loss of kidney function. ${ }^{7}$ Histological analysis of renal transplant biopsies suggests that similar mechanisms play a role in clinical medicine. ${ }^{7}$ We suggest that similar mechanisms may explain the long-term loss of kidney function after endovascular intervention for intermittent claudication.

In contrast to the traditional paradigm, loss of kidney function is not necessarily constant over time. Many patients with chronic kidney disease have non-linear trajectories of renal decline with prolonged periods of stable kidney function interspersed by episodes of rapid loss of renal function. ${ }^{8}$ Our study suggests that exposure to contrast media during endovascular interventions for intermittent claudication may elicit some of these episodes. In line with our findings, it has previously been shown that patients who experience acute kidney injury after coronary angiography have faster rates of renal decline than patients without acute kidney injury. ${ }^{9}$ Furthermore, the cumulative exposure to contrast media is independently associated with progression to severe chronic kidney disease in patients with peripheral arterial disease. ${ }^{10}$ To understand which patients are at risk of rapid loss of kidney function, we determined which baseline characteristics were associated with fast renal decline after endovascular procedures for intermittent claudication. Traditional risk factors for progressive chronic kidney disease such as age, baseline renal function and diabetes mellitus were not associated with the rate of renal function decline in the first year after endovascular interventions. This indicates that further research is necessary to unravel the pathophysiology of long-term loss of renal function after contrast-induced acute kidney injury and to identify patient subsets at increased risk of permanent loss of renal function after endovascular interventions for intermittent claudication.

Several other factors that could possible cause renal decline should be mentioned, respectively micro-embolization into the renal arteries, due to catheter manipulation 
during endovascular procedures and finally the MR-angiography. Reported incidence of micro-embolization following coronary angiography are $0.9 \%$. The incidence of acute kidney injury following trans-radial approach is significantly lower than for the transfemoral approach in coronary interventions. ${ }^{11}$

Additionally, the risk of nephrogenic systemic fibrosis (NSF) after exposure to Gadolinium during MR-angiography should be mentioned. NSF is a rare condition appearing only in patients with severe renal impairment or failure. This risk however, is negligible when the set limit is not exceeded and since the establishment of guidelines and the increased general awareness of this condition, the occurrence of NSF has been nearly eliminated. Lastly, significantly more patients with Rutherford classification III were treated with invasively, which possible might be the reason why this study population was more prone to a decline in renal function. However, ankle-brachial index is similar and the definition of Rutherford classification is subjective and subject to interpretation between physicians. So, it is the authors' opinion that there is a significant difference in disease staging, however this is not seen in the objective ankle-brachial index and should therefore not be the reason for the major difference in renal decline. Finally, as stated by Bunte et al, even in patients with critical limb ischemia the ABI may be normal or mildly reduced, which may be explained by arterial stiffness due to diabetes mellitus. Subsequently, in this case ABI is not necessarily an accurate reflection of disease severity. ${ }^{12}$

In this retrospective observational cohort study, we observed a large and clinically relevant reduction in kidney function after endovascular procedures for intermittent claudication. This loss of kidney function was greater than the natural reduction in glomerular filtration rate both in the general population and in a cohort of patients with intermittent claudication who were treated with supervised exercise therapy. The Kidney Disease Outcomes Quality Initiative guidelines developed by the National Kidney Foundation state that eGFR should normally decrease by $1 \mathrm{~mL} \cdot \mathrm{min}^{-1} \cdot 1.73 \mathrm{~m}^{-2}$ per year once adulthood is reached. ${ }^{13,14}$ In addition, several studies show that rapid decline in eGFR is associated with an increased risk of cardiovascular and all-cause mortality in older adults, independent of baseline eGFR and other demographic variables. ${ }^{15-17}$ 


\section{Limitations}

Although these patients presented to our outpatient clinic in the same time period, individuals treated with endovascular interventions differed in several ways from patients who received supervised exercise therapy. These differences in baseline characteristics between the two study groups may confound the interpretation of our findings. Nevertheless, correction for possible confounders by multivariable regression analysis and by creating pairs of patients with the same chance of receiving either one of the treatment modalities did not change our findings. Since we corrected for the most important risk factors for acute and chronic kidney disease, we consider it highly unlikely that unmeasured confounders - presumably with less impact on the loss of kidney function - would change the conclusions of our study. Obviously, only a randomized clinical trial comparing endovascular interventions with supervised exercise therapy would entirely eliminate selection bias. Moreover, supervised exercise therapy may have been protective in renal decline, it is therefore our opinion that this randomized controlled trial should also include patients treated with endovascular intervention followed by supervised exercise therapy.

Unfortunately, serum creatinine was not routinely measured in the first days after the endovascular interventions and we are therefore unable to provide information on the incidence and impact of contrast-induced acute kidney injury in our cohort. Furthermore, the follow-up period was too short to determine the effect of the loss of kidney function on long-term mortality, cardiovascular events, and end-stage renal disease. We are currently working on a prospective observational cohort study to address these outstanding issues. Finally, in $28 \%$ of patients renal function was not measured at one year after treatment, which may have led to a selection bias. Nevertheless, this rather large proportion of missing data did not seem to invalidate our findings, since analysis with and without imputation of missing values produced similar results. However, our sample size was based on 224 patients in each group, due to the lost-to follow-up we had only data of 203 patients in one study group. 


\section{Conclusion}

Endovascular procedures for intermittent claudication are associated with clinically relevant and long-term loss of kidney function. With the increasing availability and complexity of endovascular interventions for patients with peripheral arterial disease, these findings indicate that kidney function should be closely monitored in these patients to initiate timely treatment and prevent possible progressive chronic kidney disease. 


\section{References}

1. Ahimastos AA, Pappas EP, Buttner PG, Walker PJ, Kingwell BA, Golledge J. A meta-analysis of the outcome of endovascular and non-invasive therapies in the treatment of intermittent claudication. J Vasc Surg 2011;54(5):1511-21

2. Weisbord SD, Chen H, Stone RA, Kip KE, Fine MJ, Saul MI, et al. Association of increases in serum creatinine with mortality and length of hospital stay after coronary angiography. $J \mathrm{Am}$ Soc Nephrol 2006;17(10):2871-7

3. Waikar SS, Winkelmayer WC. Chronic on Acute Renal Failure Long-term Implications of Severe Acute Kidney Injury. JAMA 2009;302(11):1227-1229

4. Henry RM, Kostense PJ, Bos G, Dekker JM, Nijpels G, Heine RJ, et al. Mild renal insufficiency is associated with increased cardiovascular mortality: the Hoorn Study. Kidney International 2002;62:1402-1407

5. Austin PC, Statistical criteria for selecting the optimal number of untreated subjects matched to each treated subject when using many-to-one matching on the propensity score. Am J Epidemiol 2010;172(9):1092-7

6. Seeliger E, Sendeski M, Rihal CS, Persson PB. Contrast-induced kidney injury: mechanisms, risk factors, and prevention. Eur Heart J 2012;33(16):2007-15

7. Levey AS, Coresh J, Greene T, Marsh J, Stevens LA, Kusek JW, et al. Expressing the Modification of Diet in Renal Disease Study equation for estimating glomerular filtration rate with standardized serum creatinine values. Clin Chem 2007;53(4):766-772

8. Li L, Astor BC, Lewis J, Hu B, Appel LJ, Lipkowitz MS, et al.

9. Longitudinal progression trajectory of GFR among patients with CKD. Am J Kidney Disease 2012;59(4):504-512

10. James MT, Ghali WA, Tonelli M, Faris P, Knudtson ML, Pannu N, et al. Acute kidney injury following coronary angiography is associated with a long-term decline in kidney function. Kidney Int 2010;78(8):803-809

11. Kougias P, Sharath S, Barshes NR, Lowery B, Garcia A, Pak T, et al. Impact of cumulative intravascular contrast exposure on renal function in patients with occlusive and aneurysmal vascular disease. J Vasc Surg 2014;59(6):1644-50

12. Cortese B, Sciahbasi A, Sebik R, Rigattieri S, Alonzo A, Silva-Orrego P, et al. Comparison of risk of acute kidney injury after primary percutaneous coronary interventions with the transradial approach versus the transfemoral approach (from the PRIPITENA urban registry). Am J Cardiol. 2014 Sep 15;114(6):820-5

13. Bunte MC, Jacob J, Nudelman B, Shishehbor MH. Validation of the relationship between ankle-brachial and toe-brachial indices and infragenicular arterial patency in critical limb ischemia. Vasc Med. 2015 Feb;20(1):23-9

14. Vickery S, Stevens PE, Dalton RN, van Lente F, Lamb EJ. Does the ID-MS traceable MDRD equation work and is it suitable for use with compensated Jaffé and enzymatic creatinine 
assays? Nephrology Dialysis Transplantation. 2006;21(9):2439-2445. / 22

15. Chen S-C, Chang J-M, Liu W-C, Tsai YC, Tsai JC, Hsu PC, et al. Brachial-ankle pulse wave velocity and rate of renal function decline and mortality in chronic kidney disease. Clinical Journal of the American Society of Nephrology. 2011;6(4):724-732)

16. Rifkin DE, Shlipak MG, Katz R, Phil D, Fried LF, Siscovick D, et al. Rapid Kidney Function Decline and Mortality Risk in Older Adults Arch Intern Med. 2008 Nov 10; 168(20): 22122218

17. Shlipak MG, Katz R, Kestenbaum B, Siscovick D, Fried L, Newman A et al. Rapid decline of kidney function increases cardiovascular risk in the elderly. J. Am. Soc. Nephrol. 20,26252630 (2009)

18. Al-Aly Z. Zeringue A, Fu J, Rauchman MI, McDonald JR, El-Achkar TM Rate of Kidney Function Decline Associates with Mortality. J Am Soc Nephrol 21: 1961-1969, 2010. 
Loss of kidney function in patients with critical limb ischemia treated endovascular or surgically

Tim A Sigterman ${ }^{1}$, Lars JJ Bolt ${ }^{1}$, Attila G Krasznai ${ }^{1}$, Marten G Snoeijs ${ }^{1}$,

Rel Heijboer ${ }^{1}$, Geert-Willem H. Schurink ${ }^{2}$, LH. Bowman ${ }^{1}$

1. Zuyderland Medical Centre, Heerlen, the Netherlands
University Medical Centre, Maastricht, the Netherlands

Published in: 


\section{Abstract}

Background. Acute kidney injury following contrast administration during endovascular procedures for peripheral arterial disease generally recovers with supportive treatment. However, long-term effects of endovascular procedures for critical limb ischemia on renal function remain to be investigated.

Method. This retrospective observational cohort study includes all patients who newly presented to the vascular surgery outpatient clinic with rutherford class IV-VI peripheral arterial disease and who were treated with either endovascular or surgical interventions. Changes in estimated glomerular filtration rates (eGFR) after one year were compared between the two types of intervention. Multivariate linear regression analysis was done to correct for potential confounders.

Results. One year after treatment, eGFR was reduced by $15.0 \mathrm{~mL} / \mathrm{min}$ (95\%CI: 13.1 17.0, $\mathrm{P}<0.001)$ after endovascular interventions $(\mathrm{N}=209)$ and by $7.6 \mathrm{~mL} / \mathrm{min}(95 \% \mathrm{CI}$ : $5.1-10.0, \mathrm{P}<0.001)$ after surgical therapy $(\mathrm{N}=81)$. Although eGFR rates decreased in both groups, loss of renal function was significantly greater in patients after endovascular interventions $(\mathrm{P}<0.001)$. Furthermore, $77 \%$ of patients receiving endovascular interventions experienced fast renal decline (defined as $>4 \mathrm{~mL} / \mathrm{min}$ loss of eGFR within one year) as compared to $54 \%$ of patients treated surgically $(\mathrm{P}<0.001)$. After correction for potential confounders, endovascular intervention was associated with $7.4 \mathrm{~mL} / \mathrm{min}$ (95\%CI: 5.4-9.3, $\mathrm{P}<0.001)$ greater loss of renal function compared to patients treated surgically.

Conclusion. Endovascular procedures for critical limb ischemia are associated with clinically relevant permanent long-term loss of kidney function. This loss of renal function is greater than in comparable patients who were treated with open surgery. 


\section{Introduction}

Critical limb ischemia (CLI), the most severe manifestation of peripheral arterial disease (PAD), with patients presenting with ischemic rest pain, ulcerations, or both. Epidemiologic data predict a burgeoning epidemic of critical limb ischemia, due to the increasing incidence and prevalence of atherosclerotic risk factors, especially age, diabetes and chronic kidney disease (CKD). With the fast progression of endovascular technology in the last decades, a shift from surgical bypass to endovascular therapy as a first-line intervention for patients with critical limb ischemia has occurred. However, current available evidence suggests that there is no difference in clinical outcomes for patients with CLI treated endovascular or with surgical intervention.

The authors have witnessed increasing awareness of acute kidney injury (AKI) after several studies have demonstrated that relatively small increases in serum creatinine concentration are associated with increased development of chronic kidney disease. ${ }^{1}$ According to the KDOQI guideline, acute kidney injury is defined as $>50 \%$ increase in serum creatinine within 48hours post-procedural. ${ }^{2}$ Moreover, reports show that acute kidney injury is associated with higher occurrence of long-term morbidity such as progression to chronic kidney disease and development of end-stage renal disease (ESRD). ${ }^{3,4}$ In addition, longterm survival in patients with acute kidney injury is significantly decreased. ${ }^{5,6}$

Although the acute effects of radiocontrast administration on renal function have been well studied. Limited data is available about the impact of contrast material on renal function during long-term follow-up in patients with critical limb ischemia treated with endovascular interventions. Merely one study, predominantly males (99\%), studied the effect of acute kidney injury following endovascular or surgical revascularization in patient with critical limb ischemia, showing that acute kidney injury is associated with the development of chronic kidney disease and higher mortality. ${ }^{7}$

The authors' studied whether endovascular interventions for critical limb ischemia is associated with long-term loss of renal function and which individuals are at increased risk of adverse events after these interventions. The authors hypothesized that patients treated with endovascular interventions would have a significant greater annual mean renal decline, compared to patients treated with surgical interventions. 


\section{Material and Methods}

\section{Study design}

This retrospective cohort study was conducted at a peripheral hospital in the South of the Netherlands. In the period between January $1^{\text {st }} 2011$ until July $31^{\text {st }} 2013$ all new patients with critical limb ischemia treated with endovascular or surgical intervention were included. Using the outpatient clinic database patients with rutherford classification IV-VI were analysed. Rutherford class IV was defined as ischemic rest pain. Rutherford class $V$ was defined as minor tissue loss, and Rutherford class VI was defined as ulceration or gangrene. Pre-procedural eGFR and one year post-procedural eGFR difference were analysed between the two treatment groups. The eGFR was calculated from serum creatinine using the CKD-EPI. ${ }^{8}$ The lowest and shortest serum creatinine concentration in time measured before the index treatment (maximum of six months) was used to calculate baseline eGFR, and the lowest serum creatinine concentration measured between 11 and 13 months after the index treatment was used to calculate eGFR after 1 year. Additionally, acute kidney injury defined as $>50 \%$ increase in serum creatinine 3 days postoperative was analysed between the two study groups.

Exclusion criteria were end-stage renal disease (ESRD), acute ischemia, no renal function test, rutherford classification II-III and patients undergoing a second endovascular intervention, percutaneous coronary intervention or CT-angiography in the first year of follow-up. All patients underwent ankle-brachial pressure index (ABPI), duplex arterial mapping and magnetic resonance imaging (MRI) of the arterial vessels was made, followed by evaluation in an multi-disciplinary setting (vascular surgeons, intervention radiologist). In addition, all patients received statin and anti-platelet therapy. Fast renal decline was defined as more than $4 \mathrm{~mL} / \mathrm{min}$ loss of eGFR in one year. The study was carried out in accordance with the declaration of Helsinki and was approved by the institutional review board.

\section{Endovascular Procedures}

All endovascular procedures were performed by two experienced staff interventional radiologist and/or in association with one of three vascular surgeons. All procedures were 
executed in a endovascular suite equipped with a Philips Allure Xper FD20 (Philips, Eindhoven, the Netherlands) on a carbon fibre movable interventional table. Pulse beam radiation was routinely utilized. Two types of contrast agents were utilized, Xenetix 300 (Geurbet, Gorinchem, the Netherlands), in patients requiring nephroprotection or with a known allergy for contrast. All other patients received Omnipaque 240mg (GE Healthcare,

Pittsburgh, United States of America). In our hospital we utilize a national renal protective program, which includes the following; Nephrotoxic drugs (NSAIDs, diuretics, ACEinhibitors, metformin, digoxin, sotalol, lithium and colchicine) were not taken on the day of the intervention. Patients with eGFR $<45 \mathrm{~mL} / \mathrm{min}$ or with eGFR $<60 \mathrm{~mL} / \mathrm{min}$ and additional risk factors for contrast-induced nephropathy (diabetes mellitus, heart failure, age $>75$ years, hypotension, and anaemia) received pre-procedural hydration. Standard hydration regime consists of four hours of hydration with sodium-bicarbonate and highrisk cardiac patients received twelve hours of hydration with sodium-bicarbonate.

\section{Surgical intervention}

The interventions ranged from central reconstruction, such as aortic-bifurcation grafts due to aorto-iliac occlusion. Furthermore, femoral endarterectomy, femoro-femoral crossover, supra-genicular, infra-genicular and femoro-crural bypass interventions were performed.

\section{Risk factor definitions}

Heart failure was defined as a reduced ejection fraction of less than $40 \% .{ }^{9}$ Arterial hypertension was assumed when measurement of arterial blood pressure exceeded 140 $\mathrm{mm} \mathrm{Hg}$ (systolic) and/or $90 \mathrm{~mm} \mathrm{Hg}$ (diastolic) at least on two different occasions or if the patient was on anti-hypertensive medication. ${ }^{10}$ Hyperlipidaemia was defined by a total serum cholesterol level of $>5 \mathrm{mmol} / \mathrm{L}$, serum high-density lipoprotein cholesterol level of $>1 \mathrm{mmol} / \mathrm{L}$, or serum triglyceride level of $>2 \mathrm{mmol} / \mathrm{L} .{ }^{10}$ Diabetes mellitus was defined by fasting blood sugar levels $>120 \mathrm{mg} / \mathrm{dL}$, or HbA1c level $>6 \%$. In addition, presence of diabetes mellitus was assumed if the patient consumed any hypoglycaemic drugs. ${ }^{10}$ Current smoking habits were divided into either smoking or non-smoking. ${ }^{10}$ Anaemia was defined as $<8.1 \mathrm{mmol} / \mathrm{l}$ in males and $<7.5 \mathrm{mmol} / \mathrm{l}$ in females. ${ }^{11}$ Familiar vascular disease defined as a positive familiar history of vascular disease for mother-father or brother-sister. 


\section{Sample size}

Sample size was calculated assuming mean renal decline difference between the two intervention groups of $7 \mathrm{~mL} / \mathrm{min} / 1,73 \mathrm{~m}^{2}$ and a standard deviation of $11 \mathrm{~mL} / \mathrm{min} / 1.73$ $\mathrm{m}^{2}$. The type 1 error of the study was set at 0.05 and the power of the study at 0.20 . With these assumptions, sample size was calculated, requiring 77 patients for each study group. The difference in mean renal decline was based on prior research of this study group comparing patients with rutherford classification II-III, treated endovascular or non-invasive with supervised exercise therapy.

\section{Statistical analysis}

All statistical computing was carried out by T.A.S. and M.G.J.S. using SPSS version 21 (SPSS Inc., Chicago, IL, USA). Missing data were replaced utilizing multiple imputation. Continuous variables were expressed as means with standard deviations (SD) or range and categorical variables as percentages. Baseline characteristics of endovascular and surgical patients in both groups were compared with paired samples $t$ tests for continuous variables and with Fisher exact tests for categorical variables. Time-to-event analysis for survival was computed with log-rank Kaplan-Meier test. Clinical outcomes of endovascular and surgical intervention patients were compared with multivariable regression models. Adjustment for baseline characteristics and interaction terms was performed in a two-step approach. First, each baseline characteristic was added separately to multivariable regression model containing renal decline and all variables were tested for statistical significance. After univariate analyses for the confounding factors, final models were obtained by removal of interaction terms that were not statistically significantly associated with the outcome of interest. Fast renal decline was determined with multivariable logistic regression analysis using the Wald test for statistical significance. Assumptions of the regression models were checked with normal probability-probability, residual plots and by addition of quadratic terms when appropriate; these terms were retained in the model if statistically significant. Multicollinearity and influential outliers were checked using variance inflation factors and Cook's distances. Results with $P$ less than 0.05 were considered statistically significant. 


\section{Results}

A total of 290 patients, of which 209 patients were treated with endovascular procedures and 81 with surgical interventions, were included in this retrospective observational cohort study. A flow chart is presented in Figure 1 and patient characteristics are presented in Table 1.

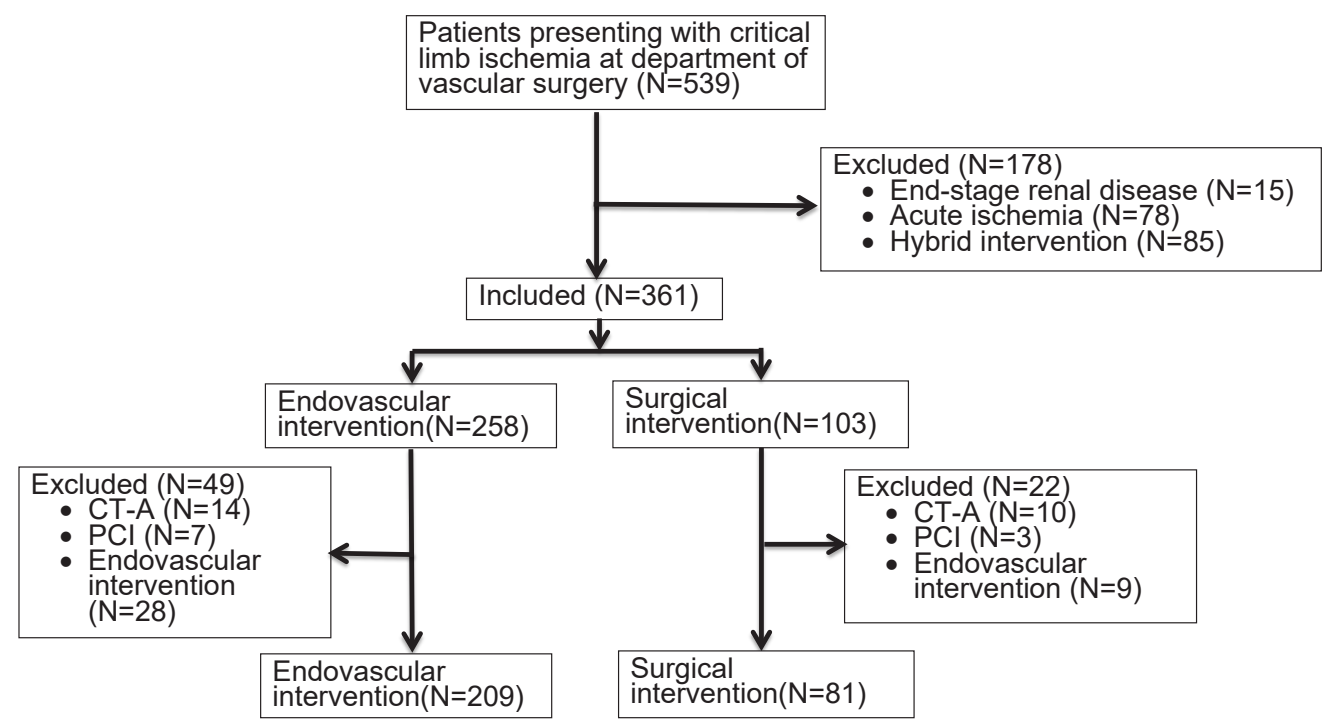

Figure 1: Flowchart of patient enrolment

Patients receiving surgical interventions were significantly more frequent male $(\mathrm{P}=0.01)$. Patients treated with endovascular procedures were more often smokers $(\mathrm{P}<0.01)$. Furthermore, a significant difference in rutherford classification exists between the two intervention groups, with more patients with rest pain being treated with surgical bypass intervention.

In patients receiving endovascular procedures for critical limb ischemia, estimated glomerular filtration rates (eGFR) decreased by $15.0 \pm 14.1 \mathrm{~mL} / \mathrm{min}(\mathrm{P}<0.001)$ in the first year after treatment (Figure 2). In the same period, renal function decreased by $7.6 \pm 10.9$ $\mathrm{mL} / \mathrm{min}(\mathrm{P}<0.001)$ in patients treated surgically. Although eGFR rates decreased in both groups, renal function loss was significantly greater in patients following endovascular interventions $(\mathrm{P}<0.001)$. Furthermore, $77 \%$ of patients receiving endovascular interventions experienced fast renal decline (defined as $>4 \mathrm{~mL} / \mathrm{min}$ loss of eGFR within 
one year) as compared to $54 \%$ of patients treated surgically $(\mathrm{P}<0.001)$. Although over $19 \%$ of the population undergoing endovascular intervention experienced acute kidney injury $(\mathrm{N}=60)$, compared with the $12 \%$ of the patients treated surgically $(\mathrm{P}=0.08)$. (Acute kidney injury was defined as a $>50 \%$ increase in serum creatinine at day 3 postoperative).

Table 1: Baseline characteristics of study subjects

\begin{tabular}{|c|c|c|c|}
\hline \multirow[t]{2}{*}{ Variables } & \multirow[t]{2}{*}{ Endovascular $(\mathrm{N}=\mathbf{2 0 9})$} & \multirow[t]{2}{*}{ Surgical $(\mathrm{N}=81)$} & \multirow[b]{2}{*}{ P-value } \\
\hline & & & \\
\hline Gender (male) & 53 & 70 & 0.01 \\
\hline Age (years) & $74[43-96]$ & $72[40-90]$ & 0.36 \\
\hline $\mathrm{BMI}\left(\mathrm{kg} / \mathrm{m}^{2}\right)$ & $27[15-50]$ & $26[15-41]$ & 0.10 \\
\hline Rutherford & & & $<0.01$ \\
\hline Classification IV & 23 & 46 & \\
\hline Classification V & 57 & 29 & \\
\hline Classification VI & 20 & 25 & \\
\hline Contrast (millilitre) & $88[24-220]$ & o & $<0.01$ \\
\hline Smoking & 62 & 40 & $<0.01$ \\
\hline Duration complaints (months) & $2.1[1-9]$ & $1.8[1-6]$ & 0.69 \\
\hline \multicolumn{4}{|l|}{ Co morbidities } \\
\hline Diabetes Mellitus & 51 & 56 & 0.51 \\
\hline $\begin{array}{l}\text { Familial vascular } \\
\text { disease }\end{array}$ & 59 & 53 & 0.33 \\
\hline Hypercholesterolemia & 57 & 44 & 0.06 \\
\hline Heart Failure & 16 & 12 & 0.40 \\
\hline Hypertension & 61 & 68 & 0.29 \\
\hline Creatinine (baseline) & $94[33-351]$ & $105[39-347]$ & 0.10 \\
\hline eGFR $\left(\mathrm{mL} / \mathrm{min} / 1.73 \mathrm{~m}^{2}\right)$ & 69 & 65 & 0.16 \\
\hline$>6 o m l$ & 71 & 62 & \\
\hline $45-59 \mathrm{ml}$ & 17 & 17 & \\
\hline $31-45 \mathrm{ml}$ & 7 & 10 & \\
\hline $16-30 \mathrm{ml}$ & 15 & 11 & \\
\hline Aorta-iliac & 30 & 10 & $<0.01$ \\
\hline Femoral-popliteal & 35 & 43 & \\
\hline Below-knee & 11 & 43 & \\
\hline Multi-level & 24 & 4 & \\
\hline
\end{tabular}

Data presented as mean (range) or as percentages. 


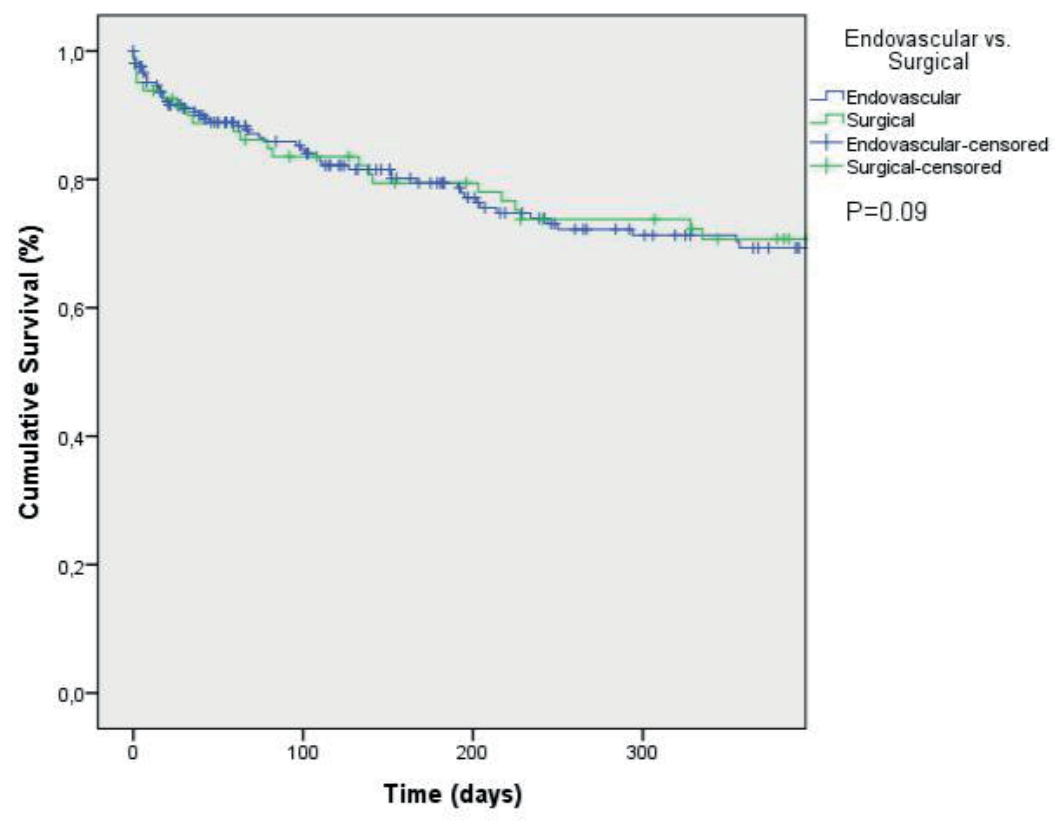

Figure 2: One year mortality of patients treated with surgical or endovascular interventions

\begin{tabular}{llllll}
\hline Numbers at risk & \multicolumn{1}{l}{} \\
\hline Time (days) & 0 & 90 & 180 & 270 & 360 \\
Endovascular intervention & 201 & 161 & 158 & 141 & 136 \\
Surgical intervention & 81 & 66 & 59 & 55 & 49 \\
\hline
\end{tabular}

Kaplan-Meier product limit estimates of survival of patients treated with endovascular and surgical interventions.

Sensitivity analysis without patients with major amputation $(\mathrm{N}=25)$ showed similar results. Respectively, in patients receiving endovascular procedures, eGFR decreased by $14.7 \pm 14.1$ $\mathrm{mL} / \mathrm{min}(\mathrm{P}<0.001)$ in the first year after treatment compared to a renal function decreased by $7.6 \pm 11.0 \mathrm{~mL} / \mathrm{min}(\mathrm{P}<0.001)$ in patients treated surgically. Furthermore, $76 \%$ of patients receiving endovascular interventions experienced fast renal decline compared to $56 \%$ of patients treated surgically $(\mathrm{P}<0.001)$. Finally, acute kidney injury occurred in $19 \%$ of the patients treated endovascular, compared to $11 \%$ in the surgically treated group $(\mathrm{P}=0.08)$. 
Multivariable linear regression was used to correct for potential confounders of the association between endovascular procedures and loss of renal function (Table 2). When clinically relevant patient characteristics were included in the analysis, endovascular intervention was associated with a $7.4 \mathrm{~mL} / \mathrm{min}$ reduction $(95 \% \mathrm{CI}: 4.4-15, \mathrm{P}<0.01)$ of eGFR and with a 6.1-fold (95\%CI: $2.4-16, \mathrm{P}<0.01)$ increased odds of fast renal decline after one year (Table 3). Additionally, when acute kidney injury was added to the multivariate regression model, endovascular intervention was associated with a $8.9 \mathrm{~mL} /$ min reduction (95\%CI: $3.8-14, \mathrm{P}<0.01)$ of eGFR and with a 9-fold (95\%CI: $3.9-$ 14.1, $\mathrm{P}<0.01)$ increased odds of fast renal decline after one year. Acute kidney injury was associated with a $10.6 \mathrm{~mL} / \mathrm{min}$ increased reduction $(95 \% \mathrm{CI}$ : $6.6-14.5, \mathrm{P}<0.01)$ in mean renal decline (Table 3). The interaction between treatment and baseline renal function was not statistically significant. Sensitivity tests without imputation of missing data showed similar results.

One year mortality results were similar for both study groups, namely $23 \%$ of the patients died in the endovascular group, compared to $27 \%$ of the patients in the surgical intervention group $(\mathrm{P}=0.09)$ [Log Rank (Mantel-Cox)] (Figure 2).

Table 2: Multivariate linear regression of loss of kidney function and multivariate logistic regression of fast renal decline

\begin{tabular}{lllllll}
\hline & \multicolumn{3}{l}{ Renal decline $(\mathrm{mL} / \mathrm{min})$} & \multicolumn{3}{l}{ Fast renal decline (yes) } \\
\hline & $\begin{array}{l}\text { Mean } \\
\text { difference }\end{array}$ & $95 \%(\mathrm{Cl})$ & $\mathbf{P}$ & OR & $95 \%(\mathrm{Cl})$ & $\mathbf{P}$ \\
\hline Endovascular intervention & 9.6 & $(4.1-15.1)$ & $<0.01$ & 6.5 & $(2.4-17.6)$ & $<0.01$ \\
Gender (male) & 1.1 & $(-2.2-4.4)$ & 0.51 & 0.9 & $(0.5-1.6)$ & 0.67 \\
Age (/years) & 0.1 & $(-0.04-0.3)$ & 0.13 & 1.0 & $(0.98-1.0)$ & 0.53 \\
Diabetes Mellitus & 0.9 & $(-4.3-2.3)$ & 0.56 & 1.1 & $(0.6-1.8)$ & 0.81 \\
Heart Failure & 5.1 & $(0.7-9.6)$ & 0.02 & 0.3 & $(0.1-0.9)$ & 0.03 \\
Hypertension & 1.6 & $(-1.8-5.1)$ & 0.39 & 1.2 & $(0.7-2.2)$ & 0.57 \\
GFR (pre-operative) & 0.1 & $(-0.08-0.1)$ & 0.85 & 1.0 & $(0.9-1.0)$ & 0.28 \\
Contrast & 0.3 & $(0.02-0.08)$ & 0.17 & 1.0 & $(0.9-1.0)$ & 0.03 \\
Rutherford & 1.1 & $(-1.2-4.0)$ & 0.34 & 0.8 & $(0.4-1.7)$ & 0.59 \\
Smoking & 0.5 & $(-3.0-4.0)$ & 0.80 & 0.9 & $(0.5-1.7)$ & 0.81 \\
\hline
\end{tabular}

Linear model of predictors of renal decline, with $95 \% \mathrm{Cl}$ interval and P-values. Coefficients of the model predicting fast renal decline, showing odds-ratio (OR), $95 \%$ confidence interval and P-values. 
Table 3: Multivariate linear regression of loss of kidney function and multivariate logistic regression of fast renal decline with acute kidney injury

\begin{tabular}{lllllll}
\hline & \multicolumn{2}{l}{ Renal decline $(\mathrm{mL} / \mathrm{min})$} & \multicolumn{3}{l}{ Fast renal decline (yes) } \\
\hline & $\begin{array}{l}\text { Mean } \\
\text { difference }\end{array}$ & $95 \%(\mathrm{Cl})$ & $\mathbf{P}$ & OR & $95 \%(\mathrm{Cl})$ & $\mathbf{P}$ \\
\hline Endovascular intervention & 8.9 & $(3.8-14.0)$ & $<0.01$ & 9.0 & $(3.9-14.1)$ & $<0.01$ \\
Gender (male) & 0.4 & $(-2.8-3.5)$ & 0.81 & 0.9 & $(0.5-1.5)$ & 0.63 \\
Age (/years) & 0.1 & $(-0.01-0.3)$ & 0.22 & 0.1 & $(-0.5-0.3)$ & 0.19 \\
Diabetes Mellitus & 0.6 & $(-2.4-3.6)$ & 0.71 & 0.6 & $(-2.4-3.7)$ & 0.68 \\
Heart Failure & 4.7 & $(0.4-8.9)$ & 0.03 & 4.7 & $(0.5-8.9)$ & 0.03 \\
Hypertension & 1.4 & $(-1.9-4.7)$ & 0.40 & 1.4 & $(-1.9-4.6)$ & 0.42 \\
GFR (pre-operative) & 0.02 & $(-0.06-0.1)$ & 0.60 & 0.02 & $(-0.1-0.1)$ & 0.59 \\
Acute kidney injury & 10.5 & $(6.5-14.5)$ & $<0.01$ & 10.6 & $(6.6-14.5)$ & $<0.01$ \\
Contrast & 0.03 & $(-0.06-0.1)$ & 0.19 & 0.03 & $(0.01-0.08)$ & $<0.01$ \\
\hline
\end{tabular}

Linear model of predictors of renal decline, with $95 \% \mathrm{Cl}$ interval and P-values. Coefficients of the model predicting fast renal decline, showing odds-ratio (OR), $95 \%$ confidence interval and P-values.

\section{Discussion}

The present study represents consecutive patients with critical limb ischemia undergoing endovascular or surgical revascularization. Patients treated endovascular experienced a significantly greater annual renal decline compared to surgically treated patients. Moreover, fast renal decline was occurred more often in the endovascular study group and a trend in acute kidney injury following endovascular revascularization was found $(\mathrm{P}=0.08)$. It is the authors opinion, that these results might have been significant when a larger population would have been investigated. While our retrospective trial was not powered to define a statistically significant difference in acute kidney injury following these interventions.

Critical limb ischemia is the most severe form of peripheral arterial disease, causing ischemic rest pain or tissue loss and is frequently correlated with chronic kidney disease. Although currently available literature suggests that there is no difference in clinical outcomes for patients with critical limb ischemia treated with endovascular or surgical revascularization, increasing amounts of patients are treated endovascular. Due to the modest outcomes and significant morbidity after infra-inguinal bypass for critical limb ischemia, many physicians prefer the less invasive endovascular intervention. It is the authors opinion 
that clinical decision making in these patients with critical limb ischemia must carefully weigh a variety of patient, anatomic and procedural variables. Factors influencing this decision include; anatomic distribution of atherosclerotic disease, lesion length, age, vein quality and comorbidities such as chronic kidney disease. Patients presenting with critical limb ischemia have higher prevalence of chronic kidney disease and comorbidities such as diabetes, hypertension, coronary artery disease, congestive heart failure, which increases with declining renal function.

A large series on endovascular outcomes for the treatment of CLI in patients with chronic kidney disease is lacking. However, a recent study describes the association between postoperative acute kidney injury after lower extremity revascularization and the incidence of chronic kidney disease and mortality. This study demonstrates that there is increased risk of long-term chronic kidney disease and mortality in patients who develop acute kidney injury after lower extremity revascularization. ${ }^{7}$

Renal function decline has been described after endovascular intervention of abdominal aortic aneurysms. The authors suggest that the pathophysiology of renal decline following endovascular interventions in CLI patients is multifactorial. Revascularization of ischemic tissue, especially an ischemic limb, results in a number of systemic problems characterized by metabolic acidosis, hyperkalemia, myoglobinemia, myoglobinuria, and renal failure. ${ }^{12}$

When blood flow is restored to an ischemic limb, acidic blood is returned to the circulation resulting in metabolic acidosis. In addition to contrast use, drug toxicity (ie, antibiotics), and possible embolization are likely causes of acute kidney injury in these individuals. All this does not explain the long-term renal decline in this patient group. Animal studies provide some insight into possible mechanisms for delayed chronic kidney disease development after acute kidney injury recovery. Although the regenerative ability of tubular epithelial cells is well established, the capacity for vascular endothelial recovery appears to be more limited, and significant vascular dropout has been observed after ischemic acute kidney injury in animal models. ${ }^{13}$ These changes appear to be mediated by loss of vascular endothelial growth factor protection. ${ }^{14}$ Despite the improvement in kidney function after a documented acute kidney injury, there seems to be a persistent vascular damage that may contribute to the risk of long-term renal decline and the development of end-stage renal disease. These results are in concurrence with our study findings indicating the substantial need for renal function monitoring following contrast administration, especially in this high-risk population with critical limb ischemia. 
In contrast to the traditional paradigm, loss of kidney function is not necessarily constant over time. Many patients with chronic kidney disease have non-linear trajectories of renal decline with prolonged periods of stable renal function interrupted by episodes of fast renal decline. This study reports that between 50-75\% experienced considerable and clinically significant renal function decline one year post-procedural. These changes are clinically significant even when an expected $8 \%$ daily variation in serum creatinine is taken into consideration. ${ }^{15}$

Renal dysfunction should therefore be considered a common sequel of critical limb revascularization and it deserves focused attention as a therapeutic target in efforts to improve long-term survival. Several reports have shown that larger creatinine elevations after hospitalization predict highest risk of death and even minor changes in renal function are associated with adverse outcomes. The choice of a 'best definition' for worsening renal function has implications for the number of patients identified with this risk factor and the magnitude of risk for mortality. ${ }^{16}$ A number of scores have been proposed as tools for assessing kidney injury in the acute phase, such as RIFLE and AKIN; however, there is no consensus on an appropriate longer-term method as yet. Additionally, several reports describe fast renal decline (eGFR loss $>4 \mathrm{~mL} / \mathrm{min}$ annually) as increased risk for adverse events and death.

A need exists to quantitatively summarize incidence and severity of renal dysfunction after endovascular interventions in patients with critical limb ischemia to allow better-informed attempts to preserve renal function and improve life expectancy. Strategies to preserve renal function therefore represent a logical therapeutic target for improving life expectancy. The etiology of postoperative renal dysfunction is almost certainly multifactorial, including baseline renal function, diabetes mellitus, congestive heart failure, hyponatremia, previous diuretic usage, hypoalbuminemia, age, and contrast dose. ${ }^{17}$ Based on the severely reduced long-term survival rates of patients with critical limb ischemia and chronic kidney disease, future efforts should focus on early detection of peripheral arterial disease in patients with renal insufficiency and institution of aggressive medical therapy to prevent progression in the global burden of atherosclerosis in this patient population.

Some limitations of this study need to be acknowledged, first our cohort predominantly consisted of Caucasian patients. It is known that renal function differs between races, limiting the generalizability of the outcome to a wider variability in the general population. Secondly, as in all observational studies, confounders may have biased our results. 
However, corrections for the most important confounders that have been reported in previous publications by multivariate analysis did not change the findings. Therefore, we do not expect that unmeasured confounders would significantly impact on our findings. Finally, our measurement of acute kidney injury at day 3 post-procedural does not meet the AKIN-criteria for acute kidney injury. ${ }^{2}$ However, our study group has chosen day 3 while this report compared surgically and endovascularly treated patients. Moreover, contrast-induced nephropathy characteristically manifests 3 days after administration of the contrast medium, with a peak in renal function decline 3 to 5 days after contrast exposure. $^{18}$

\section{Conclusion}

Endovascular procedures for critical limb ischemia are associated with clinically relevant permanent long-term loss of kidney function. This loss of renal function is greater than in comparable patients who were treated with open surgery. 


\section{References}

1. Bucaloiu ID, Kirchner HL, Norfolk ER, Hartle JE 2nd, Perkins RM. Increased risk of death and de novo chronic kidney disease following reversible acute kidney injury Kidney International 2012;81: 477-485

2. KDIGO Clinical Practice Guideline for Acute Kidney Injury official journal of the international society of nephrology March 2012 Volume 2 Issue 1 http://www.kidney-international.org

3. Loef BG, Epema AH, Smilde TD, Henning RH, Ebels T, Navis G, et al. Immediate postoperative renal function deterioration in cardiac surgical patients predicts in-hospital mortality and long-term survival. J Am Soc Nephrol 2005;16:195-200.

4. Uchino S, Kellum JA, Bellomo R, Doig GS, Morimatsu H, Morgera S, et al. Acute renal failure in critically ill patients: a multinational, multicenter study. JAMA 2005;294:813-8.

5. Wang HE, Muntner P, Chertow GM, Warnock DG. Acute kidney injury and mortality in hospitalized patients. Am J Nephrol 2012;35:349-55.

6. Whaley-Connell AT, Sowers JR, Stevens LA, McFarlane SI, Shlipak MG, Norris KC, et al. CKD in the United States: Kidney Early Evaluation Program (KEEP) and National Health and Nutrition Examination Survey (NHANES) 1999-2004. Am J Kidney Dis 2008;51(4 Suppl2):S13-20.

7. Arora P, Davari-Farid S, Pourafkari L, Gupta A, Dosluoglu HH, Nader ND. The effect of acute kidney injury after revascularization on the development of chronic kidney disease and mortality in patients with chronic limb ischemia J Vasc Surg 2015;61:720-7

8. Levey AS, Stevens LA, Schmid CH, Zhang YL, Castro AF $3^{\text {rd }}$, Feldmnad HI, et al. A New Equation to Estimate Glomerular Filtration Rate. Ann Intern Med. 2009; 150:604-612.

9. "Ejection Fraction Heart Failure Measurement". American Heart Association. Feb 11, 2014.

10. Diehm N, Shang A, Silvestro A, Do DD, Dick F, Schmidli J, et al. Association of cardiovascular risk factors with pattern of lower limb atherosclerosis in 2659 patients undergoing angioplasty. Eur J Vasc Endovasc Surg 2006;31:59-63

11. WHO. Nutritional anaemias: Report of a WHO group of experts. Geneva: World Health Organisation, 1972. Technical report series no. 503.

12. Morsey H, Aslam M, Standfield N. Patients with critical ischemia of the lower limb are at risk of developing kidney dysfunction Am J Surg. 2003 Apr;185(4):360-3.

13. Horbelt M, Lee SY, Mang HE, Knipe NL, Sado Y, Kribben A, et al. Acute and chronic microvascular alterations in a mouse model of ischemic acute kidney injury. Am J Physiol Renal Physiol 2007;293:F688-95.

14. Basile DP, Friedrich JL, Spahic J, Knipe N, Mang H, Leonard EC, et al. Impaired endothelial proliferation and mesenchymal transition contribute to vascular rarefaction following acute kidney injury. Am J Physiol Ren Physiol 2011;300:F721-33.

15. Traynor J, Mactier R, Geddes CC, Fox JG. How to measure renal function in clinical practice. BMJ 2006;333:733-737 
16. Smith GL, Vaccarino V, Kosiborod M, Lichtman JH, Cheng S, Watnick SG, et al. Worsening renal function: what is a clinically meaningful change in creatinine during hospitalization with heart failure? J Card Fail 2003;9: 13-25

17. Walsh SR, Tang TY, Boyle JR. Renal consequences of endovascular abdominal aortic aneurysm repair. J Endovasc Ther 2008;15:73-82

18. Murphy SW, Barrett BJ, Parfrey PS, Contrast nephropathy. J Am Soc Nephrol 2000;11(1):17782 


\section{снартів 4 Radiation exposure during}

percutaneous transluminal angioplasty for symptomatic peripheral arterial disease

Tim A Sigterman ${ }^{1}$, Lars JJ Bolt ${ }^{1}$, Attila G Krasznai ${ }^{1}$, Maarten G Snoeijs ${ }^{1}$, Roel Heijboer ${ }^{1}$, Geert-Willem H. Schurink², LH. Bouwman ${ }^{1}$

1. Zuyderland Medical Centre, Heerlen, the Netherlands 2. Maastricht University Medical Centre, Maastricht, the Netherlands

Published in:

Ann Vasc Surg. 2016 May;33:167-72 


\section{Abstract}

Background. Endovascular interventions often are the preferred treatment in peripheral arterial disease for both intermittent claudication and chronic critical limb ischemia. Ever more advanced and anatomically complex procedures are being performed, which may increase the radiation exposure of patients and medical staff. We sought to determine the radiation exposure sustained in contemporary interventional radiology practice.

Methods. A single-centre observational cohort study of patients undergoing endovascular interventions for symptomatic peripheral arterial disease between January 1st 2011 and July 31 st 2013 was performed. Radiation dose area product, peak skin dose, fluoroscopy time and volume of contrast media were either measured or calculated and stratified according to patient demographics and anatomical location.

Results. In 951 patients who were treated with endovascular interventions, mean dose area product was $108 \pm 88 \mathrm{~Gy}^{*} \mathrm{~cm}^{2}$ and mean fluoroscopy time was $13 \pm 12$ minutes. Dose area product was significantly higher for aorto-iliac procedures $\left(142 \pm 103 \mathrm{~Gy}^{*} \mathrm{~cm}^{2}\right)$ compared to femoral $\left(58 \pm 73 \mathrm{~Gy}^{*} \mathrm{~cm}^{2}\right)$, below-knee $\left(45 \pm 51 \mathrm{~Gy}^{*} \mathrm{~cm}^{2}\right)$ and multi-level $\left(83 \pm 90 \mathrm{~Gy}^{*} \mathrm{~cm}^{2}\right)$ procedures $(\mathrm{P}<0.001$ for all comparisons). Peak skin dose was significantly higher for aorto-iliac procedures $(0.99 \pm 0.54 \mathrm{~Gy})$ compared to femoral $(0.55 \pm 0.55 \mathrm{~Gy})$, below-knee $(0.48 \pm 0.27 \mathrm{~Gy})$ and multi-level $(0.68 \pm 0.47 \mathrm{~Gy})$ procedures ( $\mathrm{P}<0.001$ for all comparisons). In contrast, mean fluoroscopy time in aorto-iliac interventions was significantly shorter $(10 \pm 15 \mathrm{~min})$ compared to femoral $(15 \pm 12 \mathrm{~min})$, below-knee $(19 \pm 10 \mathrm{~min})$ and multi-level $(23 \pm 14 \mathrm{~min})$ procedures $(\mathrm{P}<0.001$ for all comparisons).

Conclusions. Endovascular interventions in patients with symptomatic peripheral arterial disease involve a substantial radiation dose. Even though fluoroscopy time is shorter, aorto-iliac interventions result in greater radiation exposure than femoropopliteal or infrapopliteal interventions. 


\section{Introduction}

Vascular surgeons and interventional radiologists perform an increasing number of endovascular interventions for peripheral arterial disease, leading to cumulative radiation exposure for patients and medical staff. Radiation exposure can lead to deterministic effects that primarily affect the skin. Once a radiation threshold of $2 \mathrm{~Gy}$ is exceeded, the damage to tissue will become more severe with increasing dose. ${ }^{1}$ Manifestations range from erythema to severe lesions like acute ulceration of the skin and often occur within 24 hours of the intervention. Stochastic effects do not depend on a certain threshold to be exceeded, but might occur after any given dose. The most important stochastic manifestations are radiation-induced malignancies. The possibility of a radiation-induced fatal malignancy due to an interventional procedure is estimated to be less than $0.5 \%{ }^{1}$ Moreover, there is convincing epidemiological evidence that doses of ionizing radiation above about a few tens of $\mathrm{mGy}$ cause a small but significant increase in cancer risk. ${ }^{2}$

Few studies have documented radiation exposure with regard to stenosis location in patients undergoing endovascular interventions. ${ }^{3,4}$ One small single center study reported the radiation dose in percutaneous interventions in patients with intermittent claudication (IC) and/or critical limb ischemia (CLI). ${ }^{5}$ In this report, patients with aortoiliac stenotic lesions received the highest radiation dose, compared to femoropopliteal and below-knee anatomic levels. However, in this study patient body mass index was not recorded routinely, even though literature has stated that higher body mass index (BMI) is a predictor for increased radiation. ${ }^{6}$ Procedural characteristics associated with higher radiation dose include lesion location, procedure type and case complexity. ${ }^{7,8}$

The aim of this study was to analyse radiation doses during endovascular intervention at different anatomic levels in patients with symptomatic peripheral arterial disease. The authors hypothesized that aorto-iliac interventions would have a substantially higher radiation exposure compared to other, more distal anatomic levels. 


\section{Methods}

Meeting all criteria of the local medical ethical committee a retrospective cohort study was conducted at a peripheral hospital in the South of the Netherlands. In the period between January $1^{\text {st }} 2011$ until July $31^{\text {st }} 2013$ all patients with intermittent claudication or critical limb ischemia treated with endovascular interventions were included. Exclusion criteria were thrombolysis, endovascular interventions of visceral arteries and arterio-venous fistulas.

Procedures were divided into four separate groups, according to anatomic location:

- Aorto-iliac: (Aorta, common iliac, internal iliac, external iliac and common femoral artery)

- Femoral: (Superficial femoral and popliteal artery)

- Below-knee: (Tibioperoneal trunk, anterior tibial, posterior tibial, peroneal artery)

- Multi-level: (procedures involving any combination of the above including endovascular intervention of prior surgical bypass, in case of prior bypass the anatomic location was depicted were the intervention was performed. In case of supragenual femoropopliteal bypass the anatomic location was femoral)

\section{Endovascular intervention}

All endovascular interventions were performed by two interventional radiologists or vascular surgeons in an endovascular theatre provided with a Philips Allura Xper FD20 fixed X-ray system (Philips, Eindhoven, the Netherlands) and an adjustable carbon fiber interventional table. Imaging through radiation with pulsed beam was routinely used. The field of view of the image intensifier was adjusted according to the procedure, ranging from 17 to 48 centimeters. Collimation and filtering were applied as much as possible.

After completion of the intervention the radiation dose, fluoroscopy time and contrast volume were routinely recorded in the patient $\log$ of the endovascular theatre. Radiation dose was defined as the cumulative dose area product (DAP; measured in $\mathrm{Gy}^{*} \mathrm{~cm}^{2}$ ). This is the complete dose a patient is exposed to during the endovascular procedure, calculated by the X-ray system. Fluoroscopy time is the total time in minutes, however during substraction no fluoroscopy time is measured. The frames per second (FPS) are for iliac 
three second with 2 frames per second, for femoral and below-knee 8 seconds with 1 frame per second. Finally, the contrast dose was predominantly given by the "Medrad injector", which calculates the amount of contrast given during the procedure. This volume was added by the amount of contrast given by the operator manually. Mean peak skin dose (PSD) (Gy) was derived from the dose area product $\left(\mathrm{Gy}^{*} \mathrm{~cm}^{2}\right)$ using a conversion formula ${ }^{1}$ : PDS $(\mathrm{mGy})=249+5.2 *$ DAP $\left(\mathrm{Gy}^{*} \mathrm{~cm}^{2}\right)$. As no significant differences were identified between the dose area product values and fluoroscopy time of the various physicians performing the procedures, a pooled analysis was undertaken.

\section{Statistical analyses}

Statistical analyses were executed using SPSS version 19.0 (SPSS, Chicago, IL, USA). Continuous variables were presented as means and range as indicated by the distribution of the data according to Kolmogorov-Smirnov tests. Categorical variables were presented as percentages. Differences between groups for categorical variables were evaluated using Fisher exact tests. To account for potential confounders, radiation dose, fluoroscopy time, peak skin dose and contrast volume were compared with multivariable linear regression analysis including baseline characteristics. Clinically relevant interaction terms were added to the regression models and retained in case of statistical significance. Assumptions of the regression models were checked with normal probability-probability and residual plots and by addition of quadratic terms when appropriate; these terms were retained in the model if statistically significant. Furthermore, multicollinearity and influential outliers were checked using variance inflation factors and Cook's distance. The level of significance was determined as $P<0.05$.

\section{Results}

In total 951 patients were included in the present study with a mean age of 68 years. The average BMI of the study population was $27 \mathrm{~kg} / \mathrm{m}^{2}$. Over $50 \%$ of the patients in the cohort were active smokers. Baseline characteristics of the 951 patients are shown in Table 1. 
Table 1: Baseline characteristics of the study population.

\begin{tabular}{ll}
\hline & $(\mathrm{N}=951)$ \\
\hline Gender (male) & 60 \\
Age $($ years $)$ & $68[37-96]$ \\
BMI $\left(\mathrm{kg} / \mathrm{m}^{2}\right)$ & $27[15-51]$ \\
Rutherford & \\
Classification III & 57 \\
Classification IV & 11 \\
Classification V & 25 \\
Classification VI & 9 \\
Procedure & \\
Aorto-iliac & 56 \\
Femoral & 26 \\
Below-knee & 5 \\
Multi-level & 12 \\
Co morbidities & \\
Diabetes Mellitus & 31 \\
Familiar vascular disease & 55 \\
Hypercholesterolemia & 59 \\
Congestive heart failure & 6 \\
Hypertension & 67 \\
\hline
\end{tabular}

Continuous variables are shown as mean with range and categorical variables are shown as percentages.

\section{Radiation dose}

Mean dose area product (DAP) for all interventions was $108 \mathrm{~Gy} \cdot \mathrm{cm}^{2}$ (range 0.3-631). Dose area product was significantly higher for aorto-iliac procedures $\left(142 \pm 103 \mathrm{~Gy}^{*} \mathrm{~cm}^{2}\right)$ compared to femoral $\left(58 \pm 73 \mathrm{~Gy}^{*} \mathrm{~cm}^{2}\right)$, below-knee $\left(45 \pm 51 \mathrm{~Gy}^{*} \mathrm{~cm}^{2}\right)$ and multi-level $\left(83 \pm 90 \mathrm{~Gy}^{*} \mathrm{~cm}^{2}\right)$ procedures $(\mathrm{P}<0.001$ for all comparisons) (Figure 1). 


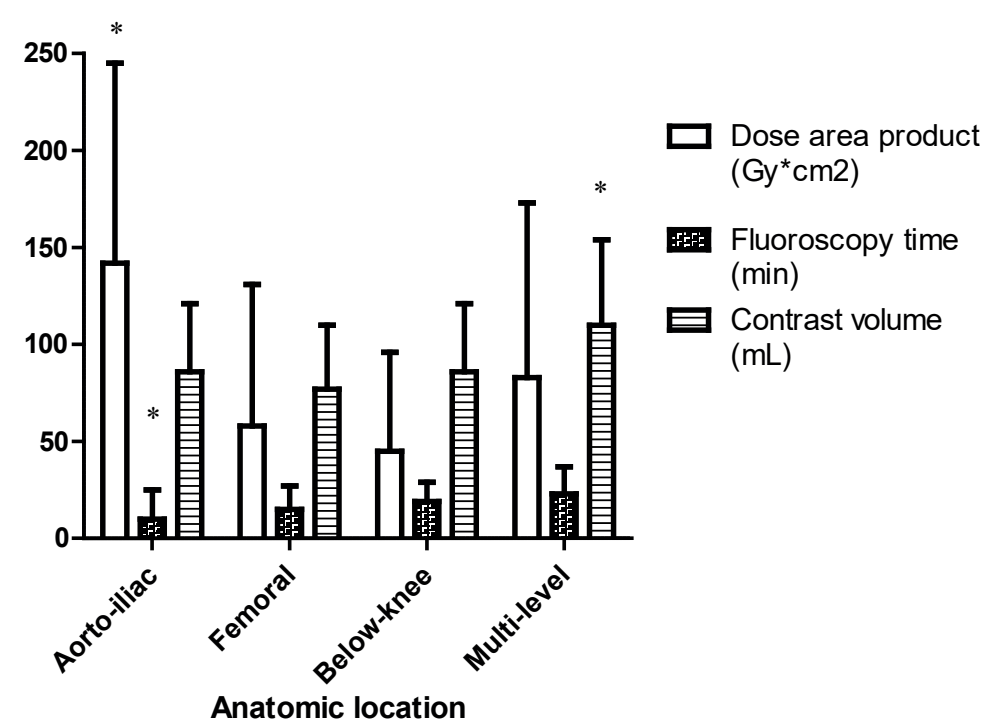

Figure 1: Radiation dose, fluoroscopy time and contrast volume in endovascular interventions for peripheral arterial disease according to anatomic location.

Data are presented as mean with standard deviation and error bars. ${ }^{*}$ depicts significant difference of aorto-iliac anatomic region compared to all other levels $(\mathrm{P}<0.001)$

Six patients received over $400 \mathrm{~Gy}^{*} \mathrm{~cm}^{2}$ and one of these patients received more than 600 $\mathrm{Gy}^{*} \mathrm{~cm}^{2}$. All these interventions were bilateral iliac interventions, three of which concerned recanalization. The other three procedures were complicated kissing stent procedures with multiple stents. All these patients had a BMI $>30 \mathrm{~kg} / \mathrm{m}^{2}$. Peak skin dose (PSD) for all interventions was 0.81 Gy (range $0.25-3.53$ ). PSD was significantly higher for aorto-iliac procedures $(0.99 \pm 0.54 \mathrm{~Gy})$ compared to femoral $(0.55 \pm 0.55 \mathrm{~Gy})$, below-knee $(0.48 \pm 0.27 \mathrm{~Gy})$ and multi-level $(0.68 \pm 0.47 \mathrm{~Gy})$ procedures $(\mathrm{P}<0.001$ for all comparisons) (Table 2). 
Table 2: Dose area product $\left(\mathrm{Gy}^{\star} \mathrm{cm}^{2}\right)$, Peak skin dose (Gy), Fluoroscopy time (min), Contrast volume (ml).

\begin{tabular}{llllll}
\hline & Aorto-iliac & Femoral & Below-knee & Multi-level & p-values \\
\hline DAP $\left(\mathrm{Gy}^{*} \mathrm{~cm}^{2}\right)$ & $142 \pm 103$ & $58 \pm 73$ & $45 \pm 51$ & $83 \pm 90$ & $<0.001^{*}$ \\
PSD $(\mathrm{Gy})$ & $0.99 \pm 0.54$ & $0.55 \pm 0.38$ & $0.48 \pm 0.27$ & $0.68 \pm 0.47$ & $<0.001^{*}$ \\
Fluoroscopy time (min) & $10 \pm 15$ & $15 \pm 12$ & $19 \pm 10$ & $23 \pm 14$ & $<0.001^{*}$ \\
Contrast $(\mathrm{ml})$ & $86 \pm 35$ & $77 \pm 33$ & $86 \pm 35$ & $110 \pm 44$ & $<0.001^{\star *}$ \\
\hline
\end{tabular}

All variables are shown as mean with standard deviation.

* For all comparisons

** Multi-level interventions compared with all other procedures.

Multivariate linear regression was used to correct for potential confounders of the association between anatomic location and radiation dose, fluoroscopy time, and contrast volume. In this analysis, body mass index was associated with a 4 fold (95\%CI: 2.6$5.3)$ increased odds for radiation dose $(\mathrm{P}<0.001)$. Moreover, critical limb ischemia was associated with a 17 fold (95\%CI:-1.8 - 32.1) increased odds as compared to intermittent claudication $(\mathrm{P}=0.03)$. And

aorta-iliac procedures were associated with a 69 fold (95\%CI: 52.6 - 85.1) increased odds as compared to femoral intervention, and with a 94 fold (95\%CI: 64.1 - 124.5) increased odds compared to below-knee and with a 74 fold (95\%CI: 51.5 - 96.6) increased odds compared to multi-level ( $\mathrm{P}<0.01$, for all comparisons). Finally, contrast volume was also associated with a 1.1 fold (95\%CI: 0.9 - 1.3) increased odds for higher radiation dose $(\mathrm{P}<0.001)$ (Table 3). 
Table 3: Multivariate linear regression for radiation dose (Gy)

\begin{tabular}{llll}
\hline Variables & Radiation dose (Gy) & \\
\cline { 2 - 3 } & $\begin{array}{l}\text { Mean } \\
\text { difference }\end{array}$ & $95 \%(\mathrm{Cl})$ & $\mathrm{P}$ \\
\hline Age (/years) & -0.40 & $(-1.00-0.30)$ & 0.24 \\
Gender (male) & -7.00 & $(-20.6-6.7)$ & 0.32 \\
BMI (/kg/m $\left.{ }^{2}\right)$ & 4.00 & $(2.60-5.40)$ & $<0.01$ \\
Rutherford & & & - \\
Intermittent claudication(ref) & - & - & 0.03 \\
Critical limb ischemia & 17.0 & $(-1.80-32.1)$ & $<0.01$ \\
Aorto-iliac vs Femoral & 68.9 & $(52.6-85.1)$ & $<0.01$ \\
Aorto-iliac vs Below-Knee & 94.3 & $(64.1-124.5)$ & $<0.01$ \\
Aorto-iliac vs Multi-level & 74.0 & $(51.5-96.6)$ & $<0.01$ \\
Contrast (mL) & 1.11 & $(0.90-1.30)$ & 0.75 \\
Fluoroscopy time (min) & 0.07 & $(-0.40-0.54)$ & 0.12 \\
GFR (pre-operative mL/min) & -0.30 & $(-0.67-0.08)$ & 0.54 \\
Diabetes mellitus (yes) & 4.70 & $(-10.5-19.8)$ & \\
\hline
\end{tabular}

Linear model of predictors of radiation dose, with $95 \% \mathrm{Cl}$ interval and P-values.

\section{Fluoroscopy time}

Mean fluoroscopy time was 13 minutes, ranging from 1 to 125 minutes. Nine patients were subject to more than 60 minutes of radiation. Mean fluoroscopy time in aortoiliac interventions was significantly shorter, $(10 \pm 15 \mathrm{~min})$ compared to femoral $(15 \pm 12$ $\mathrm{min})$, below-knee $(19 \pm 10 \mathrm{~min})$ and multi-level $(23 \pm 14 \mathrm{~min})$ procedures $(\mathrm{P}<0.001$ for all comparisons).

\section{Contrast}

The highest volume of radiocontrast was administered during multi-level interventions $(110 \pm 44 \mathrm{~mL} ; \mathrm{P}<0.001$ for comparisons with all other procedures). Aorto-iliac interventions required significantly more radiocontrast than femoropopliteal procedures ( $86 \pm 35$ vs $77 \pm 33 \mathrm{~mL}, \mathrm{P}<0.01$ ), and a similar amount of radiocontrast as infrapopliteal procedures $(86 \pm 35 \mathrm{~mL}, \mathrm{P}=0.99)$. Additional analysis for the amount of contrast utilized 
during endovascular interventions was performed. This analysis revealed a significant association between contrast volume and age, $0.32(0.07-0.6)(\mathrm{P}=0.04)$, and for critical limb ischemia, respectively a 5.7 fold $(0.4-11.1)$ increased odds compared to intermittent claudication for contrast volume $(\mathrm{P}<0.01)$. Finally, radiation dose was associated with an increased odds for contrast volume, respectively $0.15(0.12-0.17)(\mathrm{P}<0.01)$.

\section{Discussion}

The aim of this study was to analyse procedure-specific patient radiation doses during endovascular interventions for symptomatic peripheral arterial disease. This report indicates that dose area product (DAP) and peak skin dose (PSD) are both significantly higher for aorto-iliac procedures compared to femoropopliteal, infra-popliteal and multilevel interventions. Patients who received these high doses suffered from obesity (BMI $>30 \mathrm{~kg} / \mathrm{m}^{2}$ ) and they underwent relatively complex procedures. However, fluoroscopy time was significantly shorter in aorto-iliac interventions (10 min), compared to all other anatomic levels $(\mathrm{p}<0.001)$. The highest volume of radiocontrast was administered during multilevel interventions $(\mathrm{P}<0.001)$ and aorto-iliac interventions required significantly more radiocontrast than femoropopliteal procedures and a similar amount of radiocontrast as infrapopliteal procedures.

Aorto-iliac interventions are associated with increased radiation dose because more tissue has to be penetrated in the aorto-iliac region. Our findings are in line with a smaller study that reported significantly higher DAP in procedures performed in the aorto-iliac region as compared to femoropopliteal and infrapopliteal procedures. ${ }^{5}$ However, in the previous article body mass index was not routinely measured, even though literature states that patients with morbid obesity (BMI>30) treated with endovascular aortic aneurysm repair receive significantly more radiation compared to patients with a normal BMI $(<25 \mathrm{~kg} /$ $\left.\mathrm{m}^{2}\right){ }^{6}$ These findings illustrate that this report is in concurrence with current literature and the first to report body mass index as a significant predictor for higher radiation dose in patient treated with endovascular interventions for peripheral arterial disease. Furthermore, this report is the first to describe the amount of contrast utilized in different anatomic regions in patients with symptomatic peripheral arterial disease.

During endovascular interventions, patient radiation doses are routinely measured. Radiation dose can be expressed in DAP, the entire amount of energy delivered to the 
patient, measured in $\mathrm{Gy}^{*} \mathrm{~cm}^{2}$. Although DAP is an indirect estimation of radiation exposure, it is relatively accurate and a good quantity for estimating the stochastic risk for the patient. ${ }^{10,11} \mathrm{PSD}$ is another form of dosimetry that calculates the maximum radiation dose to any point on the patients' skin. PSD has several limitations: it is an indirect estimation using the dose area product and any inaccuracy of this measurement will translate directly to the PSD. Moreover, in peripheral interventions, the focus of radiation is not fixed on one area of interest and true PSD is therefore likely to be lower than the estimated values. This is mainly in aorto-iliac interventions were the C-arm rotates several times from position, respectively left and right anterior oblique views. Although fluoroscopy time has long served as a convenient technique

to estimate the amount of radiation dose, technical variations such as beam intensity, beam energy, beam orientation, field size and distance from the skin can affect the delivered dose. Therefore, radiation doses reported in this article are expressed as DAP. Novel techniques such as displacements per atom kinetic energy released in material and skin dose mapping offer better estimates. However, these novel techniques are not readily available and have their own limitations. ${ }^{12,13}$ Finally, the effect of backscatter (spreading of the radiation beyond the point that needs to be radiated) is ignored, and the actual skin dose should be estimated $10 \%-40 \%$ higher, depending on the beam area and energy. ${ }^{10}$

Negative side effects of endovascular procedures due to radiation can manifest in a direct or indirect manner. Direct deterministic manifestations become apparent once a certain radiation threshold is exceeded. Deterministic effects of radiation can occur when the threshold mean entrance skin dose of $2 \mathrm{~Gy}$ is exceeded. ${ }^{1}$ The calculated (mean) PSD values were the highest in aorto-iliac procedures $(0.99 \mathrm{~Gy}, 95 \% \mathrm{CI} ; 0.94-1.03)$. The average dose did not exceed 2 Gy and were well within acceptable limits, while $4 \%(\mathrm{~N}=35$, range $2.01-3.53$ ) received radiation doses of more than $2 \mathrm{~Gy}$. This subgroup of patients might have been affected by deterministic effects. Diabetes mellitus, smoking, and fair skin promote and accelerate skin reactions after radiation exposure. Moreover, collagen vascular disease, scleroderma and mixed connective tissue disease have been associated with increased susceptibility to skin injury at lower doses. ${ }^{14,15}$ Indirect stochastic effects are not immediately apparent and physicians should therefore be aware of the long latent period before these effects occur. Due to the increasing volume and complexity of endovascular procedures and the increasing number of obese patients, physicians should be mindful of the negative effects of a single dose of radiation. ${ }^{16}$ Moreover, an ever-growing number of patients will undergo diagnostic studies and re-interventions, leading to cumulative 
radiation exposure, which further increases the risks of stochastic side effects.

Not only patients but also medical staff are repeatedly exposed to the hazardous effects of radiation. Depending on the position of the physician, radiation exposure varies by a factor 40. ${ }^{17}$ In aorto-iliac procedures, the operator has to stand close to the lesion; subsequently the surgeon is more intensely exposed to scatter. Furthermore, hand doses are higher in aorto-iliac procedures than in femoral and below-knee interventions. ${ }^{18}$ Our data indicate that although radiation time is shortest in aorto-iliac procedures, radiation dose in aortoiliac procedures is higher than in femoropopliteal and infrapopliteal interventions. The best prevention for radiation injury to both patient and medical staff is to minimize total fluoroscopy time, keep the image intensifier close to the patient and limited to the region of interest, and use appropriate radiation shielding and monitoring. ${ }^{19}$ Furthermore, reducing magnification modes and steep gantry angulation can limit radiation dose. ${ }^{19}$

\section{Conclusion}

Endovascular interventions for peripheral arterial disease are associated with significant radiation exposure. Even though procedure times are shorter, radiation dose is higher in aorto-iliac interventions as compared to femoropopliteal and infrapopliteal procedures. The increasingly prominent role of endovascular interventions for peripheral arterial disease may increase the long-term risk of cancer in patients and medical staff. Furthermore, obese patients undergoing complex aorto-iliac interventions may be at risk of local skin effects of high radiation doses. 


\section{References}

1. Stecker MS, Balter S, Towbin RB, Miller DL, Vañó E, et al. Guidelines for patient radiation dose management. J Vasc Interv Radiol JVIR. 2009;20:S263-73.

2. Buzatu S, Cellular low-dose effects of ionizing radiation. Riv Biol. 2008;101(2):279-98)

3. D'Ercole L, Mantovani L, Thyrion FZ, Bocchiola M, Azzaretti A, et al. A study on maximum skin dose in cerebral embolization procedures. AJNR Am J Neuroradiol. 2007 Mar;28(3):503-7

4. Einstein AJ, Moser KW, Thompson RC, Cerqueira MD, Henzlova MJ. Radiation dose to patients from cardiac diagnostic imaging. Circulation. 2007;116(11):1290-305.

5. Segal E, Weinberg I, Leichter I, Klimov A, Giri J, et al. Patient radiation exposure during percutaneous endovascular revascularization of the lower extremity. I Vasc Surg. 2013;58(6):1556-62

6. Ketteler ER, Brown KR. Radiation exposure in endovascular procedures. J Vasc Surg. 2011 Jan;53:35S - 38S.

7. Bor D, Sancak T, Toklu T, Olgar T, Ener S. Effects of radiologists' skill and experience on patient doses in interventional examinations. Radiat Prot Dosimetry. 2008;129(1-3):32-5

8. Miller DL, Balter S, Cole PE, Lu HT, Schueler BA, et al. Radiation doses in interventional radiology procedures: the RAD-IR study: part I: overall measures of dose. J Vasc Interv Radiol JVIR. 2003;14(6):711-27

9. Koenig TR, Wolff D, Mettler FA, Wagner LK. Skin injuries from fluoroscopically guided procedures: part 1, characteristics of radiation injury. AJR Am J Roentgenol. 2001 Jul;177(1):311

10. Molyvda-Athanasopoulou E, Karlatira M, Gotzamani-Psarrakou A, Koulouris C, Siountas A. Radiation exposure to patients and radiologists during interventional procedures. Radiat Prot Dosimetry. 2011;147(1-2):86-9

11. Zorzetto M, Bernardi G, Morocutti G, Fontanelli A. Radiation exposure to patients and operators during diagnostic catheterization and coronary angioplasty. Cathet. Cardiovasc. Diagn 1997;40:348-

12. Stern S. Nationwide Evaluation of X-ray Trends (NEXT), Tabulation and Graphical Summary of 2000 Survey of Computed Tomography [Internet]. Conference of Radiation Control Program Directors, Inc.; 2007

13. Sarac TP, Altinel O, Bannazadeh M, Kashyap V, Lyden S, et al. Midterm outcome predictors for lower extremity atherectomy procedures. J Vasc Surg. 2008;48(4):885-890

14. Vano E, Goicolea J, Galvan C, Gonzalez L, Meiggs L, et al. Skin radiation injuries in patients following repeated coronary angioplasty procedures. Br J Radiol. 2001;74(887):1023-31.

15. Wagner LK, McNeese MD, Marx MV, Siegel EL. Severe skin reactions from interventional fluoroscopy: case report and review of the literature. Radiology. 1999;213(3):773-6

16. Reed AB. Introduction. Radiation safety in vascular surgery. J Vasc Surg. 2011;53:1S - 2S.

17. Theocharopoulos N, Damilakis J, Perisinakis K, Manios E, Vardas P, et al. Occupational 
exposure in the electrophysiology laboratory: quantifying and minimizing radiation burden. Br J Radiol. 2006;79(944):644-51.

18. Kim KP, Miller DL. Minimising radiation exposure to physicians performing fluoroscopically guided cardiac catheterisation procedures: a review. Radiat Prot Dosimetry. 2009;133(4):22733.

19. Mitchell EL, Furey P. Prevention of radiation injury from medical imaging. J Vasc Surg. 2011;53(1 Suppl):22S - 27S. 


\section{сантвв: Duplex-guided}

\section{versus conventional}

percutaneous

\section{transluminal angioplasty of}

iliac TASC II A and

$B$ lesions: A randomized

controled trial

LJJ. Bolt ${ }^{1,}$, , AG. Krasznai ${ }^{1, *}$, TA. Sigterman ${ }^{2}$, CJJM. Sikkink ${ }^{1}$, GWH. Schurink ${ }^{3}$. LH. Bouwman ${ }^{1}$ 1. Zuyderland Medical Center, Heerlen, the Netherlands

2. Viecuri Medical Center, Venlo, the Netherlands

3. Maastricht University Medical Center, Maastricht, the Netherlands

${ }^{*}$ LJJ. Bolt and AG. Krasznai are co-first authors

Published in:

Ann Vasc Surg. 2018 Oct 1. pii: S0890-5096(18)30777-5 


\section{Abstract}

Objectives. Percutaneous transluminal angioplasty (PTA) requires the use of nephrotoxic contrast. Patients with chronic kidney disease (CKD) are more prone to develop contrastinduced-nephropathy (CIN) after utilization of contrast. Doppler ultrasound (duplex) guided PTA (DuPTA) is a novel technique and has recently proven to be a successful alternative to conventional PTA in the treatment of iliac stenotic disease, without the use of contrast. In this randomized controlled trial we evaluated whether duplex-guided PTA is as effective as conventional PTA in the treatment of iliac arterial stenotic disease.

Methods. From June 2013 till January 2017, 142 patients with symptomatic peripheral arterial disease (PAD), with significant (>70\%) iliac stenotic lesions (both isolated and patients with multilevel disease without treatment of other lesions) were randomized to receive either conventional PTA or DuPTA of the iliac lesion, regardless of renal function. All patient received an ankle-brachial index (ABI), duplex and MRA in the pre-operative work-up. Primary endpoint was procedural success; defined as passing the guidewire through the stenotic lesion and performing a PTA, with or without stenting. Reduction of peak systolic velocity (PSV) of $\geq 50 \%$ after successful PTA was required in the DUPTA group. Angiographic reduction of more than $50 \%$ was required in the interventional group. Decrease of PSV was evaluated in both groups 4 weeks post-procedural.

Results. Passing of the guidewire through the stenotic lesion was achieved in $96.5 \%$ of the DuPTA-group and $98.8 \%$ in the PTA-group $(\mathrm{p}=0.34)$. Although PSV decreased significantly in both groups 4 weeks post operative, PSV reduction $\geq 50 \%$ was significantly higher in the DuPTA-group, respectively $78 \%$ vs $58 \%$ in the PTA-group ( $<00,01)$. The utilization of stents was significantly greater in the DuPTA-group (resp $52 \%$ vs $18 \%$, $\mathrm{p}<0.01$ ). After correction of potential confounders, significant difference in $\geq 50 \%$ PSV reduction remained; technical success did not significantly differ.

Conclusion. Duplex-guided PTA is a feasible alternative to conventional PTA in the treatment of PAD on the iliac anatomic level. Duplex examination before removal of the guidewire is recommended to evaluate adequate decrease of PSV and identify potential recoil. 


\section{Introduction}

The prevalence of peripheral arterial disease (PAD) is increasing globally, affecting 3-10\% of the population aged $<70$ years and $20 \%$ of the patients $>70$ years. ${ }^{1}$ Due to increasing life expectancy in patients with multiple comorbidities, PAD treatment is often complicated due to chronic diseases such as; chronic kidney disease (CKD), congestive heart failure $(\mathrm{CHF}, \mathrm{EF}<40 \%)$ and diabetes mellitus. Early stage $\mathrm{PAD}$ is often diagnosed due to complaints of cramping of muscles in the lower legs during exercise, which relieves after a short period of rest. This symptom is called intermittent claudication (IC), an early stage symptom of PAD. Supervised exercise therapy (SET) is the preferred treatment in newly diagnosed patients with IC, however when unresponsive to exercise therapy or progression of PAD, either surgical-or endovascular revascularization are treatment of choice. ${ }^{2,3}$ Relative uncomplicated and easily accessible TASC II A and TASC II B lesions are recommended to be treated endovascular, according to the Trans-Atlantic Inter-Society Consensus guidelines (TASCII). ${ }^{1,3,4}$ In common day practice the use of iodine-based contrast in endovascular procedures appears to be unavoidable. The use of iodine contrast is nephrotoxic and can be detrimental to kidney function, especially in patients with known CKD resulting in contrast induced nephropathy $(\mathrm{CIN}){ }^{5,6} \mathrm{CIN}$ following endovascular revascularization is prevalent in $8-15 \%$ of patients regardless of prior renal function. It can increase up to $40-50 \%$ in highrisk patients with multiple comorbidities (chronic kidney disease, diabetes mellitus). ${ }^{7-10}$

Novel therapies have been introduced in the treatment of symptomatic PAD, such as $\mathrm{CO} 2$ mediated percutaneous transluminal angioplasty (PTA) or doppler ultrasound (duplex) guided PTA, to provide an alternative to iodine-based contrast mediated PTA. Recent publications regarding Duplex-guided PTA show that this relative novel technique can be used safely and effectively in the treatment of symptomatic peripheral artery disease (PAD). ${ }^{11-19}$ However, these studies treated femoro-popliteal stenotic lesions. It is argued that duplex in the iliac arteries is complicated due to possible interference of bowel gas and habitus of obese patients. ${ }^{18}$ According to our previous study the feasibility of duplex guided PTA (DUPTA) was shown with a technical success rate of $94 \%$, without any hindrance of duplex visualization. ${ }^{10}$

Currently there are no randomized controlled trials investigating duplex-guided PTA for the treatment of symptomatic PAD. In the current study we performed a RCT with a non-inferiority design, comparing traditional endovascular revascularization with duplexguided PTA in iliac arterial lesions. We hypothesize that duplex-guided PTA is noninferior to conventional PTA regarding technical success in treating iliac lesions TASC II $\mathrm{A}$ and $\mathrm{B}$ with regard to technical success and reduction of PSV. 


\section{Material and Methods}

\section{Study population}

In total 142 patients with PAD diagnosed with significant iliac stenotic lesions ( $>70 \%$ on duplex) were enrolled. After careful evaluation in a multi-disciplinary meeting (vascular surgeons, interventional radiologists and vascular neurophysiologist) all patients with TASC II A and B lesions eligible for PTA were included in this study. Patients were included and treated between June 1, 2013 and February 1, 2017. (Figure 1. Patient flow diagram). 142 patients (81 men [57\%]) were treated for 165 significant iliac lesions. Thirty-four interventions were kissing CIA (common iliac artery) procedures. Only the limb with significant stenotic lesion was included in the analysis. Inclusion criteria were age $\geq 18$ years, diagnosed with Rutherford I-IV not responding to SET, and indication for an elective PTA of a significant iliac stenosis ( $>70 \%$ stenosis), regardless of renal function. Exclusion criteria were iliac occluded lesions (TASC II C and D). Patients with acute limb ischemia were excluded due to the necessity of an acute intervention, whereas a DUPTA demanded presence of two vascular surgeons, required per protocol on approval of the ethics board. Serum creatinin and estimated glomerular filtration rate (eGFR) were obtained within 10 days before procedure to assess renal function. eGFR was calculated using the modified formula of Levey et al. ${ }^{20}$ In accordance to ESUR guidelines (European Society of Urogenital Radiology) no nephrotoxic drugs were administered before procedure. ${ }^{21}$ NSAIDs, diuretics, metformin, ACE-inhibitors, digoxin, lithium, sotalol and colchicine were stopped 24 hours prior to intervention and immediately restarted post-procedure in both groups. The study was performed with approval of the institutional review board (METC Z) and registered at trialregister.nl (NTR4470). All patients provided written informed consent before randomization. 


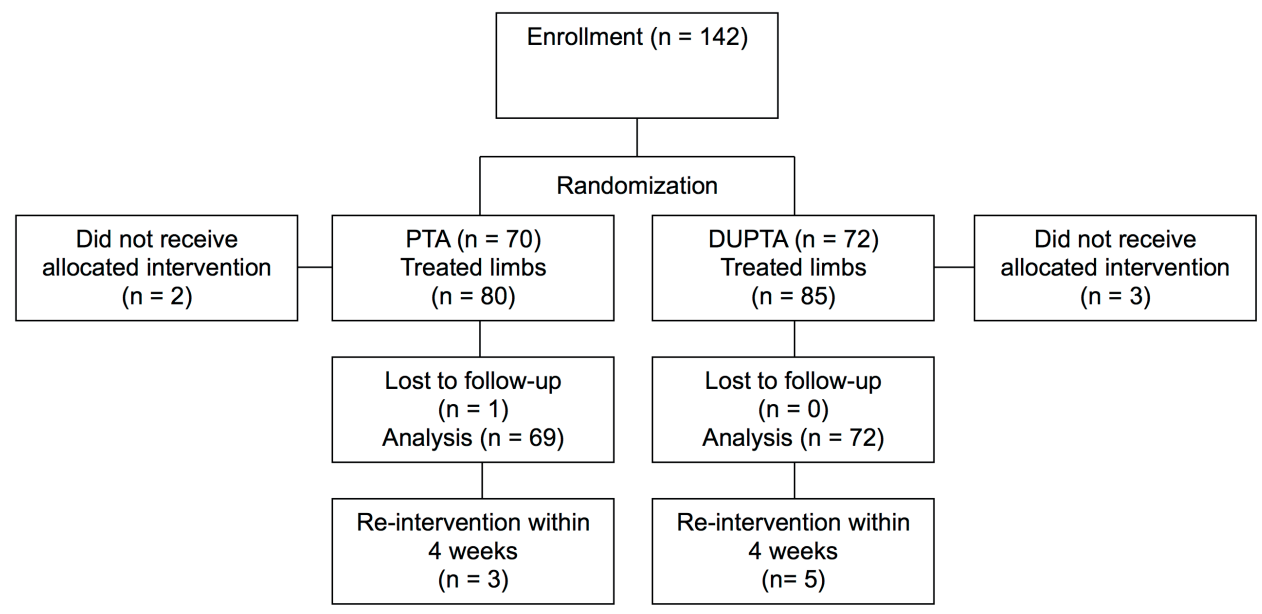

Figure 1. Patient flow diagram. Lost to follow-up within 4 weeks after randomized procedure.

\section{Study protocol}

Patients were randomized in a 1:1 ratio and treated with either duplex-guided PTA (DuPTA-group) or conventional PTA (control group) based on computer-generated random numbers. Preoperative workup of all patients included an ankle brachial index (ABI), arterial mapping with duplex, and magnetic resonance angiography (MRA) of the arterial vessels. Duplex was performed to establish hemodynamic properties of the lesion and whether or not the stenotic lesion was significant and eligible for an endovascular procedure. A 70\% reduction in diameter on duplex (colour flow imaging, B-mode) was considered significant. Blinding of patients and study team was not possible due to difference in intervention procedure. However, vascular technologist evaluating treated stenotic lesion (PSV) four weeks post procedure were blinded to the allocated procedure.

\section{Procedure conventional PTA}

The control group received a conventional PTA of the iliac lesion, utilizing iodine-based contrast and angiography. Patients in the conventional PTA group received one of two contrast agents Omnipaque 240 (GE Healthcare, Pittsburgh, PA) or when known with allergies to contrast or patients requiring hydration, Xenetix 300 (Geurbet, Gorinchem, 
the Netherlands). Patients with eGFR $<45 \mathrm{~mL} / \mathrm{min}$ or with eGFR $<60 \mathrm{~mL} / \mathrm{min}$ and additional risk factors (diabetes mellitus, hypotension (systolic pressure $<90 \mathrm{mmHg}$ ), anaemia (haemoglobin $<6.0 \mathrm{mmol} / \mathrm{L}$ ) and age $>75$ years) for contrast-induced nephropathy received a continuous infusion of isotonic saline for at least $4 \mathrm{~h}$ before and $4 \mathrm{~h}$ after the procedure. High-risk cardiac patients (left ventricular ejection fraction $<40 \%$ ) received $12 \mathrm{~h}$ pre- and post-procedural saline infusion. Conventional PTA procedures were mainly performed under local anesthesia. Post-procedural results of the PTA were evaluated with a completion angiography in two different views to establish success and possible recoil or dissection. The common femoral artery was either closed using a closure devise or manual compression was applied for 10 minutes.

\section{Duplex-guided PTA}

DuPTA-group patients did not receive specific pre- or post-hydration, regardless of renal function. However, all patient received an intra venous line for anaesthetic access with standard infusion of $250 \mathrm{ml} \mathrm{NaCl} 0,9 \%$ per 24 hours. Before final preparation for surgery the stenotic iliac lesion and PSV were measured in the operating theatre utilizing duplex. If the stenotic lesion was occluded after initial diagnosis the procedure was aborted and planned for elective recanalization in the angio-suite. After localization of the iliac stenotic lesion and measurement of the PSV, the patient was prepaired for surgery and received either spinal or general anesthesia. The result after duplex-guided PTA was immediately evaluated utilizing duplex. In case of recoil, dissection or no significant reduction in PSV a stent was placed under duplex guidance. After perceiving a satisfactory result, the procedure was completed. The common femoral artery was either closed using a closure devise or manual compression was applied for 10 minutes.

\section{Procedure Duplex-guided PTA}

Iliac stenosis in the DuPTA-group was visualized perioperative using the latest generation Colour Duplex sonography (Aloka Prosound Alfa7, Aloka Co., Ltd., Tokyo, Japan) with a linear $3.5 \mathrm{MHz}$ probe. Experienced vascular technologists performed the perioperative duplex examination. The vascular technologist took place on the contralateral side of the leg of intervention, opposite of the vascular surgeon.

A sterile plastic cover with covering gel was placed over the linear probe. The common 
femoral artery (CFA) was punctured in a retrograde fashion by the vascular surgeon under ultrasound control ( $5 \mathrm{MHz}$ linear probe). Puncturing of an uncalcified segment of the CFA was achieved without complications in all cases. Insertion of an $11 \mathrm{~cm}, 6$ Fr Brite Tip introducer sheath (Cordis, Cordis Congregation, FL, USA) was performed under Duplex control (guiding the sheath into the external iliac artery). $2500 \mathrm{IU}$ of heparine was intravenously administered to all patients. The artery and stenosis were passed with a Terumo Standard $180 \mathrm{~cm}, 0.035$ guidewire (Terumo, Terumo Europe N.V., Leuven, Belgium) under ultrasound guidance in the majority of subjects. The diameter of the artery and the length of the stenotic segment were measured after localizing the stenosis using B-mode. PSV ratios of the iliac vessels were measured using colour mode. Balloon diameters (Mustang PTA balloon, Boston Scientific, Boston Scientific international S.A., France. Admiral Extreme PTA balloon, Medtronic; Medtronic Luc. Minneapolis, MN, USA), stent sizes and length (Abbott Vascular, Abbot Vascular international BVBA, Diegen, Belgium; Silver PTX drug eluting stent, Cook Medical, Cook Medical Inc., Bloomington, IN, USA; Scuba stent, Medtronic; Omnilink Elite stent) were selected based on stenotic segment measurements utilizing Duplex. Under duplex visualization PTA balloons were inflated (Encore inflation device, Boston, MA, USA) and stents deployed. After deflating and retraction of the balloon, the PSV ratio was measured, leaving the guidewire in position. If PSV ratio was reduced with at least 50\% compared to baseline measurement and no stenosis was evident in B mode the procedure was concluded and deemed successful. To exclude any potential distal embolization the infra-popliteal arterial segment was examined using duplex. Manual compression was applied for at least ten minutes after removal of the endovascular materials. The puncture wound was dressed with compression bandage.

\section{Primary and secondary endpoints}

Primary endpoint was procedural success. Procedural success was defined as passing of the guidewire through the stenotic lesion and performing a PTA, with or without stenting. To conclude the procedure, reduction of peak systolic velocity (PSV) of $\geq 50 \%$ after successful PTA was required in the DUPTA group. Angiographic reduction of more than $50 \%$ was required in the interventional group. Preoperative PSV in the work up was compared to the PSV four weeks post procedure to acquire uniform results without bias. A vascular technologist, blinded to the qualifying procedure, performed duplex four weeks post-procedure. Secondary endpoints were the rate of clinically important 
complications, requiring additional care, between the two interventional groups within 30 days of intervention (flow limiting dissections, post-operative bleeds, infection, seroma), re-intervention within 30 days of intervention and within one year of intervention. Volume of iodine contrast used, radiation dosage area product and fluoroscopy time of every procedure was obtained post procedure.

\section{Follow-up and evaluation}

Control duplex of the treated stenotic lesion, PSV measurements and ABI were performed 4 weeks post-procedure. Thereafter assessment and evaluation of complaints was conducted in the outpatient clinic. The primary endpoint was difference in procedural success between duplex-guided PTA and conventional PTA, defined as 50\% reduction in PSV and successful crossing of the stenotic lesion with a guidewire, dilatation and possible stenting of the lesion. Serum creatinine was evaluated at baseline, 3 days postprocedure, 30 days post-procedure and after one year. eGFR was calculated using the modified formula of Levey et al. ${ }^{20}$

\section{Sample size}

Calculation of the sample size was based on the work of Stuart J. Pocock, ClinicalTrials: A practical approach. ${ }^{22}$ We assumed a procedural success of $88-100 \%$ after conventional iliac angioplasty and an 97\% success rate in duplex-guided iliac PTA's. ${ }^{10,23}$ We assumed the procedural success rate of duplex-guided PTA not to be inferior to conventional PTA, with a clinical acceptable difference of $6 \%$, based on the method for non-inferiority trials. It is customary to assume in the alternative hypothesis that the new method will establish an increase of $6 \%$ in procedural success. With a significance level of 0.05 and $80 \%$ power we calculated a sample size of 71 subjects in each group and a total sample size of 142 to improve the success rate with 6\% in the duplex-guided PTA group.

\section{Statistical analyses}

Categorical variables were presented as percentages and compared using the chi-square test and Fisher's exact in case of low numbers (observed count $<10$, expected count 
$<5)$. Continuous variables were presented as mean \pm standard deviation (SD) and compared using $t$-test for unpaired samples. Missing data were replaced with the mean of 5 imputations. Factorial ANOVA was performed to analyse the effect between the two intervention groups and the use of stents on PSV reduction. Logistic regression was applied to adjust for the difference in PSV pre-procedural. Primary and secondary endpoints were analysed according to the intention-to-treat principle. A p-value $<0.05$ was required for statistical significance, all tests were two-tailed. Analysis is performed according the intention-to-treat principle. Statistical analyses were performed using SPSS software package (IBM Corp. Released 2013. IBM SPSS Statistics for Macintosh, Version 22.0. Armonk, NY: IBM Corp).

\section{Results}

\section{Patient characteristics and procedure results}

A total of 165 stenotic iliac lesions were treated in 142 patients ( 81 men, 61 female, mean age $64.5 \pm 9.3$ years, range $39-88$ years). Seventy patients were treated with conventional PTA, 72 were treated duplex-guided. Body mass index of the patients ranged from 16.5$37 \mathrm{~kg} / \mathrm{m}^{2}$ (mean $25.7 \mathrm{~kg} / \mathrm{m}^{2}$ ). Of the 142 patients, 3 patients received a conventional PTA with contrast due to occlusive disease, whereas at time of diagnosis the stenotic lesions were still patent. All treated lesions were classified as TASC II A and B lesions (resp. 98 and 44) and the vast majority of the patients were classified as Rutherford I and II (resp. 43 and 62). Patients with Rutherford classification I all had focal iliac lesions and did not respond to supervised exercise therapy. No significant differences were present in baseline characteristics between both intervention groups. Chronic kidney disease (CKD), defined as eGFR $<60 \mathrm{ml} / \mathrm{min} / 1.72$ was equally distributed between both groups ( $25 \mathrm{vs} 16$, $\mathrm{p}=0.11$ ). Mean eGFR between the two groups were not significantly different, respectively $75 \mathrm{ml} / \mathrm{min} / 1.73 \mathrm{~m} 2$ in the DuPTA group versus $76 \mathrm{ml} / \mathrm{min} / 1.73 \mathrm{~m}^{2}$ in the PTA group $(\mathrm{p}=0.75)$. Baseline clinical characteristics are presented in Table I. Procedural outcomes are summarized in Table II and III. 
Table 1: Baseline characteristics of study subjects

\begin{tabular}{|c|c|c|c|}
\hline \multirow[t]{2}{*}{ Variables } & \multirow[t]{2}{*}{ DuPTA $(\mathrm{N}=72)$} & \multirow[t]{2}{*}{ PTA $(\mathrm{N}=70)$} & \multirow[b]{2}{*}{ P-value } \\
\hline & & & \\
\hline Gender (male) & 40 & 41 & 0.72 \\
\hline Age (years) & $64[8.3]^{\star}$ & $6_{5}[10.3]$ & 0.82 \\
\hline $\mathrm{BMI}\left(\mathrm{kg} / \mathrm{m}^{2}\right)$ & $25.6[4 \cdot 3]$ & $25 \cdot 9[1.0]$ & 0.62 \\
\hline ASA-classification & & & 0.03 \\
\hline I & 1 & 9 & \\
\hline II & 33 & 34 & \\
\hline III & 37 & 26 & \\
\hline IV & 1 & 0 & \\
\hline Rutherford & & & 0.23 \\
\hline Classification I & 27 & 16 & \\
\hline Classification II & 29 & 33 & \\
\hline Classification III & 15 & 18 & \\
\hline Classification IV & 1 & 3 & \\
\hline TASCII classification & & & 0.58 \\
\hline A & 48 & 50 & \\
\hline B & 24 & 20 & \\
\hline History of smoking & 68 & 67 & 0.44 \\
\hline Duration complaints (months) & $11[10.5]$ & $10[7 \cdot 9]$ & 0.60 \\
\hline History of PTA & 21 & 20 & 0.98 \\
\hline \multicolumn{4}{|l|}{ Co morbidities } \\
\hline Diabetes Mellitus & $5^{8}$ & 55 & 0.77 \\
\hline Familiar vascular disease & 20 & 20 & 0.81 \\
\hline Hypercholesterolemia & 49 & $3^{8}$ & 0.09 \\
\hline Heart Failure $(E F<40)$ & 0 & 2 & 0.15 \\
\hline Hypertension & 42 & 42 & 0.84 \\
\hline Impaired Renal Function (eGFR <6o) & 25 & 16 & 0.11 \\
\hline Creatinine (baseline) & $88[29 \cdot 5]$ & $86[21.1]$ & 0.60 \\
\hline $\operatorname{eGFR}\left(\mathrm{mL} / \mathrm{min} / 1.73 \mathrm{~m}^{2}\right)$ & $75[26.4]$ & 76 [20.9] & 0.75 \\
\hline$>6 o m l$ & 45 & 52 & \\
\hline $45-59 \mathrm{ml}$ & 20 & 12 & \\
\hline $31-45 \mathrm{ml}$ & 4 & 4 & \\
\hline $16-30 \mathrm{ml}$ & 1 & 0 & \\
\hline
\end{tabular}

*Continuous variables are represented as mean with standard deviation. 
Tabel 2: Results

\begin{tabular}{llll}
\hline Variables & DuPTA $(\mathbf{N}=85)$ & PTA $(\mathbf{N}=80)$ & P-value \\
\cline { 2 - 4 } & & & 0.45 \\
\hline Iliac location stenosis & 23 & 27 & \\
CIA & 37 & 32 & \\
EIA $^{\dagger}$ & 9 & 4 & \\
CIA/EIA & 16 & 17 & \\
Kissing CIA & & & 0.16 \\
ABI $^{\ddagger}$ & 0.86 & 0.70 & 0.13 \\
Pre & 0.90 & 0.84 & 0.34 \\
Post & 0.03 & 0.13 & \\
ABI gain & & & 0.02 \\
Peak systolic flow & 488 & 435 & 0.51 \\
$\quad$ Pre & 169 & 177 & $<0.01$ \\
Post & 64 & 55 & $<0.01$ \\
Reduction \% & 66 & 46 & 0.31 \\
50\% reduction & 77 & 75 & 0.34 \\
Degree of stenosis & 82 & 79 & $<0.01$ \\
Technical success & 44 & 14 & 0.52 \\
Stent & 5 & 3 & 0.26 \\
Complication & 8 & 4 & 0.17 \\
Re-intervention within 1 year & 12 & 6 & \\
Re-intervention (total) & & & \\
\hline
\end{tabular}

*CIA (common iliac artery); ${ }^{\dagger}$ EIA (external iliac artery); ${ }^{\ddagger} \mathrm{ABI}$ (ankle-brachial-index)

Tabel 3: Results per subject

\begin{tabular}{llll}
\hline \multirow{2}{*}{ Variables } & DuPTA $(\mathbf{N}=\mathbf{7 2})$ & PTA $(\mathbf{N}=70)$ & \\
\cline { 2 - 4 } & & & P-value \\
\hline Anesthesia & & 52 & $<0.01$ \\
Local & 0 & 8 & \\
Epidural & 38 & 9 & \\
$\quad$ General & 34 & $67.5[33.6]$ & $<0.01$ \\
Contrast (millilitre) & $3.2[12.8]$ & $99.2[133.2]$ & $<0.01$ \\
Radiation dose area product & $4.6[15.1]$ & $6.0[7.0]$ & $<0.01$ \\
Fluoroscopy time (min) & $0.7[2.0]$ & & \\
\hline
\end{tabular}




\section{Primary and secondary endpoints}

Technical success (passing stenotic lesion with guidewire and dilatation of the balloon) was not significantly different between the two interventions. The DuPTA-group showed a success rate of $85 / 82(96.5 \%)$, whereas the conventional PTA-group had an 80/79 $(98.8 \%)$ success rate $(\mathrm{p}=0.34)$. Second primary endpoint, reducing the PSV with $\geq 50 \%$ was achieved in the majority of patients in both groups, however significantly greater decrease was seen in the DuPTA-group (resp. 66 vs 46, $<<0.01$ ). The absolute reduction in PSV was also significantly greater in the DuPTA-group (64\% vs 55\%, p<0.01)(Fig. 1). Furthermore, the utilization of stents was significantly greater in the DuPTA-group (52\% vs $18 \%, \mathrm{p}<0.01)$. Noteworthy, absolute reduction of PSV was greater in patients treated without a stent compared to those treated with stent, although not statistically significant (resp. 61\% vs 56\%, p=0.13). However, when comparing PSV reduction and the use of stents in the separate intervention groups a significant difference was seen in the PTA group (57.3 vs 36.8, $\mathrm{p}<0.01$ ) and not in the DuPTA-group (67.6 vs 62.1), $\mathrm{p}=0.08$ ). Degree of stenotic lesion did not differ between both groups. Degree of stenosis in the DuPTA group was $77 \%$, whereas in the PTA-group the degree of stenosis was $75 \%(\mathrm{p}=0.31)$. Secondary endpoints showed no significant difference between the two interventions. Complication rate in the conventional-PTA group was three, whereas the DuPTA-group had five complications $(\mathrm{p}=0.52)$. None of the complications were correlated with the imaging technique, but rather with the PTA procedure itself. Complications are presented in table IV. No significant difference was seen in the number of re-interventions (DuPTA 5 vs PTA 3, $\mathrm{p}=0.72$ ). None of the patients developed CIN in either interventional group. Furthermore, eGFR was slightly increased three days post intervention, respectively 3.5 $\mathrm{ml} / \mathrm{min} / 1.72$ in the DuPTA-group and $3.2 \mathrm{ml} / \mathrm{min} / 1.72$ in the PTA-group $(\mathrm{p}=0.85)$. Post intervention eGFR after 30days was comparable with baseline values. Slight decrease was seen one year after intervention, however comparable to natural annual renal decline. Within the first year after intervention four patients died, two in each intervention group. None of the mortalities where related to the intervention nor to development of renal failure.

In the DuPTA group x-ray was utilized in 12 patients to aid the guidewire past the stenotic lesion. Eight of these patients received small amounts of iodine contrast (mean $=28.8 \mathrm{ml}$; $3.2 \mathrm{ml}$ in total DuPTA-group), 3 based on per-operative occlusive disease and 5 to assure crural runoff. Volume of contrast used in the PTA-group was significantly greater (67.5 $\mathrm{ml} ; \mathrm{p}<0.01)$. Mean radiation dosage area product in the 12 patients within the DuPTA 
group was $27.7 \mathrm{~Gy}^{*} \mathrm{~cm}^{2}\left(4.6 \mathrm{~Gy}^{*} \mathrm{~cm}^{2}\right.$ in total DuPTA-group), compared to $99.2 \mathrm{~Gy}^{*} \mathrm{~cm}^{2}$ in the PTA group $(\mathrm{p}<0,02)$. Mean fluoroscopy time between the two intervention-groups are not significantly different (when only including the DuPTA patients receiving $\mathrm{x}$-ray $(\mathrm{n}=12)), 4.21$ minutes in the DuPTA-group vs. 5.98 minutes in the PTA-group ( $\mathrm{p}=0.26)$. Average fluoroscopy time in the total DuPTA-group $(\mathrm{n}=72)$ was significantly less compared to the PTA-group (0.70 minutes vs 5.98 minutes, $\mathrm{p}<0.01)$.

\section{Tabel 4: Complications}

\begin{tabular}{ll}
\hline Complications & Intervention group \\
\hline Pseudo aneurysm common femoral artery & DuPTA \\
Luxated atheroma common femoral artery requiring embolectomy & DuPTA \\
Dissection evident zo days post-op, not flow limiting & PTA \\
Dissection + thrombus common femoral artery requiring urokinase treatment & PTA \\
Acute occlusion requiring CERAB procedure & DuPTA \\
Stenosis common femoral artery due to closure device & DuPTA \\
Stenosis common femoral artery due to closure device & PTA \\
Dissection common femoral artery requiring endarteriectomy & DuPTA \\
\hline
\end{tabular}

\section{Discussion}

Endovascular treatment of patients with PAD remains challenging when complicated with chronic kidney disease or iodine related hypersensitivity. The utilization of duplex as an alternative imaging technique in the performance of PTA and even in EVAR procedures has shown to be a good alternative in the treatment of this complicated patient population. ${ }^{11-19,24}$ Given that duplex-guided PTA is viable solution for these patients, we aimed to investigate whether this technique would prove itself non-inferior to conventional PTA in treating iliac lesions.

In this study we showed procedural success of duplex-guided manipulation of a guidewire passing an iliac stenotic lesion non-inferior to conventional PTA. Moreover, the utilization of duplex performing a PTA was superior to conventional PTA with regard to absolute reduction in PSV and the number of procedures reducing the PSV with more than $50 \%$. Beside the greater reduction of PSV in the duplex-guided intervention group, also the numbers stents used was higher in the DuPTA group, which is comparable with the pilot study by Krasznai et al. ${ }^{10}$ Interestingly, the decrease in PSV is less in patients treated with a stent compared to those treated without a stent. This is even significant for 
the PTA group. However, this difference in PSV reduction is not reflected in a decrease of ankle-brachial index or re-interventions. There is no literature available comparing shortterm PSV improvement in patients after treatment of iliac lesions with or without stent. Deloose et al. argues that iliac TASC II A and B lesions should be primary stented, whereas primary patency is $97.4 \%$ after 1 year. ${ }^{25}$ This supports the finding that the difference observed in PSV reduction between the two groups (stent vs no stent) was not clinically relevant. Moreover, PSV at baseline was greater in the intervention group, whereas no significant difference was observed after intervention. Although the PSV value differed at baseline, the degree of stenosis was similar between the two groups. To adjust for PSV at baseline logistic regression analysis was performed. Significant difference in absolute PSV reduction and $>50 \%$ PSV reduction remained. Although PSV reduction was significantly greater in the DuPTA group, no significant difference was seen in $\mathrm{ABI}$ improvement. Even though there was an increase in ABI values, they still remained $<1$. This might be explained by possible distal flow limiting stenotic or occlusive lesions.

Difference in PSV reduction can be explained by the direct hemodynamic feedback after angioplasty with duplex, making it possible to evaluate treatment effect by measuring PSV pre- and post procedure. PSV is an adequate measurement for potential recoil and duplex can also identify hemodynamic significant dissections of the vessel wall after PTA. These attributes are not available with angiography in conventional PTA. Utilization of duplex makes it possible to predict a non successful procedure in $14 \%$ of all interventions, regardless of previous satisfying results on the initial completion angiography. ${ }^{26}$ Completion angiography is only accurate in $76 \%$ of the PTA's, indicating that further dilatation to optimize the vessel lumen is necessary. ${ }^{18}$

The pain experienced during the procedure was not assessed due to the type of anesthesia. Whereas most patients in the control group received local anesthesia, patients in the intervention group received either general- or spinal anesthesia. The protocol of this study was based on a previous feasibility study. ${ }^{10}$ The use of general- or spinal anesthesia relaxes the abdominal muscles and enables the vascular technician to manipulate bowel gases with the probe to stay clear of the area of interest. However, if duplex-guided PTA becomes general practice, there is no need of general- or spinal anesthesia for every straightforward PTA. In the work up of a patient with peripheral arterial disease, all patients receive an arterial duplex of the lower extremities to evaluate degree of stenosis and morphology of the lesion. In none of the study patient's duplex visualization of the aorta-iliac segment was unsuccessful. Furthermore, it is not adamant to have general- or spinal anesthesia 
to perform a duplex-guided iliac PTA. Based on the pre-intervention duplex workup a selection can be made for an advisability of general- or spinal anesthesia.

Serum creatinine and eGFR (estimate glomerular filtration rate) at baseline did not significantly differ between the two groups. None of the patients in either group developed contrast-induced nephropathy (CIN) after the procedure. Serum creatinine observed between the two groups at follow-up 3, 30 days, and 1-year post intervention did not significantly differ between the two groups. These findings are in concurrence with recent findings by Mendes CA et al. studying the effect of carbon dioxide, as an alternative contrast medium, on renal function in patients receiving a PTA of the ilio-femoral segment. The study sample however was very small $(\mathrm{n}=21)$, comparing 11 patients treated with CO2mediated PTA with 10 patients receiving conventional PTA. ${ }^{27}$ No definitive conclusion can be drawn based on these small numbers. Although the sample size of our study is greater, it was not powered to detect a significant difference in serum creatinine increase. With improving visualizing techniques, volume of administered iodine-based contrast is limited and patients with CKD receive pre- and post hydration, limiting nephrotoxic effects of iodine-based contrast.

In the search to reduce the incidence of $\mathrm{CIN}$, carbon dioxide $\left(\mathrm{CO}_{2}\right)$ has been used as an alternative to iodine contrast to perform angiography in high-risk patients with chronic renal disease. ${ }^{27-29}$ Although $\mathrm{CO} 2$ seems a viable solution to substitute iodine contrast, iodine contrast was needed in $21-36 \%$ of the patients to complete the procedure, ${ }^{27,28}$ whereas in our study $11 \%(8 / 72)$ of the patients required contrast. Beside reduced requirement of contrast a great technical advantage of duplex is the possibility to adequately measure the diameter and length of the stenotic lesion. $\mathrm{CO}_{2}$ mediated PTA's or conventional PTA's utilizing contrast will only acquire an image of the intraluminal diameter of the stenotic lesion and both still require the use of $\mathrm{x}$-ray. Furthermore, true vessel diameters can be imaged using ultrasound. This reliable information can be used in the decision, which balloon to use and proper sizing of the stent.

Even though it was not primarily studied in this study. It is the authors believe that duplexguided PTA should be made available for al patients diagnosed with chronic kidney disease and in need of an endovascular intervention of the iliac arteries. Furthermore, a postintervention duplex before removal of the guidewire provides us with direct hemodynamic feedback. Immediate evaluation of procedure result and detection of possible complications could be beneficial for all endovascular procedures. Whereas, recoil of the vessel wall and possible flow limiting dissections can be underestimated on completion angiography. 


\section{Limitations of the study}

This randomized controlled trial was limited by the fact that no blinding could be achieved of the vascular surgeons performing the procedure. Furthermore, patients in the DUPTA group received general anesthesia, whereas patients in the conventional PTA group predominantly received local anesthesia. Patients diagnosed with acute limb ischemia and occluded lesions were not included in the study. Finally, the numbers of patients not meeting inclusion criteria or not willing to participate in the study were not recorded in this study.

\section{Conclusion}

The utilization of duplex to perform a PTA of an iliac stenotic lesion without requirement of iodinated contrast is a valid alternative to conventional PTA with comparable technical success in TASC II A and B lesions. Passing of the stenotic lesion with a guidewire, performing an angioplasty and placement of a balloon expandable stent is feasible and non-inferior to the golden standard, iodine-based contrast mediated PTA. Hemodynamic properties of the treated stenotic lesion should be evaluated using duplex before removal of the guidewire. 


\section{References}

1. Norgren L, Hiatt WR, Dormandy JA, et al. Inter-Society Consensus for the Management of Peripheral Arterial Disease (TASC II). J Vasc Surg. 2007 Jan; 45 Suppl S:S5-67.

2. Wolosker N, Munia MAS, Rosoky R, et al. Surgical treatment for intermittent claudication in patients who do not improve with clinical treatment. Arq Bras Cardiol. 2004 May;82(5):450 4, 445-9.

3. Wolosker N, Nakano L, Rosoky RA, et al. Endovascular treatment for intermittent claudi cation in patients who do not improve with clinical treatment. Clin Sao Paulo Braz. 2005 Jun;60(3):193-200.

4. Wolosker N, Nakano L, Anacleto MMM, et al. Primary utilization of stents in angioplasty of superficial femoral artery. Vasc Endovascular Surg. 2003 Aug;37(4):271-7.

5. Tepel M, Aspelin P, Lameire N. Contrast-induced nephropathy: a clinical and evidence-based approach. Circulation. 2006 Apr 11;113(14):1799-806.

6. Morcos SK, Thomsen HS. Adverse reactions to iodinated contrast media. Eur Radiol. 2001;11(7):1267-75.

7. McCullough PA, Wolyn R, Rocher LL, et al. Acute renal failure after coronary intervention: incidence, risk factors, and relationship to mortality. Am J Med. 1997 Nov;103(5):368-75.

8. Rashid ST, Salman M, Myint F, et al. Prevention of contrast-induced nephropathy in vascular patients undergoing angiography: a randomized controlled trial of intravenous N-acetylcysteine. J Vasc Surg. 2004 Dec;40(6):1136-41.

9. Waybill MM, Waybill PN. Contrast media-induced nephrotoxicity: identification of patients at risk and algorithms for prevention. J Vasc Interv Radiol JVIR. 2001 Jan;12(1):3-9.

10. Krasznai AG, Sigterman TA, Welten RJ, et al. Duplex-guided percutaneous transluminal angioplasty in iliac arterial occlusive disease. Eur J Vasc Endovasc Surg Off J Eur Soc Vasc Surg. 2013 Nov; 46(5):583-7.

11. Ascher E, Marks NA, Schutzer RW, et al. Duplex-guided balloon angioplasty and stenting for femoropopliteal arterial occlusive disease: an alternative in patients with renal insufficiency. $J$ Vasc Surg. 2005 Dec;42(6):1108-13.

12. Ascher E, Marks NA, Hingorani AP, et al. Duplex-guided endovascular treatment for occlusive and stenotic lesions of the femoral-popliteal arterial segment: a comparative study in the first 253 cases. J Vasc Surg. 2006 Dec;44(6):1230-1237-1238.

13. Ascher E, Marks NA, Hingorani AP, et al. Duplex-guided balloon angioplasty and subintimal dissection of infrapopliteal arteries: early results with a new approach to avoid radiation exposure and contrast material. J Vasc Surg. 2005 Dec;42(6):1114-21.

14. Ascher E, Gopal K, Marks N, et al. Duplex-guided endovascular repair of popliteal artery aneurysms (PAAs): a new approach to avert the use of contrast material and radiation exposure. Eur J Vasc Endovasc Surg Off J Eur Soc Vasc Surg. 2010 Jun;39(6):769-73.

15. Marks NA, Hingorani AP, Ascher E. Duplex guided balloon angioplasty of failing infrainguinal bypass grafts. Eur J Vasc Endovasc Surg Off J Eur Soc Vasc Surg. 2006 Aug;32(2):176-81. 
16. Ascher E, Hingorani AP, Marks N. Duplex-guided balloon angioplasty of lower extremity arteries. Perspect Vasc Surg Endovasc Ther. 2007 Mar;19(1):23-31.

17. Ahmadi R, Ugurluoglu A, Schillinger M, et al. Duplex ultrasound-guided femoropopliteal angioplasty: initial and 12-month results from a case controlled study. J Endovasc Ther OffJ Int Soc Endovasc Spec. 2002 Dec;9(6):873-81.

18. Ramaswami G, Al-Kutoubi A, Nicolaides AN, et al. Angioplasty of lower limb arterial stenoses under ultrasound guidance: single-center experience. J Endovasc Surg Off J Int Soc Endovasc Surg. 1999 Feb;6(1):52-8.

19. Kawarada O, Yokoi Y, Takemoto K. Practical use of duplex echo-guided recanalization of chronic total occlusion in the iliac artery. J Vasc Surg. 2010 Aug;52(2):475-8.

20. Levey AS. A New Equation to Estimate Glomerular Filtration Rate. Ann Intern Med. 2009 May 5;150(9):604.

21. Stacul F, van der Molen AJ, Reimer P, et al. Contrast induced nephropathy: updated ESUR Contrast Media Safety Committee guidelines. Eur Radiol. 2011 Dec;21(12):2527- 41.

22. Pocock SJ. Clinical trials: a practical approach. Reprinted. Chichester: Wiley; 2010. 266 p.

23. Kudo T, Chandra FA, Ahn SS. Long-term outcomes and predictors of iliac angioplasty with selective stenting. J Vasc Surg. 2005 Sep;42(3):466-75.

24. Krasznai AG, Sigterman TA, Bouwman LH. Contrast Free Duplex-Assisted EVAR in Patients with Chronic Renal Insufficiency. Ann Vasc Dis. 2014;7(4):426-9.

25. Deloose K, Bosiers M, Callaert J, et al. Primary stenting is nowadays the gold standard treatment for TASC II A \& B iliac lesions: the definitive MISAGO 1-year results. J Cardiovasc Surg (Torino) [Internet]. 2017 Mar [cited 2017 May 16];(3). Available from: http://www. minervamedica.itlindex2.php?show $=R 37 Y 2017 N 03 A 0416$

26. Cluley SR, Brener BJ, Hollier L, et al. Transcutaneous ultrasonography can be used to guide and monitor balloon angioplasty. J Vasc Surg. 1993 Jan;17(1):23-30-31.

27. Mendes C de A, Martins A de A, Teivelis MP, et al. Carbon dioxide contrast medium for endovascular treatment of ilio-femoral occlusive disease. Clin Sao Paulo Braz. 2015 Oct;70(10):675-9.

28. de Almeida Mendes C, de Arruda Martins A, Teivelis MP, et al. Carbon dioxide is a costeffective contrast medium to guide revascularization of TASC A and TASC B femoropopliteal occlusive disease. Ann Vasc Surg. 2014 Aug;28(6):1473-8.

29. Fujihara M, Kawasaki D, Shintani Y, et al. Endovascular therapy by CO2 angiography to prevent contrast-induced nephropathy in patients with chronic kidney disease: a prospective multicenter trial of $\mathrm{CO} 2$ angiography registry. Catheter Cardiovasc Interv Off J Soc Card Angiogr Interv. 2015 Apr;85(5):870-7. 


\section{снартев 6 Renal decline after endovascular intervention for peripheral arterial disease: lodine contrast PTA vs. duplex-guided PTA in TASC II A and B iliac stenotic laesions}

LJJ. Bolt ${ }^{1}$, AG. Krasznai ${ }^{1}$, TA. Sigterman ${ }^{2}$, CJJM. Sikkink ${ }^{1}$, GWH. Schurink ${ }^{3}$. LH. Bouwman ${ }^{1}$ 1. Zuyderland Medical Center, Heerlen, the Netherlands 2. Viecuri Medical Center, Venlo, the Netherlands 3. Maastricht University Medical Center, Maastricht, the Netherlands

Published in: To be submitted 


\section{Abstract}

Background. Endovascular procedures in the management of peripheral arterial disease can induce contrast-induced nephropathy (CIN). However, distinction between CIN and chronic artheroembolic renal disease (AERD) is often difficult. The aim of the present study is to establish influence of guidewire manipulation near renal arteries on postoperative renal function in patients with peripheral arterial disease (PAD) randomized between conventional PTA with iodine contrast and duplex-guided PTA.

Methods. 142 patients with symptomatic PAD caused by iliac stenotic lesions were included between June 2013 and January 2017. After randomization, patients were treated with either conventional PTA, utilizing iodine contrast, or duplex-guided PTA. Serum creatinine and eGFR were determined 10 days prior to intervention. Renal decline was analyzed three day's, one month and 12 months post intervention. Baseline characteristics were acquired from medical records.

Results. eGFR three day's post-intervention increased in both groups, respectively 3,5 $\mathrm{mL} / \mathrm{min}$ in the DuPTA-group and 3,2 $\mathrm{mL} / \mathrm{min}$ in the PTA-group ( $\mathrm{p}=0,85)$. None of the patients developed contrast-induced nephropathy. Alterations of renal function in patients with an eGFR $<60 \mathrm{~mL} / \mathrm{min}$ did not significantly differ between patients treated with DuPTA or PTA (resp. $-5,8$ vs $-5,4 ; \mathrm{p}=1,00$ ). Furthermore, decrease in serum creatinine level post-intervention in patients diagnosed with CKD, is not significantly different compared to patients without CKD (resp. $-5,6$ vs. $-1,9, \mathrm{p}=0,09$ ).

Conclusion. No significant difference in renal decline was observed. Furthermore, none of the patients developed a significant reduction in eGFR. 


\section{Introduction}

Peripheral arterial disease (PAD) is a global problem, affecting over 200 million people and is prevalent in $20 \%$ of the population aged $>70$ years. ${ }^{1,2}$ Symptoms may vary from no limitations in daily activity to major tissue loss of the lower extremities. Upon first presentation patients with PAD are treated with supervised exercise therapy (SET), if SET fails and symptoms remain the same, stenotic lesions are treated either surgically or endovascularly. ${ }^{3,4}$ Less complicated and easy accessible TASC A and B lesions are recommended to be treated with percutaneous transluminal angioplasty (PTA). ${ }^{2,4,5}$ Due to numerous innovations in endovascular techniques the number of endovascular procedures treating PAD have risen exponentially in the last two decades. ${ }^{6}$ Detrimental to this procedure however is the use of nephrotoxic contrast, potentially causing contrastinduced nephropathy (CIN). CIN, defined as an $>25 \%$ increase in serum creatinine or $44 \mathrm{umol} / \mathrm{L}$ within 3 days of intervention, is negligible in patients without chronic kidney disease (CKD), however can be prevalent up to $50 \%$ in high-risk patients with end-stage chronic kidney disease (eGFR $<15 \mathrm{ml} / \mathrm{min} / 1.72){ }^{7-10}$ Chronic kidney disease $(\mathrm{CKD})$ is an increasing global health problem, prevalent in $10-16 \%$ of the world population and up to $45 \%$ in the elderly population (>70years). ${ }^{11}$. Iodine contrast and CKD are both known independent risk factors in the development of CIN. ${ }^{12}$

Incidence of CIN in patients with PAD receiving a contrast mediated endovascular intervention of the lower extremities range from $10-13 \% .^{6,12} \mathrm{CIN}$ is associated with an increased hospitalization time, higher costs and require more additional care. ${ }^{11}$ Up to $0.8 \%$ of the patients require permanent hemodialysis after developing CIN. It is well established that renal impairment might occur after administration of contrast media and is often diagnosed as CIN. However, CIN might be difficult to distinguish from artheroembolic renal disease (AERD). Movement of catheters in femoral arteries and manipulation of guidewires near the renal arteries can dislodge cholesterol debris from atheromatous plaques in the major arteries and cause embolization, inducing mechanical occlusion of the renal arterial bed. ${ }^{13}$

To the best of the authors' knowledge no literature is available establishing incidence in renal deterioration due to catheter manipulation and wire movement near the renal arteries when comparing an endovascular intervention with and without the use of iodine contrast. Prevention of contrast-induced nephropathy can be achieved performing PTA without using nephrotoxic contrast. Duplex-guided PTA does not require the use of nephrotoxic contrast, but is otherwise similar to a regular PTA. This will enable us to study the effect of an endovascular intervention without use of nephrotoxic contrast on renal function. 


\section{Material and Methods}

\section{Study population}

After screening for eligibility in multi disciplinary meetings, we enrolled 142 patients with PAD based on significant iliac stenotic lesions scheduled for PTA. Patients are included and treated between June 1th, 2013 and February 1th, 2017 in a large teaching hospital in the south of the Netherlands, Zuyderland Medical Centre. One hundred and sixty-five significant iliac lesions were treated in 142 patients with PAD (81 men [57\%]). Included patients were aged $\geq 18$ years and diagnosed with a significant iliac stenotic lesion ( $>70 \%$ lumen loss), not responding to supervised exercise therapy (SET) and requiring an elective PTA. Patients were included regardless of renal function. Patients were excluded when iliac arteries were totally occluded or when duplex visualization of the lesion was not possible due to bowel gas or extreme obesity. Renal function was assessed measuring serum creatinine and estimated glomerular filtration rate (eGFR) within 10 days before procedure. eGFR is was calculated using the modified formula of Levey et al. ${ }^{14}$ Reno protective drugs (NSAIDs, diuretics, metformin, ACE-inhibitors, digoxin, lithium, sotalol and colchicine) were stopped 24 hours prior to intervention and immediately restarted after the procedure, in accordance with the national renal protective program.

\section{Study protocol, Follow-up and evaluation}

Randomization was performed using computer-generated random numbers in a $1: 1$ ratio, assigning treatment to either conventional PTA (control-group) or duplex-guided PTA (DuPTA-group). The control-group received a conventional PTA of the iliac lesion, utilizing iodinated contrast and $\mathrm{x}$-ray. Whereas patients in the DuPTA-group received a PTA under duplex-guidance. Serum creatinine and estimated glomerular filtration rate (eGFR) were evaluated at baseline, within 10 day before intervention. Follow-up regarding renal function occurred 3-5 day's and 30 day's post-procedure. When available creatinine and glomerular filtration rate were acquired 1 year before randomization and 1 year post-intervention. Beside renal function, patency of the treated stenotic lesion and clinical symptoms were evaluated. 


\section{Endovascular procedure}

Conventional PTA's were performed by one of three experienced vascular surgeons or by one of two staff interventional radiologists in an endovascular suite equipped with a Philips Allura Xper FD20 (Phillips, Eindhoven, The Netherlands). Whereas all duplex-guided PTA's were performed by two vascular surgeons in a hybrid operating room (OR). Patients in the conventional PTA group received one of two contrast agents, either Omnipaque 240 (GE Healthcare, Pittsburgh, PA) or when known with allergies to contrast agents, Xenetix 300 (Geurbet, Gorinchem, the Netherlands). Patients with eGFR $<45 \mathrm{~mL} / \mathrm{min}$ or with eGFR $<60 \mathrm{~mL} / \mathrm{min}$ and additional risk factors (diabetes mellitus, heart failure, hypotension, anemia, and age $>75$ years) for contrast-induced nephropathy received a continues infusion of isotonic saline for at least $4 \mathrm{~h}$ before and $4 \mathrm{~h}$ after the procedure. High-risk cardiac patients (left ventricular ejection fraction $<40 \%$ ) received $12 \mathrm{~h}$ pre- and post-procedural saline infusion. DuPTA-group patients did not receive pre- or posthydration.

\section{Risk factor definitions}

Heart failure was defined as decreased left ventricular ejection fraction of maximal 40\%. ${ }^{15}$ Diabetes mellitus was defined as the requirement of hypoglycemic drugs or fasting blood glucose levels of $>120 \mathrm{mg} / \mathrm{dL}$ or glycosylated hemoglobin $>6 \%{ }^{16,17}$ Hyperlipidemia was defined as the use of lipid-lowering drug or presence of total serum cholesterol level $>5$ $\mathrm{mmol} / \mathrm{L}$, high-density lipoprotein cholesterol level $<1 \mathrm{mmol} / \mathrm{L}$, or triglyceride level $>2$ $\mathrm{mmol} / \mathrm{L} .{ }^{16,17}$ Furthermore, hypertension was defined as the use of any antihypertensive drug or measurement of systolic blood pressure $>140 \mathrm{mmHg}$ and/or diastolic blood pressure of $>90 \mathrm{mmHg}$ on two separate occasions. ${ }^{16,17}$ Smoking status was scored positive if the patient was an active smoker or had a history of smoking. Definition of hereditary cardiovascular disease was characterized as a history of cardiovascular events in first-degree relatives.

\section{Sample size}

Thestudywaspowered toestablish proceduralsuccessofaduplex-guidedPTA. Thesamplesizeis not powered to significantly differentiate in incidence ofCIN between conventional PTA with contrast and duplex-guided PTA. A total of 142 patients are treated with 165 stenotic lesions. 
The study was performed with approval of the institutional review board and is in accordance with the declaration of Helsinki. All patients provided written informed consent before randomization.

\section{Statistical analyses}

Categorical variables are presented as percentages and were compared using the chi-square test and Fisher's exact in case of low numbers (observed count $<10$, expected count $<5$ ). Continues variables are presented as mean \pm standard deviation (SD) and compared using the $t$-test for unpaired samples. Missing data were replaced with the mean of 5 imputations. Log linear analysis was performed to adjust for the incidence of chronic renal failure between both interventions, whereas logistic regression was applied to adjust for the difference in peak systolic velocity (PSV) pre-procedure. Primary and secondary endpoint were analysed according the intention-to-treat principle. A p-value $<0.05$ was required for statistical significance, all tests were two-tailed. Statistical analysis was performed using SPSS software package (IBM Corp. Released 2013. IBM SPSS Statistics for Macintosh, Version 22.0. Armonk, NY: IBM Corp).

\section{Results}

A total of 142 patients are included in this randomized controlled trial, of which 72 patients are treated with a duplex-guided PTA and 70 patients with conventional PTA. Average age of the study population ranged from 39 to 88 years (mean 65). Mean follow-up is 10 months (range 0-36 months). Patients were predominantly diagnosed with peripheral artery disease, Rutherford classification I and II (resp. 30\% and 44\%). Treated lesions were all TASC II A and B lesions (resp. 69\% and 31\%). Baseline renal function did not significantly differ between the two interventions; eGFR in the DuPTA-group was $75 \mathrm{~mL} / \mathrm{min} / 13.73 \mathrm{~m}^{2}$ and $76 \mathrm{~mL} / \mathrm{min} / 13.73 \mathrm{~m}^{2}$ in the conventional PTA-group. Furthermore, number of patients with chronic kidney disease was not significantly different, respectively 25 patients in the DuPTA-group versus 16 patients in the conventional PTA-group $(\mathrm{p}=0.11)$. Concomitant risk factors included diabetes mellitus (80\%), hypercholesterolemia (61\%), hypertension (59\%), congestive heart failure (1,4\%), familiar vascular disease $(39 \%)$, and history of smoking (96\%). Risk factors did not significantly differ between the two groups. Ninety-six percent of the patients received statin therapy after diagnosis of $\mathrm{PAD}$ and al patients received oral anticoagulation ( $97 \%$ antiplatelet). Baseline characteristics are summarized in table I. 
Table 1: Baseline characteristics of study subjects

\begin{tabular}{|c|c|c|c|}
\hline \multirow[t]{2}{*}{ Variables } & \multirow[t]{2}{*}{ DuPTA $(\mathrm{N}=72)$} & \multicolumn{2}{|l|}{ PTA $(\mathrm{N}=70)$} \\
\hline & & & P-value \\
\hline Gender (male) & 40 & 41 & 0.72 \\
\hline Age (years) & $64[8.3]$ & $65[10.3]$ & 0.82 \\
\hline BMI $\left(\mathrm{kg} / \mathrm{m}^{2}\right)$ & $25.6[4 \cdot 3]$ & $25.9[1.0]$ & 0.62 \\
\hline ASA-classification & & & 0.03 \\
\hline 1 & 1 & 9 & \\
\hline II & 33 & 34 & \\
\hline III & 37 & 26 & \\
\hline IV & 1 & o & \\
\hline Rutherford & & & 0.51 \\
\hline Classification I & 26 & 19 & \\
\hline Classification II & 29 & 29 & \\
\hline Classification III & 18 & 21 & \\
\hline Classification IV & 1 & 3 & \\
\hline TASCII classification & & & 0.58 \\
\hline A & 48 & 49 & \\
\hline B & 24 & 20 & \\
\hline History of smoking & 68 & 67 & 0.44 \\
\hline Duration complaints (months) & $11[10.5]$ & $10[7.9]$ & 0.60 \\
\hline History of PTA & 21 & 20 & 0.98 \\
\hline \multicolumn{4}{|l|}{ Co morbidities } \\
\hline Diabetes Mellitus & 58 & 55 & 0.77 \\
\hline Familiar vascular disease & 20 & 20 & 0.81 \\
\hline Hypercholesterolemia & 49 & 38 & 0.09 \\
\hline Heart Failure $(E F<40)$ & o & 2 & 0.15 \\
\hline Hypertension & 42 & 42 & 0.84 \\
\hline Impaired Renal Function $(\mathrm{eGFR}<60$ ) & 25 & 16 & 0.11 \\
\hline Creatinine (baseline) & $88[29.5]$ & $86[21.1]$ & 0.60 \\
\hline eGFR $\left(\mathrm{mL} / \mathrm{min} / 1.73 \mathrm{~m}^{2}\right)$ & $75[26.4]$ & $76[20.9]$ & 0.75 \\
\hline$>6 o m l$ & 45 & 52 & \\
\hline $45-59 \mathrm{ml}$ & 20 & 12 & \\
\hline $31-45 \mathrm{ml}$ & 4 & 4 & \\
\hline $16-30 \mathrm{ml}$ & 1 & o & \\
\hline
\end{tabular}


In both study-groups rise of eGFR was observed three/five day's post-intervention, 3,5 $\mathrm{mL} / \mathrm{min}$ in the DuPTA-group and $3,2 \mathrm{~mL} / \mathrm{min}$ in the conventional PTA-group $(\mathrm{p}=0,85)$. None of the patients from either intervention group developed contrast-induced nephropathy. Renal function 30 day's post-intervention was also improved in both studygroups, respectively $1.20 \mathrm{~mL} / \mathrm{min}$ in the DuPTA-group and $1.55 \mathrm{~mL} / \mathrm{min}$ in the PTAgroup $(\mathrm{p}=0,84)$. Seventy-one patients had a valid eGFR one year post-intervention (11-13 months). A greater decrease of eGFR was seen in the DuPTA-group $(-2,68)$ compared to the PTA-group $(-0,64)$, however not significant $(\mathrm{p}=0,47)$. Furthermore, $48 \%$ of the patients receiving the duplex-guided intervention showed fast renal decline, compared to $30 \%$ receiving a conventional PTA $(\mathrm{p}=0,13)$. Fast renal decline is defined as $>4 \mathrm{ml} / \mathrm{min}$ loss of eGFR within one year. ${ }^{17}$

Factorial ANOVA showed no significant difference in rise of serum creatinine three/ five day's post-intervention in patients with an eGFR $<60 \mathrm{~mL} / \mathrm{min}$ comparing patients treated with either DuPTA or PTA (resp. $-5,8$ vs $-5,4 ; \mathrm{p}=1,00$ ). Furthermore, patients diagnosed with chronic kidney injury, decrease in serum creatinine level post-intervention is not significantly different to patients without CKD (resp. $-5,6$ vs. $-1,9, \mathrm{p}=0,09$ ). Results regarding renal function are summarized in table 2 .

Tabel 2: Renal function

\begin{tabular}{llll}
\hline Variables & DuPTA $(\mathbf{N}=\mathbf{7 2})$ & PTA $(\mathbf{N}=\mathbf{7 0})$ & \\
\cline { 2 - 4 } & & & P-value \\
\hline Creatinine & 86 & 87 & 0.86 \\
Pre 1 year & 88 & 86 & 0.60 \\
Pre & 86 & 83 & 0.59 \\
Post 3 days & 88 & 86 & 0.81 \\
Post 30 days & 89 & 91 & 0.71 \\
1 year post & & & \\
Rise Creatinine & -3.6 & -2.5 & 0.57 \\
Day 3 & -0.27 & -0.75 & 0.76 \\
Day 30 & 1.36 & 0.93 & 0.87 \\
Year 1 & & & \\
eGFR & 74 & 76 & 0.71 \\
Pre 1 year & 75 & 76 & 0.75 \\
Pre & 77 & 80 & 0.54 \\
Post 3 days & 77 & 76 & 0.86 \\
Post 30 days & 74 & 70 & 0.50 \\
1 year post & & & \\
Rise eGFR & 3.5 & 3.2 & 0.85 \\
Day 3 & 1.20 & 1.55 & 0.84 \\
Day 30 & -2.68 & -0.64 & 0.47 \\
Year 1 & & & \\
\hline
\end{tabular}


Within the DuPTA-group eight patients were exposed to a small dose of iodine contrast (mean $=28,8 \mathrm{ml} \pm 28 \mathrm{ml}$ ). Due to progression of PAD, three patients had an occlusion at time of intervention, whereas at time of diagnosis the lesions were still patent. Five patients received contrast to evaluate the result after a duplex-guided intervention. However, within the PTA-group volume of contrast administered was significantly greater (mean 67,5 $\mathrm{ml} \pm 34 \mathrm{ml} ; \mathrm{p}<0,01$ ). Twelve patients in the DuPTA-group received $\mathrm{x}$-ray radiation to aid the guidewire past the stenotic lesion, with a mean radiation dosage area product of 27,71 $\mathrm{Gy}^{*} \mathrm{~cm}^{2}$. Patients in the PTA-group received a mean radiation dosage area product of $99,18 \mathrm{~Gy}^{*} \mathrm{~cm}^{2}(\mathrm{p}=0,02)$. In those DuPTA patients where $\mathrm{x}$-ray was utilized fluoroscopy time was comparable to PTA patients (resp. 4,2 minutes vs 6,0 minutes; $\mathrm{p}=0,26$ ). Procedure characteristics are summarized in table 3 .

In total four patients died (within one year of intervention), two in each intervention group. None of the mortalities were related to peripheral artery disease or intervention.

Tabel 3: Procedure characteristics per subject

\begin{tabular}{llll}
\hline \multirow{2}{*}{ Variables } & DuPTA $(\mathbf{N}=\mathbf{7 2})$ & PTA $(\mathbf{N}=70)$ & \\
\cline { 2 - 4 } & & & P-value \\
\hline Contrast (millilitre) & $3.2[12.8]$ & $67.5[33.6]$ & $<0.01$ \\
Radiation dose area product & $4.6[15.1]$ & $99.2[133.2]$ & $<0.01$ \\
Fluoroscopy time & $0.7[2.0]$ & $6.0[7.0]$ & $<0.01$ \\
\hline
\end{tabular}

\section{Discussion}

The aim of the present study was to determine whether the incidence of CIN would decline if an endovascular intervention were to be performed without the utilization of iodine contrast and evaluate the role of guidewire manipulation on renal function. Duplexguided PTA enabled us to treat stenotic iliac lesions, with or without stenting, not having to use nephrotoxic contrast. Beside difference in imaging technique the procedure did not differ from a conventional PTA. This technique offered us the possibility to evaluate the effect of a PTA procedure on renal function without being confounded by possible contrast induced nephropathy. 
CIN is a well-known complication of endovascular diagnostic and therapeutic interventions requiring the utilization of iodine contrast. Although often of nonsignificance in a patient with a healthy renal function, patients with chronic kidney disease $(\mathrm{CKD})$ might experience severe complications after developing CIN with high morbidity and mortality. ${ }^{18} \mathrm{~A}$ large retrospective study $(\mathrm{n}>16000)$ undergoing a procedure requiring contrast showed a 5,5-fold increased risk of death. ${ }^{19}$ Regrettably, due to the progressive nature of atherosclerosis and perseverance in risk behavior, patients often require multiple angiograms and endovascular interventions in the course of their disease. Increased exposure to iodine contrast required in diagnostic and therapeutic procedures, increases incidence and prevalence of CIN annually with 5-8\%. ${ }^{20}$. Remarkably, the current article did not identify a single patient with CIN post-intervention and no mortality within one year related to CKD. Whereas a recent study by Krasznai et al. described an incidence of $13 \%$ in patients treated with an endovascular intervention for symptomatic peripheral arterial disease. Although the number of endovascular interventions increase globally, the overall incidence of CIN decreases in cardiac patients from $15 \%$ to $7 \% .{ }^{18}$ Due to greater awareness, improved preventive measures and decreased nephrotoxicity of newly developed contrast media, great strides are made. However, the absolute number of patients diagnosed with CIN remains high due to the ever-increasing number of endovascular procedures.

\section{$A E R D$}

Renal impairment after utilization of contrast in endovascular procedures does not consequently mean that the damage inflicted is solely caused by the nephrotoxic properties of iodine contrast. Manipulation of catheters and guidewires might induce intravascular trauma by dislodging atheromatous plaques in the suprarenal aorta and consequently occlude renal arteries, arterioles and/or glomerular capillaries with cholesterol crystals. ${ }^{21}$ Keeley et al. observed plaque debris on guiding catheters after femoral approach to perform percutaneous revascularization in $>50 \%$ of the study population $(n=1000){ }^{22}$ However, incidence of artheroembolic renal disease (AERD) remains uncertain. Diagnosis is frequently acquired in post-mortem autopsy. Arteroembolic renal disease is most often iatrogenic and accounts for $76-77 \%$ of al diagnosed cases of AERD. ${ }^{23-27}$ Of the iatrogenic causes $80 \%$ is induced due to angiographic interventions, such as percutaneous 
transluminal angioplasty. $(23,26,28)$ Distinction should be made between acute, sub-acute and chronic progression of renal function decline. Whereas clinical course of acute and sub-acute arteroembolic renal disease can be quite severe, requiring dialysis in 20-61\%, chronic progression can be quite mild and can go completely unrecognized. ${ }^{23-25,28}$ Even though chronic arteroembolic renal disease can manifest undetected, it is still progressive and glomerular filtration rate will decline gradually. Sigterman et al. described a long-term loss in renal function after endovascular procedures in patients with symptomatic PAD. In this study by Sigterman et al. patients, diagnosed with Rutherford class II to class VI, were all treated endovascularly. ${ }^{12}$ Patients developing CIN had a significant greater reduction in eGFR after one year, compared to patients without CIN. Still patients without CIN had a decline of $6.2 \mathrm{ml} / \mathrm{min} / 1.73 \mathrm{~m}^{2}$ after one year, whereas normal renal decline is estimated to be $0.4 \pm 3.6 \mathrm{ml} / \mathrm{min} / 1.73 \mathrm{~m}^{2}$ in elderly people. ${ }^{29}$ In our study none of the patients developed CIN and annual decline of renal function in the patients treated with a conventional PTA was $0,64 \mathrm{ml} / \mathrm{min} / 1.73 \mathrm{~m}^{2}$, which is comparable to natural annual decline. Renal decline was analysed and CIN was diagnosed according to KDIGO guidelines. ${ }^{8}$ However, making the distinction between CIN and AERD is difficult. The fact that Sigterman et al, and al studies alike, did not performed a renal biopsy makes it difficult to differentiate between the two diagnoses. It could well be possible that some patients experienced a combination of $\mathrm{CIN}$ and chronic arteroembolic renal diseases or only chronic arteroembolic renal disease. Risk factors for the development of chronic arteroembolic renal diseases are similar to patients with PAD. Mainly male patients aged $>60$ years, history of tobacco use, diagnosed with hypertension and/or diabetes mellitus and renal failure prior to intervention are at risk. To differentiate between AERD and CIN the timeframe in which serum creatinine rises is key. Whereas CIN shows a rise in serum creatinine within 24 hours post-procedure and peaks at 5 days post-procedure, AERD shows a more gradual rise in serum creatinine and may take weeks to be diagnosed. ${ }^{21,30}$ Moreover, severe cases of AERD will show clinic signs such as skin lesions (blue to syndrome, livedo reticularis). ${ }^{21}$ Long term effects on renal function after developing AERD can vary between spontaneous resolution and requirement of dialysis due to end-stage renal disease. ${ }^{31}$

There is no specific treatment for AERD. However, when diagnosed with AERD supportive treatment for renal failure should be initiated as well as preventive measurements for potential reoccurrence. Limited evidence is available suggesting statin therapy stabilizes plaques and minimalize future cholesterol embolization. ${ }^{31}$ Traditional risk factors should be modified, most importantly cessation of smoking and management of hypertension and serum cholesterol. 


\section{Conclusion}

The incidence of CIN in patients treated endovascularly for TASCII A and B iliac lesions do not significantly differ between patient treated with or without iodine contrast. No conclusion can be made regarding etiology of renal decline post intervention distinguishing between CIN and AERD. Adequately powered studies are required to differentiate between CIN and AERD in patients treated for PAD with an endovascular procedure of the lower extremities. 


\section{References}

1. Fowkes FGR, Rudan D, Rudan I, Aboyans V, Denenberg JO, McDermott MM, et al. Comparison of global estimates of prevalence and risk factors for peripheral artery disease in 2000 and 2010: a systematic review and analysis. Lancet Lond Engl. 2013 Oct 19;382(9901):1329-40.

2. Norgren L, Hiatt WR, Dormandy JA, Nehler MR, Harris KA, Fowkes FGR, et al. InterSociety Consensus for the Management of Peripheral Arterial Disease (TASC II). J Vasc Surg. 2007 Jan; 45 Suppl S:S5-67.

3. Wolosker N, Munia MAS, Rosoky R, Fidelis RJ, Nakano L, Kauffman P, et al. Surgical treatment for intermittent claudication in patients who do not improve with clinical treatment. Arq Bras Cardiol. 2004 May;82(5):450-4, 445-9.

4. Wolosker N, Nakano L, Rosoky RA, Munia MA, Netto BM, Puech-Leão P. Endovascular treatment for intermittent claudication in patients who do not improve with clinical treatment. Clin Sao Paulo Braz. 2005 Jun;60(3):193-200.

5. Wolosker N, Nakano L, Anacleto MMM, Puech-Leão P. Primary utilization of stents in angioplasty of superficial femoral artery. Vasc Endovascular Surg. 2003 Aug;37(4):271-7.

6. Prasad A, Ortiz-Lopez C, Khan A, Levin D, Kaye DM. Acute kidney injury following peripheral angiography and endovascular therapy: A systematic review of the literature. Catheter Cardiovasc Interv Off J Soc Card Angiogr Interv. 2016 Aug;88(2):264-73.

7. Neyra JA, Shah S, Mooney R, Jacobsen G, Yee J, Novak JE. Contrast-induced acute kidney injury following coronary angiography: a cohort study of hospitalized patients with or without chronic kidney disease. Nephrol Dial Transplant Off Publ Eur Dial Transpl Assoc - Eur Ren Assoc. 2013 Jun;28(6):1463-71.

8. Ad-hoc working group of ERBP, Fliser D, Laville M, Covic A, Fouque D, Vanholder R, et al. A European Renal Best Practice (ERBP) position statement on the Kidney Disease Improving Global Outcomes (KDIGO) clinical practice guidelines on acute kidney injury: part 1: definitions, conservative management and contrast-induced nephropathy. Nephrol Dial Transplant Off Publ Eur Dial Transpl Assoc - Eur Ren Assoc. 2012 Dec;27(12):4263-72.

9. McCullough PA, Wolyn R, Rocher LL, Levin RN, O’Neill WW. Acute renal failure after coronary intervention: incidence, risk factors, and relationship to mortality. Am J Med. 1997 Nov;103(5):368-75.

10. Waybill MM, Waybill PN. Contrast media-induced nephrotoxicity: identification of patients at risk and algorithms for prevention. J Vasc Interv Radiol JVIR. 2001 Jan;12(1):3-9.

11. Coresh J, Selvin E, Stevens LA, Manzi J, Kusek JW, Eggers P, et al. Prevalence of chronic kidney disease in the United States. JAMA. 2007 Nov 7;298(17):2038-47.

12. Sigterman TA, Krasznai AG, Snoeijs MG, Heijboer R, Schurink GWH, Bouwman LH. Contrast Induced Nephropathy and Long-term Renal Decline After Percutaneous Transluminal Angioplasty for Symptomatic Peripheral Arterial Disease. Eur J Vasc Endovasc Surg Off J Eur 
Soc Vasc Surg. 2015 Oct 9;

13. Stratta P, Bozzola C, Quaglia M. Pitfall in nephrology: contrast nephropathy has to be differentiated from renal damage due to atheroembolic disease. JNephrol. 2012 Jun;25(3):2829 .

14. Levey AS. A New Equation to Estimate Glomerular Filtration Rate. Ann Intern Med. 2009 May 5;150(9):604.

15. American Heart Association. Ejection Fraction Heart Failure Measurement. American Heart Association; 2015.

16. Diehm N, Shang A, Silvestro A, Do D-D, Dick F, Schmidli J, et al. Association of cardiovascular risk factors with pattern of lower limb atherosclerosis in 2659 patients undergoing angioplasty. Eur J Vasc Endovasc Surg OffJ Eur Soc Vasc Surg. 2006 Jan;31(1):59-63.

17. Sigterman TA, Krasznai AG, Snoeijs MG, Heijboer R, Schurink GWH, Bouwman LH. Contrast Induced Nephropathy and Long-term Renal Decline After Percutaneous Transluminal Angioplasty for Symptomatic Peripheral Arterial Disease. Eur J Vasc Endovasc Surg Off J Eur Soc Vasc Surg. 2016 Mar;51(3):386-93.

18. McCullough PA, Adam A, Becker CR, Davidson C, Lameire N, Stacul F, et al. Epidemiology and prognostic implications of contrast-induced nephropathy. Am J Cardiol. 2006 Sep 18;98(6A):5K-13K.

19. Levy EM, Viscoli CM, Horwitz RI. The effect of acute renal failure on mortality. A cohort analysis. JAMA. 1996 May 15;275(19):1489-94.

20. Parfrey PS, Griffiths SM, Barrett BJ, Paul MD, Genge M, Withers J, et al. Contrast materialinduced renal failure in patients with diabetes mellitus, renal insufficiency, or both. A prospective controlled study. $N$ Engl J Med. 1989 Jan 19;320(3):143-9.

21. Scolari F, Ravani P. Atheroembolic renal disease. Lancet LondEngl. 2010 May 8;375(9726):1650 60.

22. Keeley EC, Grines CL. Scraping of aortic debris by coronary guiding catheters: a prospective evaluation of 1,000 cases. J Am Coll Cardiol. 1998 Dec;32(7):1861-5.

23. Belenfant X, Meyrier A, Jacquot C. Supportive treatment improves survival in multivisceral cholesterol crystal embolism. Am J Kidney Dis Off J Natl Kidney Found. 1999 May;33(5):840 50.

24. Scolari F, Tardanico R, Zani R, Pola A, Viola BF, Movilli E, et al. Cholesterol crystal embolism: A recognizable cause of renal disease. Am J Kidney Dis Off J Natl Kidney Found. 2000 Dec;36(6):1089-109.

25. Scolari F, Ravani P, Pola A, Guerini S, Zubani R, Movilli E, et al. Predictors of renal and patient outcomes in atheroembolic renal disease: a prospective study. J Am Soc Nephrol JASN. 2003 Jun;14(6):1584-90.

26. Scolari F, Ravani P, Gaggi R, Santostefano M, Rollino C, Stabellini N, et al. The challenge of diagnosing atheroembolic renal disease: clinical features and prognostic factors. Circulation. 2007 Jul 17;116(3):298-304.

27. Mittal BV, Alexander MP, Rennke HG, Singh AK. Atheroembolic renal disease: a silent 
masquerader. Kidney Int. 2008 Jan;73(1):126-30.

28. Thadhani RI, Camargo CA, Xavier RJ, Fang LS, Bazari H. Atheroembolic renal failure after invasive procedures. Natural history based on 52 histologically proven cases. Medicine (Baltimore). 1995 Nov;74(6):350-8.

29. Shlipak MG, Katz R, Kestenbaum B, Fried LF, Newman AB, Siscovick DS, et al. Rate of Kidney Function Decline in Older Adults: A Comparison Using Creatinine and Cystatin C. Am J Nephrol. 2009;30(3):171-8.

30. Frock J, Bierman M, Hammeke M, Reyes A. Atheroembolic renal disease: experience with 22 patients. Nebr Med J. 1994 Sep;79(9):317-21.

31. Saric M, Kronzon I. Cholesterol embolization syndrome. Curr Opin Cardiol. 2011 Nov;26(6):472-9. 


\section{part II Possible solution \\ preventing postcontrast \\ acute kidney injury}




\section{снартев Prevention of postcontrast acute kidney injury after percutaneous transluminal angioplasty by inducing RenalGuard controlled furosemide forced diuresis with matched hydration: study protocol for a randomised controlled trial}

LJJ. Bolt ${ }^{1}$, TA. Sigterman ${ }^{2}$, AG. Krasznai ${ }^{1}$, CJJM. Sikkink ${ }^{1}$, GWH. Schurink ${ }^{3}$, LH. Bouwman ${ }^{1}$ 1. Zuyderland Medical Centre, Heerlen, the Netherlands 2. Viecuri Medical Centre, Venlo, the Netherlands 3. Maastricht University Medical Centre, Maastricht, the Netherlands

Published in:

BMJ Open. 2018 Oct 4;8(9):e021842 


\section{Abstract}

Introduction. Percutaneous transluminal interventions (PTA) are often complicated due to post contrast acute kidney injury (PC-AKI) in patients diagnosed with chronic kidney disease (CKD). Hydration therapy is the cornerstone in the prevention of PCAKI. Furosemide forced diuresis with matched hydration using the Renalguard system ${ }^{\mathrm{TM}}$ enables a steady balance between diuresis and hydration. A randomized controlled trial will be performed in order to investigate whether furosemide forced diuresis with matched hydration in combination with the Renalguard system ${ }^{\mathrm{TM}}$ decreases incidence of PC-AKI in patients with CKD receiving a PTA of the lower extremities. Furthermore, we will investigate whether sampling of urine biomarkers 4 hours after intervention can detect PC-AKI in an earlier stage compared to the golden standard, serum creatinine 48-72 hours post intervention.

Methods and analysis. a single centre randomized controlled trial will be conducted. Patients $>18$ years in need of a PTA of the lower extremities and diagnosed with CKD will be randomly assigned to receive either standard of care pre- and post-hydration or furosemide forced diuresis with matched hydration periprocedural using the Renalguard system $^{\mathrm{TM}}$. Four hours post intervention a urine sample will be collected of all participating patients. Serum creatinine will be sampled within 10 days prior to intervention as well as 1, 3 and 30 days post intervention. The primary endpoint is incidence of PC-AKI post PTA. Secondary endpoint is the rise of urine biomarkers 4 hours post intervention.

Ethics and dissemination. Study protocol is approved by the research ethics committee and institutional review board (reference number 16T-201 and NL59809.096.16). Study results will be disseminated by oral presentation at conferences and will be submitted to a peer-reviewed journal. It is anticipated that study results will offer a solution to contrast induced nephropathy in patients with chronic kidney disease receiving a PTA of the lower extremities.

Trial registration number: NTR6236

EudraCT number: 2016-005072-10 


\section{Introduction}

\section{Background}

Endovascular treatment of stenotic or occlusive lesions in the management of peripheral arterial disease (PAD) requires the use of nephrotoxic iodine contrast. Iodine contrast in patients receiving a PTA can cause post contrast acute kidney injury. ${ }^{1-3}$ Recent update of the ESUR (European Society of Urogenital Radiology) guidelines changed the definition of contrast induced nephropathy (CIN) to post contrast induced kidney injury (PCAKI) as the preferred term for renal function deterioration after contrast medium. ${ }^{4}$ This protocol will refer to CIN as PC-AKI. PC-AKI is defined as a decrease in estimated glomerular filtration rate (eGFR) of $>25 \%$ compared to baseline values or a rise of $>0.5$ $\mathrm{mg} / \mathrm{dl}$ serum creatinine within 72 hours after an iodine contrast mediated procedure (KDIGO guidelines). ${ }^{5,6}$ Krasznai et al. described an $13 \%$ incidence of PC-AKI in patients treated with a PTA, regardless of prior renal function. ${ }^{7}$ The incidence of PC-AKI can be as high as $50 \%$ in high-risk patients and is the cause of $10 \%$ acute in hospital renal failure. ${ }^{1,8,9}$ Moreover, high-risk patients diagnosed with $\mathrm{CKD}$ are known to have an increased risk of developing PC-AKI after administration of iodine contrast. CKD and iodine contrast are both independent risk factors in the development of PC-AKI. ${ }^{1}$ Furthermore, CKD is a global problem, affecting 10-16 percent of the general population. ${ }^{9}$ Prevalence of CKD is increasing worldwide and is estimated to be as high as $45 \%$ in de population aged $>70$ years. ${ }^{9}$ Moreover, incidence and prevalence of PC-AKI are rising $5-8 \%$ annually. ${ }^{1}$

Contrast induced nephropathy is associated with a significant worse outcome due to increased risk of cardio-vascular events, acceleration to end stage renal failure requiring dialysis and extended hospitalization, causing increased morbidity and mortality. ${ }^{7,10-13}$ Moreover, Ramaswami et al. showed a significant higher mortality rate in patients developing PC-AKI after receiving a coronary angiography compared to patients without PC-AKI (resp. $7.1 \%$ vs. $1.1 \%, \mathrm{n}=1826) .^{3}$

Extended hospitalization and additional care due to PC-AKI is costly. Average cost of one year of dialysis in the Netherlands is estimated to be as high as 80.000 euro. The total annual medical costs for patients diagnosed with PC-AKI in the United States are estimated 700 million to 1 billion dollars. ${ }^{9}, 14,15$ Relevant patient-related risk factors developing PCAKI are; chronic kidney disease, diabetes mellitus, heart failure, old age, anemia, and 
decreased function of the left ventricle. ${ }^{7}$ The cause of contrast-induced nephropathy is attributed by multiple mechanisms. Concisely, free radicals are activated in the kidneys due to hyperosmolar stress after contrast is administered, while vasoconstriction induces diminished blood supply to the kidneys, inducing hypoxemia. ${ }^{16,17}$

\section{Prevention of PC-AKI}

Hydration therapy is the cornerstone in the prevention of PC-AKI in high-risk patients. ${ }^{16-18}$ Patients with an eGFR $<45 \mathrm{ml} / \mathrm{min} / 1.73 \mathrm{~m} 2$ or an eGFR $<60 \mathrm{ml} / \mathrm{min} / 1.73 \mathrm{~m} 2$ with one or more comorbidities (Diabetes Mellitus, Heart failure, $\mathrm{PAD}$ ) will receive pre and post hydration. Perprotocolitiscustomaryinourclinictoadminister $0.9 \% \mathrm{NaCli.v.} 3-4 \mathrm{ml} / \mathrm{kg} / \mathrm{h}$ in uncomplicated high-risk patients for 4 hours pre-and 4 hours post intervention. Complicated high-risk patients with heart- or renal failure (exercise-induced dyspnea, edema, eGFR $<30$ $\mathrm{ml} / \mathrm{min} / 1.73 \mathrm{~m} 2$ ) receive 12 hours pre and post hydration with $0.9 \% \mathrm{NaCl}$ i.v. $1 \mathrm{ml} / \mathrm{kg} / \mathrm{h}$.

Increased diuresis and prevention of dehydration is known to protect patients with CKD for possible PC-AKI. ${ }^{11,16-19}$ However, the volume of administered $\mathrm{NaCl}$ solution is often too low to warrant any form of renal protection. These low volumes are usually motivated by fear of overhydration and pulmonary edema. ${ }^{19}$ Forced diuresis using furosemide in combination with intravenous $\mathrm{NaCl} 0.9 \%$ adjusted to diuresis prevents overhydration and provides a mild protection against developing PC-AKI. ${ }^{20}$ On the contrary, some studies show an increased incidence of PC-AKI after use of diuretics in combination with high volume hydration. Mismatched diuretic forced diuresis can cause vasoconstriction due to intravascular volume depletion and thus concentration of contrast in stead of dilution. ${ }^{19-23}$

\section{Intervention}

To achieve high volume diuresis without risking volume depletion or pulmonary edema in high-risk patients requires a delicate balance. Recent publications regarding the Renalguard system $^{\mathrm{TM}}$ show promising results preventing PC-AKI in patients receiving a coronary intervention. ${ }^{18,24-28}$ The Renalguard system ${ }^{\mathrm{TM}}$ is an infusion system regulating volume of $\mathrm{NaCl} 0.9 \%$ administered based on the volume of urine produced. Pre-procedure patients receive a $250 \mathrm{ml} \mathrm{NaCl} 0.9 \%$ bolus in combination with a dose furosemide $(0.5 \mathrm{mg} / \mathrm{kg})$. The goal is to achieve diuresis of $>300 \mathrm{ml} / \mathrm{h}$ before commencing and maintaining output during 
the procedure. Marenzi et al proved Renalguard ${ }^{\mathrm{TM}}$ controlled furosemide forced diuresis with matched hydration to be safe and effective in maintaining adequate intravenous volume. ${ }^{18}$ The MYTHOS-trial demonstrated a reduction PC-AKI in $74 \%$ of patients known with CKD, receiving iodine contrast for diagnostic purposes. ${ }^{18}$ Moreover, Briguori et al. showed an optimal diuresis threshold of $>450 \mathrm{ml} / \mathrm{h}$ with a minimum of $>300 \mathrm{ml} / \mathrm{h}$ to achieve optimal protection against PC-AKI. ${ }^{27}$ Previous studies with the Renalguard ${ }^{\mathrm{TM}}$ did not report any life-threatening events and no serious electrolyte disturbances were mentioned. ${ }^{27,28}$ Briguori et al. described an asymptomatic hypokalemia in 7.5\% (30/400) of patients, in which only $4 \%(16 / 400)$ required potassium supplementation. No significant alterations of sodium levels were observed. ${ }^{27,28}$ Nor was there a significant difference in incidence of pulmonary edema. ${ }^{28}$ However, all previous mentioned research is conducted in a population requiring cardiac diagnostic procedures and therapeutic interventions. No evidence is available using furosemide forced diuresis with matched hydration in combination with the Renalguard infusion system ${ }^{\mathrm{TM}}$ to decrease incidence of PC-AKI in patients with CKD receiving a PTA of the lower extremities.

\section{Diagnosing PC-AKI}

Current diagnosis of PC-AKI relies on rise of serum creatinine 48-72 hours post intervention. However, patients receiving a PTA are often discharged within 24 hours post procedure. Although patients are instructed to return to the clinic for routine control of serum creatinine 3 days post intervention, this is often dismissed. Early detection of acute kidney injury (AKI) or PC-AKI is based on the slow rise in serum creatinine and therefore is an inadequate diagnostic tool. ${ }^{29-31}$ In the past decade several studies tried to identify urine biomarkers for early detection of AKI. ${ }^{31-33}$ Potential biomarkers are neutrophil gelatinaseassociated lipocalin (NGAL), interleukin-18 (IL-18), kidney injury molecule-1 (KIM-1), cystatin $\mathrm{C}$, liver fatty acid binding protein (L-FABP), N-acetyl-beta-D-glucosaminidase (NAG), pi-glutathione-S-transferase ( $\pi$-GST), and tissue inhibitor of metalloproteinase-2 (TIMP-2). ${ }^{31,32}$ One of the more promising urine biomarkers to detect AKI is NGAL. ${ }^{30}$ Rise in NGAL concentration is greatest 4-6 hours post intervention, with an increase up to 25 times compared to baseline value. ${ }^{30}$ 


\section{Study hypothesis}

Our primary hypothesis is that a significant reduction in the incidence of PC-AKI can be established by increasing diuresis $(>300 \mathrm{ml} / \mathrm{h}$ ), using furosemide forced diuresis with matched hydration controlled with the Renalguard system ${ }^{\mathrm{TM}}$ in patients with $\mathrm{CKD}$ receiving an endovascular intervention of the lower extremities. Our second primary hypothesis is that sampling of urine biomarkers (NGAL, KIM-1 en IL-18) 4 hours post intervention can predict PC-AKI in an early stage in patients with CKD compared to rise in serum creatinine 72 hours post intervention.

\section{Methods and Analysis}

\section{Study design}

This study (Protocol V.2.0, date 13 December 2016) is a non-blinded, single centre prospective randomized controlled trial. The patients will be included in the 'Zuyderland' Medical Centre, Heerlen, the Netherlands. Patients with a diminished renal function (eGFR $<60 \mathrm{ml} / \mathrm{min} / 1.73 \mathrm{~m} 2$ ) diagnosed with $\mathrm{PAD}$ and in need of an endovascular intervention of the lower extremities will be included. Patients participating in this study will not require extended hospitalization or additional follow up compared to standard of care. Serum creatinine is obtained within 10 days prior to procedural and post procedure on day 1, 3 and 30 (Figure 1. Flow chart of the study). Obtaining these serum creatinine samples is standard of care. Estimated glomerular filtration rate (eGFR) is calculated using the adjusted formula by Levey et al. ${ }^{34}$ Pre- and post hydration in the control group are administered as dictated by hospital protocol. Patients will receive peripheral venous access for administration of $\mathrm{NaCl} 0.9 \%$. Furthermore, a Foley catheter will be placed to monitor diuresis. Not within standard of care is administering furosemide $(0.5 \mathrm{mg} / \mathrm{kg})$ in the intervention group in conjunction with a bolus $\mathrm{NaCl} 0.9 \%(250 \mathrm{ml})$ to increase diuresis. Use of furosemide is a medicine registered to increase diuresis in treatment of edema associated with renal disease including nephrotoxic syndrome, congestive heart failure, and liver cirrhosis.

To observe reduction in PC-AKI we compare patients treated with furosemide forced diuresis with matched hydration to a control group. Control group will receive standard 
of care pre and post hydration (described in intervention and comparison). This trial is registered with the Netherlands Trial Register.nl (NTR6236), registration date. The total study period is two years, from April 2018 to March 2020.

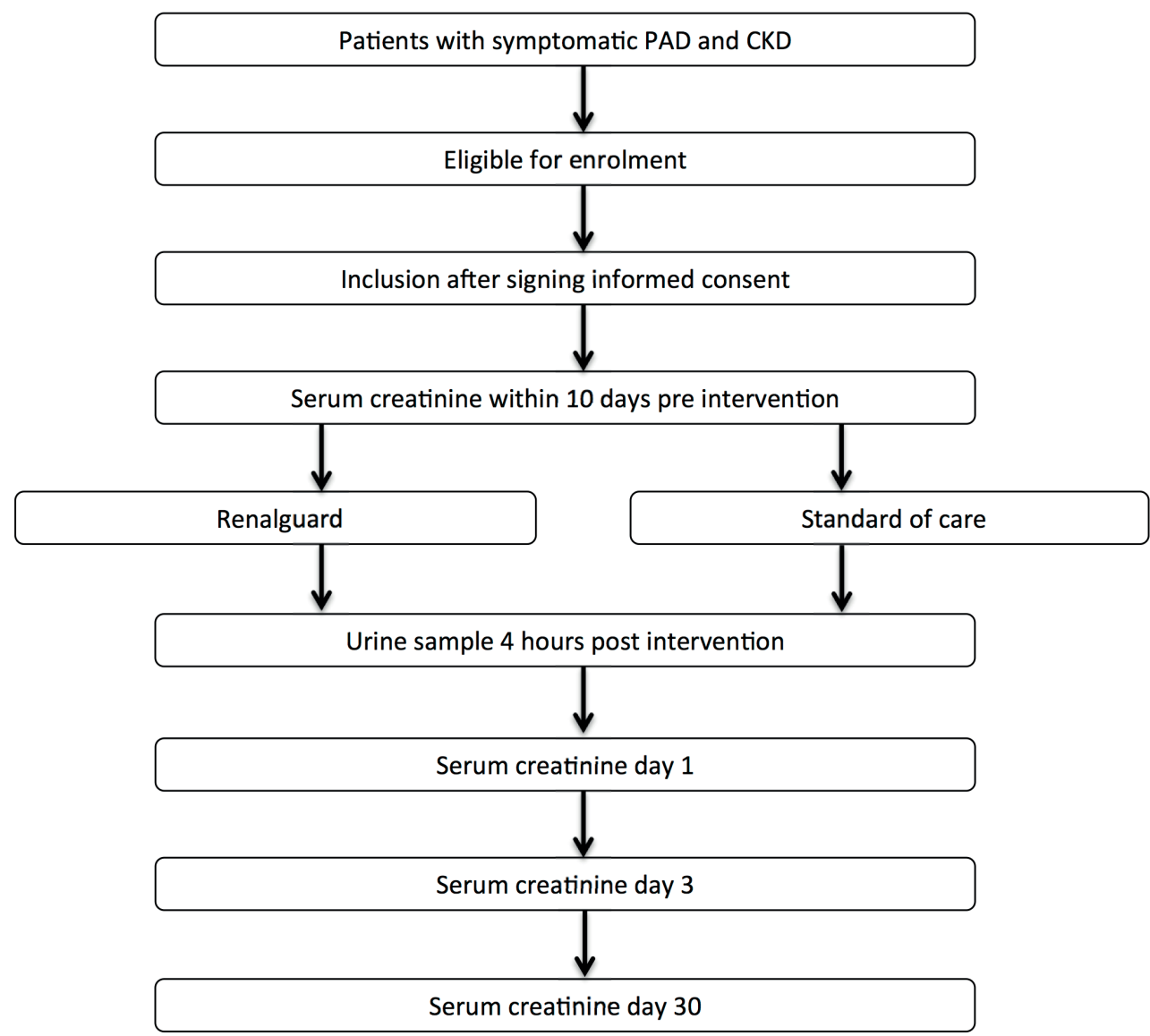

Figure 1. Flow chart of the study. Eligibility based on in- and exclusion criteria. Enrolment by random assignment. Serum creatinine for measurement of renal function pre- and post procedure. Urine sampling to analyse biomarkers post procedure. 


\section{Patient and Public Involvement}

Patient and public were not involved in the design, recruitment to and conduct of the study. The research question was not developed based on patients' priorities, experience or preferences. Results of the study will be disseminated to the study participant upon request.

\section{Outcome measurements}

Primary endpoints are defined as the incidence of PC-AKI, 3 days after a successful endovascular procedure of the lower extremities. Serum creatinine is measured post intervention on day 1, 3 and 30. Patients are required to return to the hospital for blood samples at day 3 and day 30 . PC-AKI is defined as a decrease in eGFR $>25 \%$ or rise in serum creatinine of $>0.5 \mathrm{mg} / \mathrm{dl}$ compared to baseline values. Primary success is defined as a $50 \%$ reduction in the incidence of contrast induced nephropathy in the Renalguard ${ }^{\mathrm{TM}}$ group using furosemide forced diuresis with matched hydration. Second primary endpoint is rise of urine biomarkers, after successful endovascular intervention of the lower extremities. Positive rise in urine biomarkers (NGAL, IL-18 and/or KIM-1) is defined as an AUCROC (area under the curve ROC) $>0.7$ sampled 4 hours after concluding endovascular procedure. Rise in urine biomarkers will be compared to rise in serum creatinine 72 hours post intervention to see if there is a correlation and early detection of PC-AKI.

Secondary endpoints are complications due to PC-AKI-prophylactic therapy (PC-AKI requiring dialysis (previously not requiring dialysis), serious electrolyte disturbances (requiring addition treatment) and/or acute pulmonary edema (radiologic confirmation and requiring diuretic medication)), post-operative in-hospital adverse events (acute myocardial infarct (confirmed on electrocardiogram), death), length of hospitalization, post-operative complication at home requiring additional care (seroma, wound infection, pseudo aneurysm and re-occlusion or re-stenosis within 4 weeks after intervention). Complications will be registered in the days post intervention while hospitalized and evaluated 4 weeks after intervention in the outpatient clinic by a vascular surgeon, unaware to allocated treatment. The follow up data will be collected and processed by a member of the study team, not blinded to allocated treatment. It should be mentioned that this protocol is not powered to detect significant differences in the incidence of adverse events between the two treatment groups. 


\section{Other clinical study parameters}

The following baseline parameters will be collected: age, gender, ethnicity, height, weight, diabetes mellitus (defined as receiving anti diabetic treatment, not diet-controlled), hypertension (defined as a systolic pressure $>140 \mathrm{mmHg}$ (measured at the preoperative work up of the anesthetist) or use of anti hypertensive medication), heart failure (defined as an ejection fraction $<40 \%$ ), baseline renal function (acquired at standard preoperative assessment, $<10$ days of intervention). The following operative data are collected: location of stenosis/occlusion (iliac, femoral, BTK or multi-level), OR-time, radiation dose, radiation time, volume of contrast, volume of $\mathrm{NaCl} 0.9 \%$ administered ( 90 minutes pretill four hours post- intervention). Table 1 . Schedule of enrolment.

Table 1 Schedule of enrolment, intervention and assessment

\begin{tabular}{|c|c|c|c|c|c|c|c|}
\hline \multicolumn{8}{|c|}{ Study Period } \\
\hline Process Time point & $\begin{array}{l}\text { Screening, } \\
\text { enrolment } \\
\text { and } \\
\text { allocation }\end{array}$ & $\begin{array}{l}\text { Pre intervention } \\
\text { (10 days) }\end{array}$ & Intervention & $\begin{array}{l}\text { FU + } \\
\text { 4hour }\end{array}$ & $\begin{array}{l}\text { FU + } \\
\text { 1day }\end{array}$ & $\begin{array}{l}\mathrm{FU}+3 \\
\text { days }\end{array}$ & $\begin{array}{l}\text { FU } \\
+30 \\
\text { days }\end{array}$ \\
\hline
\end{tabular}

\begin{tabular}{|c|c|c|c|c|c|c|c|}
\hline \multicolumn{8}{|l|}{$\begin{array}{l}\text { Screening, enrolment } \\
\text { and allocation }\end{array}$} \\
\hline Eligibility screen & $x$ & & & & & & \\
\hline Informed consent & $x$ & & & & & & \\
\hline Baseline parameters & $x$ & & & & & & \\
\hline Vital signs & $x$ & & $x$ & $x$ & $x$ & $x$ & $\mathrm{x}$ \\
\hline \multicolumn{8}{|l|}{ Intervention } \\
\hline Operative data & & & $x$ & & & & \\
\hline Renalguard $^{\mathrm{TM}}$ & $x$ & $x$ & $x$ & $x$ & $x$ & $x$ & $x$ \\
\hline Standard of care & $\mathrm{x}$ & $x$ & $x$ & $\mathrm{x}$ & $x$ & $x$ & $x$ \\
\hline \multicolumn{8}{|l|}{ Assessment } \\
\hline Urine biomarkers & & & & $x$ & & & \\
\hline Serum creatinine & & $x$ & & & $x$ & $x$ & $x$ \\
\hline \multicolumn{8}{|l|}{ Outcome measurements } \\
\hline Primary & & & & $x$ & $x$ & $x$ & $\mathrm{x}$ \\
\hline Secondary & & & $x$ & $x$ & $x$ & $x$ & $x$ \\
\hline
\end{tabular}


Study population: Patients with chronic kidney disease (eGFR $<60 \mathrm{ml} / \mathrm{min} / 1.73 \mathrm{~m} 2$ ) diagnosed with PAD requiring a PTA of the lower extremities.

Inclusion criteria

- Patients at least 18 years of age

- Diagnosed with occlusive or stenotic peripheral arterial disease requiring an endovascular intervention with contrast.

- $\quad$ eGFR $<60 \mathrm{ml} / \mathrm{min} / 1.73 \mathrm{~m} 2$

Exclusion criteria

- Hypersensitivity to furosemide

- Use of intravenous contrast within 10 days prior to qualifying intervention

- Expected to receive intravenous contrast within 72 hours after qualifying intervention

- Unable to receive a Foley catheter

\section{Sample size calculation}

Sample size is based on a randomized controlled trial comparing standard hydration therapy with Renalguard ${ }^{\mathrm{TM}}$ controlled furosemide forced diuresis with matched hydration in patients with CKD receiving a coronary procedure. ${ }^{18}$ Incidence of PC-AKI in the Renalguard $^{\mathrm{TM}}$ group was $4.6 \%$ compared to $18 \%$ in the control group (standard of care hydration therapy). Based on these results a sample size is calculated with a significance level of $5 \%$ and a power of $80 \%$. Sample size is estimated to include 86 patients in each group, with a total sample size of 172 patients. Taking into account a possible lost to follow-up or early withdrawal of 5\%, a total sample size of 180 patients is required. 


\section{Randomization and concealment}

Randomization will be performed using a randomization program (http:/www. graphpad.com/quickcalcs/randomize2.cfm). Randomization will be performed prior to first inclusion. Patients will be assigned treatment in consecutive order as dictated by the randomization list. Included patients will be allocated a unique study number. When written consent is acquired a second study member will be approached for the randomization, unaware of patient characteristics to minimize selection bias. Allocation to a treatment group and study number will be registered in a password-protected document only accessible for the principle- and coordinating investigator. Blinding of patients and study members is not possible, as patients in the intervention group will be treated with the Renalguard infusion system ${ }^{\mathrm{TM}}$ during and continuing 4 hours post intervention. The Renalguard infusion system ${ }^{\mathrm{TM}}$ is installed prior to intervention. The control group will receive pre-hydration 4 hours prior to intervention and 4 hours post intervention.

\section{Recruitment of participants}

When referred by general practitioner patients will receive an ankle brachial index (ABI) and duplex-ultrasound of the lower extremities prior to first presentation in the outpatient clinic. Up to Rutherford classification III patients will innately be treated with supervised exercise therapy (SET). When not responding to SET an MRA is performed. All patients with peripheral arterial disease (PAD) (non-responders to SET and Rutherford IV-VI) with a new MRA will be discussed in a multi-disciplinary meeting of vascular surgeons and interventional radiologists. Treatment options are discussed and a plan of approach is formulated. If the patient qualifies for an endovascular intervention and is eligible to be included in this study, a member of the study group will provide information regarding the study orally and on paper. A week after the information is provided a member of the research group will call the patient and inquires whether the patient is willing to participate in the study. After oral confirmation the patient is required to provide written consent at the outpatient clinic before randomization (Figure 1. Flow chart of the study). If the patient does not wish to participate in the study he/she will be scheduled for a regular procedure according to standard of care. This decision will not influence quality of treatment nor will there be any resentment towards the patient. 
The Renalguard system ${ }^{\mathrm{TM}}$ is consists of a console and (disposable) RenalGuard ${ }^{\mathrm{TM}}$ set for infusion and urine collection. The disposable set contains a urine collection set that can be connected to a standard Foley catheter and an infusion set that can be connected to a standard IV catheter. The console weights the volume of urine produced in the collection set and administers an equal amount of hydration fluid $(\mathrm{NaCl} 0.9 \%)$ to match diuresis. The console relies on a patented software and electronic weight measurements to adjust velocity in which hydration fluid is administered as well as monitoring of diuresis. The console is mounted on an adjustable IV pole and is equipped with an internal battery enabling the console to keep functioning during transport from ward to operating theatre and vice versa.

\section{Intervention and comparison}

Nephrotoxic medications (NSAID, metformin) are ceased on the day of intervention. Preand post hydration in the control group does not differ from current clinical treatment. On the day of intervention, the patients will report on the pre-operative ward at 7.30. Patients are instructed to stop oral intake after 24.00 the day before intervention. Oral fluids before 24.00 are permitted. Patients are prepped according to local protocol. An IV line and a Foley catheter are placed to administer fluids and monitor diuresis. Uncomplicated high-risk patients receive 4 hours pre- and 4 hours post hydration with $0.9 \% \mathrm{NaCl}$ i.v. 3-4 $\mathrm{ml} / \mathrm{kg} / \mathrm{h}$. Complicated high-risk patients due to heart- or renal failure (exercise-induced dyspnea, edema, eGFR $<30 \mathrm{ml} / \mathrm{min} / 1.73 \mathrm{~m} 2$ ) receive 12 hours pre- and post- hydration with $0.9 \% \mathrm{NaCl}$ i.v. $1 \mathrm{ml} / \mathrm{kg} / \mathrm{h}$. Hydration therapy in the control group is administered as dictated by hospital protocol. Endovascular intervention will be performed in a hybrid operating theatre by one of three vascular surgeons. After concluding the procedure patients will be transported to the general ward. Regular controls will be performed according to hospital protocol. Four hours after procedure the urimeter will be emptied, thereafter the urine produced in 15 minutes is collected for analysis. Once urine is collected the Foley catheter will be removed. Serum creatinine is obtained one-day post intervention. If there are no complications and spontaneous micturition is observed the patient will be discharged. Three days post-intervention the patient is instructed to have a blood sample taken (in the hospital) to establish serum creatinine. Follow-up will be performed by one of the vascular surgeons. Four weeks after intervention patients will have a routine outpatient 
control. Prior to this follow-up moment patients will receive a control duplex to evaluate treated lesion. Furthermore, serum creatinine is measured four weeks post intervention.

Patients in the intervention group will be prepped in a similar fashion. However, after placing an IV line and Foley catheter the Renalguard system ${ }^{\mathrm{TM}}$ will be connected. 90 minutes prior to intervention the patients receive $250 \mathrm{ml} \mathrm{NaCl} 0.9 \%$ IV in 30 minutes. After $\mathrm{NaCl}$ is administered the patient will receive furosemide $(0.5 \mathrm{mg} / \mathrm{kg})$ intra venous. If observed diuresis exceeds $300 \mathrm{ml} / \mathrm{h}$ the patient is ready for procedure. To maintain diuresis of $>300 \mathrm{ml} / \mathrm{h}$ an additional dose furosemide can be administered up to a maximum dose of $2 \mathrm{mg} / \mathrm{kg}$. According to national guidelines the maximum dosage furosemide for adults (IV/oral) should not exceed $1500 \mathrm{mg} /$ day. The total dosage administered in the study is well below maximum. The Renalguard ${ }^{\mathrm{TM}}$ will remain in situ up to 4 hours after the intervention is concluded. After removal of the Renalguard ${ }^{\mathrm{TM}}$ the urimeter will collect the urine production for 15 minutes for analysis. Thereafter postoperative treatment is similar to the control group.

Urine samples collected for analysis will be stored at a temperature of $4^{\circ} \mathrm{C}$ till processing. Urine will be centrifuged for 10 minutes at a speed of $3000 \mathrm{rpm}$. The supernatant will be stored in $500 \mu \mathrm{L}$ aliquots at a temperature of $-80^{\circ} \mathrm{C}$ till further analysis. After completion of the study all urine samples are thawed and analyzed using enzyme-linked immunosorbent assay (ELISA) kits to measure each individual urine biomarker. ${ }^{24,34}$

\section{Data collection and monitoring}

Baselinedataandstudyresultswillbecollected and reported on papercase reportforms (CRFs). The CRFs are created prior to study initiation. The CRFs will be stored in a secure cabinet. The principle investigator (PI) and coordination investigator will be the only researchers with access to these files. Data will be summarized in an SPSS file for further analysis. All included patients will receive an anonymized study number. Coded data will be stored in a password-protected excel-file. This file will only be accessible to the PI and coordinating investigator. Healthcare inspectors, auditors, monitors and members of the medical ethical commission might be granted access to the source data on request as is prescribed by the law. Data and urine samples are treated as dictated by the 'code of conduct' for adequate use and secondary use of human tissue and use of data in healthcare research (Foundation Federation of Dutch Medical Scientific Societies). ${ }^{35}$ Data will be stored for the duration of 15 years after conclusion of the study. 


\section{Statistical analysis}

The results of this study will be collected and analyzed in a secure database. Database will receive a periodical back up. Only members of the research group and licensed authorities will be able to access the database.

Baseline and per operative characteristics are presented as means and standard deviations or median and interquartile ranges as is common for continues variables and as percentages for categorical variables.

Intention-to-treat analysis will be conducted on the final data. The primary outcome is based on the incidence of PC-AKI and will be presented in a contingency table. Statistical tests for significance will be performed using the Chi-square test for categorical variables. Continues variables are compared using the one-way ANOVA or the Kruskal-Wallis test. Furthermore, proportion comparison (z-test) or calculations for odds-ratios will be performed. Risk factors for PC-AKI, increased urine biomarker concentrations and fast renal decline are evaluated using multivariate logistic regression analysis.

Receiver operating characteristic (ROC) curves of the urine biomarkers for early detection of PC-AKI are calculated, as well as 'area under the curve' (AUC ROC) with correlating standard error. Urine biomarkers are evaluated for their diagnostic accuracy for clinical use if lower $99 \%$ confidence interval is $>0.70$. Patients with missing primary outcome data (complete case analysis) will be excluded. Whereas, sensitivity analysis with multiple imputations (mean of 5 imputations) will be performed for missing values other than primary outcome data. Optimal cut-off point for urine biomarker values for diagnosing PC-AKI and corresponding sensitivity and specificity are calculated assuming false positive and false negative result are of equal clinical importance using the following formula: Sensitivity - ((1 - Prevalence) / Prevalence $)^{*}(1-$ Specificity $)$.

Clinical outcome of patients are compared to four categories (no PC-AKI and normal biomarkers, no PC-AKI and increased biomarkers, PC-AKI and normal biomarkers, PCAKI and increased biomarkers). Statistical analysis will be performed by L.J.J.B. using SPSS (IBM Corp, Armonk, New York, V.21.0). 


\section{Adverse Events}

All adverse events (AE's) observed by the study subject or by a member of the research group are noted and filed. Serious adverse events (SAE's) are unexpected medical events or effect with potential risk of; death, life threatening, hospitalization or extended hospitalization, chronic impairment, or other important medical occurrences potentially harming the patient or requiring an intervention to advert one of the previously mentioned outcomes. SAE's occurring within 4 weeks after intervention are required to be reported to the research ethics committee (REC). The primary endpoint in this study is defined as PCAKI 3 and 30 days post-intervention and accounts for the limited period in which SAE's need to be reported. SAE's that occur within the 30 days post intervention are reported within 15 days. If a patient dies or a life-threatening situation unfolds, the REC needs to be notified within 7 days. If health of included patients is at risk, the study will be stopped and REC will be notified. In this period the REC will investigate possible risks. (S)AE's will be followed until a stable situation is created or the SAE is resolved.

\section{Ethics and dissemination}

The study protocol was submitted and approved by the research ethics committee (REC) and the institutional research board (Zuyderland Medical Centre, Heerlen). This trial will be conducted following the Good Clinical Practice Guidelines, the declaration of Helsinki ( $7^{\text {th }}$ amendment, October 2013) and in accordance with national legislation (Medical Research Act). Substantial amendments to the study protocol will be re-submitted to the original research ethics committee. It is not required to submit a non-substantial amendment to the REC, however a note to file is created and archived by the investigator. A substantial amendment is defined as an alteration to the originally submitted study protocol or supporting document with high probability to impact: safety or the physical or psychological integrity of the study subject, scientific value of the study, conducting or management of the study, quality or safety of one of the interventions in the study. All substantial amendments are submitted to the REC of initial approval of the study protocol. Research findings will be submitted for publication in a PubMed-indexed medical journal within one year after inclusion of the last patient. If the study manuscript is not accepted for publication the research findings will be made publically available on the Internet. 


\section{Discussion}

Total period of inclusion will be two years and is expected to finish May 2020. Study results will clarify whether furosemide forced diuresis with matched hydration using the Renalguard system ${ }^{\mathrm{TM}}$ is superior in the prevention of PC-AKI compared to standard of care hydration therapy in patients with CKD. Furthermore, this study will define whether urine biomarkers, NGAL, IL-18 and KIM-1, are adequate biomarkers in detection of PC-AKI within 4 hours post intervention compared to serum creatinine after 72 hours.

Outcomes reported from a systematic review and meta-analysis of randomized controlled trials show furosemide forced diuresis with matched hydration using the Renalguard system $^{\mathrm{TM}}$ in patients undergoing interventional procedures to significantly decrease the need for renal replacement therapy. ${ }^{28}$ However, all included trials performed coronary interventions or percutaneous aortic valve replacement. No literature is available using furosemide forced diuresis with matched hydration in patients treated endovascular for symptomatic PAD. Nor is any previous research available using the Renalguard system $^{\mathrm{TM}}$ in patients with PAD. Safety evaluation of the Renalguard system ${ }^{\mathrm{TM}}$ in the previous mentioned systematic review showed no increased risk of electrolyte imbalance or pulmonary edema compared to conservative treatment. ${ }^{28}$ However, the meta-analysis included only four trials with high risk for bias. Larger RCT's are needed to exemplify possible effectiveness in endovascular interventions other than coronary procedures.

PC-AKI is diagnosed by a gradual increase of serum creatinine concentration within the first days after endovascular procedure. ${ }^{5,6}$ Delay in diagnosis due to slow increase in serum creatinine makes it an inadequate marker in the early detection of PC-AKI. As previously mentioned in this protocol, patients are often discharged before serum creatinine can be assessed 48-72 hours post-intervention. Despite instructions to return for serum creatinine controls, patient often refrain from follow-up. Evaluating urine biomarkers 4 hours post intervention might possibly address this matter and enable us to detect PC-AKI in an early stage. Use of urine biomarkers depends on the diagnostic accuracy of the studied urine biomarkers and whether they are sufficiently high. Although PC-AKI rarely requires renal replacement therapy, early detection of PC-AKI increases awareness and provides an opportunity to closely monitor renal function and intervene immediately if necessary without delay. 
In this RCT, we will include patients with CKD who qualify for an endovascular intervention of the lower extremities, regardless of anatomic location. Patients can be treated solely with angioplasty or with additional stenting. Consideration for additional stenting will transpire perioperative. The decision to include only patients with CKD was made based on previous literature proving renal replacement therapy is rarely needed in patients diagnosed with PC-AKI but without $\mathrm{CKD} .{ }^{36} \mathrm{PC}$-AKI requiring renal replacement therapy is prevalent in $1 \%$ of the patients without $\mathrm{CKD}$, compared to $7 \%$ in patients with CKD. ${ }^{37}$

This trial is the first to investigate whether furosemide forced diuresis with matched hydration using the Renalguard system ${ }^{\mathrm{TM}}$ can reduce the incidence of PC-AKI in patients with CKD and PAD receiving a PTA of the lower extremities. Furthermore, this study is the first study to establish the use of urine biomarkers in patients receiving a PTA in the detection of PC-AKI compared to serum creatinine.

It is anticipated that study results will provide a solution for early detection of contrast induced nephropathy and offer a preventive measure in patients with chronic kidney disease receiving a PTA of the lower extremities. Study results will be disseminated by oral presentation at conferences and will be submitted to a peer-reviewed journal.

\section{Funding statement}

This research received no specific grant from any funding agency in the public, commercial, or not-for-profit sectors.

Trial registration number: NTR6236

EudraCT number: 2016-005072-10 


\section{References}

1. Parfrey PS, Griffiths SM, Barrett BJ, Paul MD, Genge M, Withers J, et al. Contrast materialinduced renal failure in patients with diabetes mellitus, renal insufficiency, or both. A prospective controlled study. N Engl J Med. 1989 Jan 19;320(3):143-9.

2. Cluley SR, Brener BJ, Hollier L, Schoenfeld R, Novick A, Vilkomerson D, et al. Transcutaneous ultrasonography can be used to guide and monitor balloon angioplasty. J Vasc Surg. 1993 Jan;17(1):23-30-31.

3. Ramaswami G, Al-Kutoubi A, Nicolaides AN, Dhanjil S, Vilkomerson D, Ferrara-Ryan M, et al. Angioplasty of lower limb arterial stenoses under ultrasound guidance: single-center experience. J Endovasc Surg Off J Int Soc Endovasc Surg. 1999 Feb;6(1):52-8.

4. van der Molen AJ, Reimer P, Dekkers IA, Bongartz G, Bellin M-F, Bertolotto M, et al. Postcontrast acute kidney injury - Part 1: Definition, clinical features, incidence, role of contrast medium and risk factors: Recommendations for updated ESUR Contrast Medium Safety Committee guidelines. Eur Radiol. 2018 Jul;28(7):2845-55.

5. Khwaja A. KDIGO clinical practice guidelines for acute kidney injury. Nephron Clin Pract. 2012;120(4):c179-184.

6. Palevsky PM, Curhan GC, Sheridan AM. Definition of acute kidney injury (acute renal failure). Uptodate.com; 2014.

7. Sigterman TA, Krasznai AG, Snoeijs MG, Heijboer R, Schurink GWH, Bouwman LH. Contrast Induced Nephropathy and Long-term Renal Decline After Percutaneous Transluminal Angioplasty for Symptomatic Peripheral Arterial Disease. Eur J Vasc Endovasc Surg Off J Eur Soc Vasc Surg. 2015 Oct 9;

8. McCullough PA, Wolyn R, Rocher LL, Levin RN, O’Neill WW. Acute renal failure after coronary intervention: incidence, risk factors, and relationship to mortality. Am J Med. 1997 Nov;103(5):368-75.

9. Coresh J, Selvin E, Stevens LA, Manzi J, Kusek JW, Eggers P, et al. Prevalence of chronic kidney disease in the United States. JAMA. 2007 Nov 7;298(17):2038-47.

10. Levy EM, Viscoli CM, Horwitz RI. The effect of acute renal failure on mortality. A cohort analysis. JAMA. 1996 May 15;275(19):1489-94.

11. Hoste EAJ, De Corte W. Clinical consequences of acute kidney injury. Contrib Nephrol. 2011;174:56-64.

12. Rihal CS, Textor SC, Grill DE, Berger PB, Ting HH, Best PJ, et al. Incidence and prog nostic importance of acute renal failure after percutaneous coronary intervention. Circulation. 2002 May 14;105(19):2259-64.

13. Best PJM, Lennon R, Ting HH, Bell MR, Rihal CS, Holmes DR, et al. The impact of renal insufficiency on clinical outcomes in patients undergoing percutaneous coronary interventions. J Am Coll Cardiol. 2002 Apr 3;39(7):1113-9.

14. Sadat U, Usman A, Boyle JR, Hayes PD, Solomon RJ. Contrast Medium-Induced Acute 
Kidney Injury. Cardiorenal Med. 2015 Jun;5(3):219-28.

15. Calculating the cost. Interview with Marion Kerr. Health Serv J. 2011; Suppl 1:3.

16. Trivedi HS, Moore H, Nasr S, Aggarwal K, Agrawal A, Goel P, et al. A randomized prospective trial to assess the role of saline hydration on the development of contrast nephrotoxicity. Nephron Clin Pract. 2003 Jan;93(1):C29-34.

17. Mueller C, Buerkle G, Buettner HJ, Petersen J, Perruchoud AP, Eriksson U, et al. Prevention of contrast media-associated nephropathy: randomized comparison of 2 hydration regimens in 1620 patients undergoing coronary angioplasty. Arch Intern Med. 2002 Feb 11;162(3):329-36.

18. Marenzi G, Ferrari C, Marana I, Assanelli E, De Metrio M, Teruzzi G, et al. Prevention of Contrast Nephropathy by Furosemide With Matched Hydration. JACC Cardiovasc Interv. 2012 Jan; $5(1): 90-7$.

19. Pannu N, Wiebe N, Tonelli M, Alberta Kidney Disease Network for the. Prophylaxis Strategies for Contrast-Induced Nephropathy. JAMA. 2006 Jun 21;295(23):2765.

20. Stevens MA, McCullough PA, Tobin KJ, Speck JP, Westveer DC, Guido-Allen DA, et al. A prospective randomized trial of prevention measures in patients at high risk for contrast nephropathy: results of the P.R.I.N.C.E. Study. Prevention of Radiocontrast Induced Nephropathy Clinical Evaluation. J Am Coll Cardiol. 1999 Feb;33(2):403-11.

21. Solomon R, Werner C, Mann D, D'Elia J, Silva P. Effects of saline, mannitol, and furosemide to prevent acute decreases in renal function induced by radiocontrast agents. $N$ Engl J Med. 1994 Nov 24;331(21):1416-20.

22. Weinstein JM, Heyman S, Brezis M. Potential deleterious effect of furosemide in radiocontrast nephropathy. Nephron. 1992;62(4):413-5.

23. Weisberg LS, Kurnik PB, Kurnik BR. Risk of radiocontrast nephropathy in patients with and without diabetes mellitus. Kidney Int. 1994 Jan;45(1):259-65.

24. Dorval J-F, Dixon SR, Zelman RB, Davidson CJ, Rudko R, Resnic FS. Feasibility study of the RenalGuard ${ }^{\mathrm{TM}}$ balanced hydration system: A novel strategy for the prevention of contrastinduced nephropathy in high risk patients. Int J Cardiol. 2013 Jun;166(2):482-6.

25. Chorin E, Ben-Assa E, Konigstein M, Rofe M-T, Hochstadt A, Galli N, et al. Prevention of post procedural acute kidney injury in the catheterization laboratory in a real-world population. Int J Cardiol. 2017 Jan 1;226:42-7.

26. Visconti G, Focaccio A, Donahue M, Golia B, Marzano A, Donnarumma E, et al. RenalGuard System for the prevention of acute kidney injury in patients undergoing transcatheter aortic valve implantation. EuroIntervention J Eur Collab Work Group Interv Cardiol Eur Soc Cardiol. 2016 Apr 8;11(14):e1658-1661.

27. Briguori C, Visconti G, Donahue M, De Micco F, Focaccio A, Golia B, et al. RenalGuard system in high-risk patients for contrast-induced acute kidney injury. Am Heart J. 2016 Mar; 173:67-76.

28. Putzu A, Boscolo Berto M, Belletti A, Pasotti E, Cassina T, Moccetti T, et al. Prevention of Contrast-Induced Acute Kidney Injury by Furosemide With Matched Hydration in Patients Undergoing Interventional Procedures: A Systematic Review and Meta-Analysis of 
Randomized Trials. JACC Cardiovasc Interv. 2017 Feb 27;10(4):355-63.

29. Erdbruegger U, Palevsky PM, Sheridan AM. Etiology and diagnosis of prerenal disease and acute tubular necrosis in acute kidney injury (acute renal failure). Uptodate.com; 2014.

30. Bennett M, Dent CL, Ma Q, Dastrala S, Grenier F, Workman R, et al. Urine NGAL predicts severity of acute kidney injury after cardiac surgery: a prospective study. Clin J Am Soc Nephrol CJASN. 2008 May;3(3):665-73.

31. Erdbruegger U, Palevsky PM, Sheridan AM. Investigational biomarkers and the evaluation of acute tubular necrosis. Uptodate.com; 2014.

32. Cruz DN, Bagshaw SM, Maisel A, Lewington A, Thadhani R, Chakravarthi R, et al. Use of biomarkers to assess prognosis and guide management of patients with acute kidney injury. Contrib Nephrol. 2013;182:45-64.

33. Ronco C, Legrand M, Goldstein SL, Hur M, Tran N, Howell EC, et al. Neutrophil gelatinaseassociated lipocalin: ready for routine clinical use? An international perspective. Blood Purif. 2014;37(4):271-85.

34. Levey AS. A New Equation to Estimate Glomerular Filtration Rate. Ann Intern Med. 2009 May 5;150(9):604.

35. Federatie van Medisch Wetenschappelijke Verenigingen. Verantwoord omgaan met lichaamsmateriaal ten behoeve van wetenschappelijk onderzoek: gedragscode 2011. Rotterdam: FEDERA; 2011.

36. Freeman RV, O'Donnell M, Share D, Meengs WL, Kline-Rogers E, Clark VL, et al. Nephropathy requiring dialysis after percutaneous coronary intervention and the critical role of an adjusted contrast dose. Am J Cardiol. 2002 Nov 15;90(10):1068-73.

37. Gruberg L, Mintz GS, Mehran R, Gangas G, Lansky AJ, Kent KM, et al. The prognostic implications of further renal function deterioration within $48 \mathrm{~h}$ of interventional coronary procedures in patients with pre-existent chronic renal insufficiency. J Am Coll Cardiol. 2000 Nov 1;36(5):1542-8. 



\section{PART III Implications in a non-invasive}

treatment strategy 


\section{СНAPTER 8 Assessment of behavioural determinants influencing success of supervised exercise therapy in patients with}

intermittent claudication: A cross sectional survey

LJJ. Bolt ${ }^{1}$, MLYE. Jacobs ${ }^{1}$, TA. Sigterman ${ }^{2}$, AG. Krasznai ${ }^{1}$, CJJM. Sikkink ${ }^{1}$, GWH. Schurink ${ }^{3}$, LH. Bouwman ${ }^{1}$ 1. Zuyderland Medical Center Parkstad Heerlen, the Netherlands 2. Viecuri Medical Center, Venlo, the Netherlands 3. Maastricht University Medical Center, Maastricht, the Netherlands

Published in:

Physiol Behav. 2020 Mar 1;215:112732 


\section{Abstract}

Background. Supervised exercise therapy is the first step in treatment of intermittent claudication. However, adherence to supervised exercise therapy is low. Limited access and reimbursement issues are known reasons, though lack of motivation is often leading. Behavioral determinants influencing motivation and thus adherence to supervised exercise therapy remain to be investigated. In this study we sought to determine which behavioral determinants would be of influence on the long-term adherence of supervised exercise therapy.

Methods. 200 patients, newly diagnosed with peripheral arterial disease Rutherford classification II-III, were sent a questionnaire to assess motivation and behavior with regard to supervised exercise therapy. The questionnaire was constructed using the I-CHANGE model for explaining motivational and behavioral change. Baseline characteristics were acquired from medical records. Alpha Cronbach's was calculated to test reliability of the questionnaire.

Results. 108 (54\%) patients returned their questionnaire. A total of 79\% patients followed supervised exercise therapy. Patients who increased their walking distance after supervised exercise therapy have significantly greater knowledge $(\mathrm{p}=0.05)$, positive attitude $(\mathrm{p}=0.03)$ and lower negative attitude $(\mathrm{p}=0.01)$. Patients with a higher self-efficacy remained significantly more active after participating in supervised exercise therapy $(\mathrm{p}=0.05)$.

Conclusion. Increasing the determinants knowledge, attitude and self-efficacy will improve adherence to supervised exercise therapy and result in delayed claudication onset time.

\section{Keywords:}

Peripheral arterial disease $(\mathrm{MeSH})$, Exercise therapy $(\mathrm{MeSH})$, Motivation $(\mathrm{MeSH})$, Behavior (MeSH), I-Change Model 


\section{Introduction}

Intermittent claudication (IC) due to peripheral arterial disease often lead to an inactive lifestyle due to pain in the lower extremities and is associated with increased mortality. ${ }^{1-3}$ Conservative treatment using supervised exercise therapy (SET) is recommended as the first step in treatment of intermittent claudication by international guidelines. ${ }^{4}$ SET improves functional performance considerably, prolonging claudication onset time (COT) and peak walking time (PWT) within six months. ${ }^{4-7}$ Several studies have shown that improvement in functional performance after SET is comparable or even superior to endovascular revascularization regarding COT and PWT. ${ }^{7,8}$ Improvement in quality of life is even comparable between iliac stenting and SET after six months. ${ }^{7}$ However, due to low accessibility, reimbursement issues and low patient compliance, SET is often disregarded. ${ }^{6,9}$ Bartelink et al. demonstrated that only $68 \%$ of patients actually participated in supervised exercise therapy after prescription. ${ }^{10}$ This might be due to several disadvantages, such as pain experienced during exercise before actual progress in COT and PWT. Moreover, regular exercise is time consuming and there is no immediate relieve of complaints, in contrast to endovascular interventions. ${ }^{9,10}$ Comorbidities limiting mobility or non-limiting IC symptoms (Rutherford I) are also reasons for low physical exercise compliance. ${ }^{10}$

In this study we sought to determine which behavioral determinants influence longterm adherence to SET and successful prolongation of peak walking distance (PWD) after supervised exercise therapy. Adherence can be described as the degree of a patient's behavior corresponding to the agreed recommended therapy (WHO 2003). Patient participation is key in deciding treatment options. The results of an intervention can be reduced by an inadequate adherence. Several studies involving patients with coronary heart disease showed that motivation and self-efficacy are important determinants in the process to change health behavior and long term adherence to physical exercise. ${ }^{11,12}$ Teixeira et al. identified autonomous motivation, self-efficacy and self-regulating skills as significant mediators for physical activity in patients with obesity. ${ }^{13}$ To assert the cause of low adherence to exercise therapy we sought to determine whether the determinants knowledge, risk perception, attitude, self-efficacy and intention have a significant influence on compliance and outcome measurement PWD. A survey was constructed using the I-CHANGE model to assess these determinants.

The authors hypothesize that patients with greater overall scores in this retrospective survey will improve PWD to a greater extend compared to patients with low scores. It is expected 
that optimizing behavior towards SET will improve adherence and improved adherence will eventually result in greater increase of PWD.

\section{Material and Methods}

With the use of a cross-sectional survey combined with retrospective research of prospective acquired patient characteristics, 200 patients were approached to fill out a questionnaire. Using the questionnaire, we obtained data at one specific point of time, analyzing the adherence at least six months after SET was prescribed. Patients were identified from electronic medical records diagnosed with symptomatic peripheral arterial disease, Rutherford classification II-III [ICD-10 I73.9]. All patients were newly diagnosed with PAD (Rutherford II-III) in the outpatient clinic between January 2014 and June 2015, patients who were prescribed SET in the last six months before sending questionnaires were not included. Rutherford classification II is defined as moderate intermittent claudication with a maximal PWD of 500 meters, whereas class III is defined as severe intermittent claudication with a maximal PWD of 200 meters. The following baseline characteristics were acquired from medical records; age, gender, body-mass-index (BMI), level of education and marital status. A questionnaire was developed using the theoretical framework of the I-change model (ICM). This model is used to explain the abilities needed to perform a certain behavior, based on intention, ability and skills required. The questionnaire was composed out of determinants based on whether they were changeable and for their importance. Although static facts like socio-economic status and distance to SET location are known factors in low adherence to SET, these unchangeable facts are not included in our survey. The survey contained 10 questions regarding; knowledge (19 a/b, $20)$, risk perception $(21 \mathrm{a} / \mathrm{b} / \mathrm{c})$, attitude (22/23), self-efficacy (24) and intention towards physical exercise (25). One question can be itemized in to a maximum of nine items. In other words, the same question asked under different circumstances. A total of 49 items are scored regarding these five behavioral determinants. Twenty additional questions were asked to establish baseline characteristics.

Knowledge was measured on 17 different items $(\alpha=0.74)$ (for example 'the pain in my legs due to vascular stenosis does only reflect the vascular state in my legs, not other parts of my body') and was answered with true or false. Risk perception on the subscale 'susceptible for' with 13 items $(\alpha=0.91)$ ('If I would stop smoking, I would...') was answered on a 5-point Likert-scale. A Likert-scale enables a patient to respond to a statement on a 
symmetric agree-disagree scale to reflect their level of agreement. The attitude of a person can consist out of pro's and con's with regard to the behavior asked, in other words a positive or negative attitude. Positive attitude was measured on 6 items $(\alpha=0.86)$ ('If I walk regularly it will prevent progression of my vascular disease'), negative attitude was measured on 6 items ( $\alpha=0.76$ ) ('Walking regularly will consume a lot of time'), both were scored on a 5-point Likert scale. Self-efficacy was measured with 6 items $(\alpha=0.88)$ (Do you think you will exercise if you experience pain while walking?) and was answered on a 5-point Likert scale. Intention was measured with a single question (I am determined to listen to the doctors advise and will exercise on a daily bases) on a 5-point Likert scale from 1 (definitely not) to 5 (most definitely). Finally, exercise behavior was subjectively measured by the patient with regard to walking distance before and after SET in the outpatient clinic, three months after SET, as is common in our practice. This study was performed with approval of the institute medical ethical committee (METC Z) and carried out in accordance with the declaration of Helsinki. Accompanying the questionnaire was an informed consent form, which was returned together with the questionnaire. 


\section{I-CHANGE Model}

The questionnaire was constructed using the Integrated Model for explaining motivational and behavioral change (I-CHANGE model) developed by de Vries et al. (fig.1) $)^{14}$

Figure 1. I-Change Model

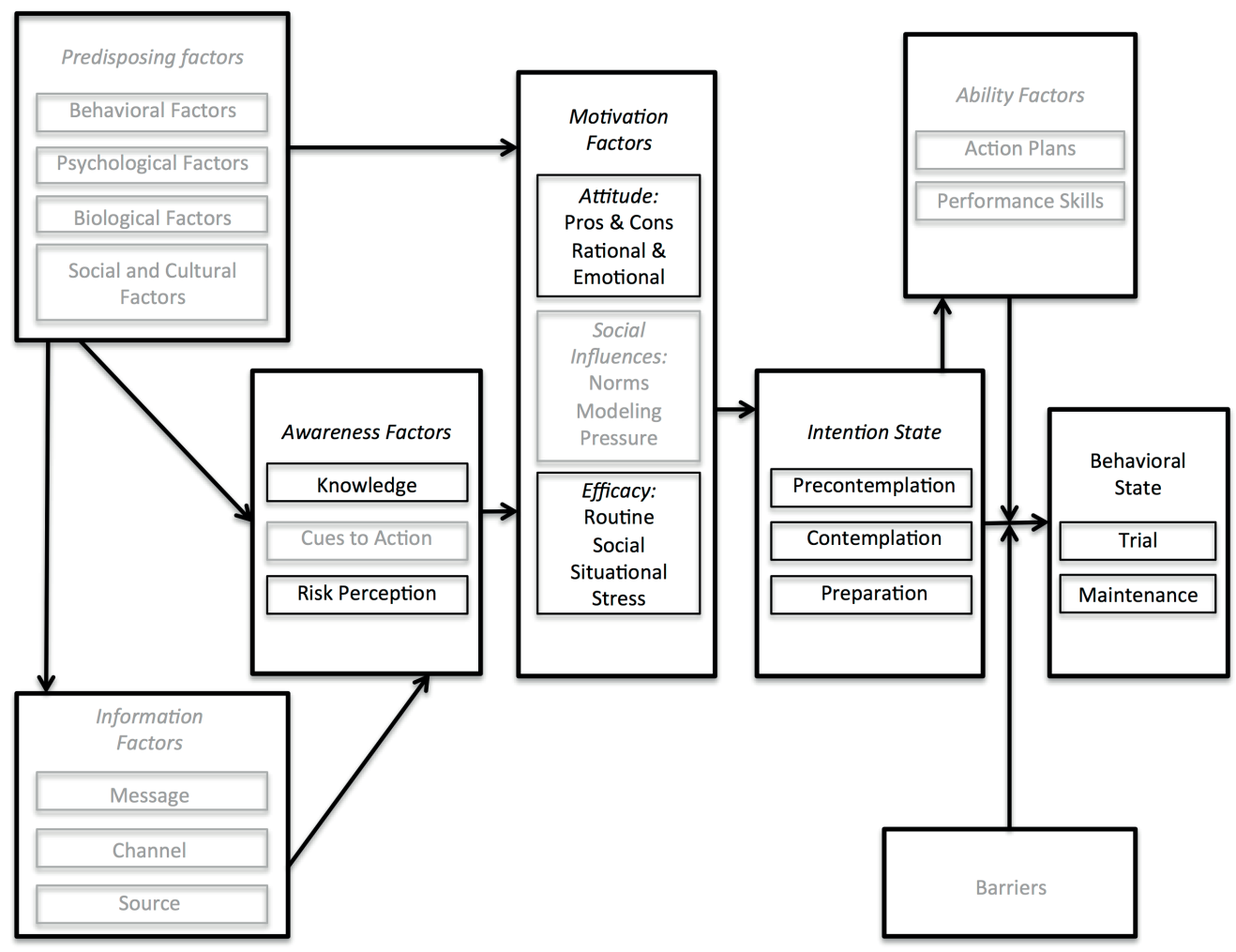

This model states that extroverted and introverted behavior is expressed by a person's intention or motivation to exhibit a certain type of behavior. ${ }^{14,15}$ A person's abilities and intentions determine the expressed behavior. Intentions however can sway rapidly and vary from absent awareness of behavior to considering altering one's behavior. Any intention to alter behavior often exists out of a necessity, for instance complaints of pain interfering in daily activities and quality of life. To display or alter certain behavior a person needs to implement their intentions and requires the ability to prepare and execute deliberate plans, as well as being able to execute the behavioral skills needed. ${ }^{14}$ 
According to the I-change model a person's motivation is determined by three factors: attitude, social influences and self-efficacy. ${ }^{14}$ Attitude is determined by a person's perception of emotional and cognitive benefit or detriment of the behavior. The role of social influences is how a particular type of behavior is perceived by others, values associated with this type of behavior and the covert and or overt feedback from others when exerting this behavior. Self-efficacy is a person's assessment of his own capability to perform a type of behavior. ${ }^{16,17}$ Attitude, social influences and self-efficacy are on their own determined by various distal factors such as awareness (e.g. knowledge, level of intelligence) and predisposing factors: habits, socioeconomic status, psychological factors (cognitive characteristics), social and cultural factors (norms and values) and biological factors (genetic aptitude). ${ }^{15,18}$ Behavior change can be opposed by these various distal factors.

\section{Supervised exercise therapy}

All patients newly presented in our outpatient clinic with PAD, Rutherford classification II-III [ICD-10 I73.9], are forwarded to the physiotherapist to participate in 12 weeks of SET. During the first four weeks patients exercise three times a week. The following eight weeks consists out of two training sessions per week. ${ }^{19}$ After completing the 12 weeks, patients return to the outpatient clinic to evaluate the progression in PWT (peak walking time) and COT (claudication onset time). The patients exercise individually on a treadmill under supervision of a physiotherapist, at the physiotherapists' clinic. The patients are instructed to participate in SET three times a week, one session is 30 minutes. The sessions are tailored. A patient walks at a speed op 3,5 kilometer per hour for as long as he can until the pain becomes unbearable. After the patient stops and de pain has completely resided, the patient will start walking again. This cycle continues until 30 minutes have passed. Exercise progression is measured in the gain in PWT and COT on the treadmill, comparing the first session with the last session after 12 weeks of SET. Beside supervised exercise therapy, patients are stimulated to exercise daily on their own (unsupervised) and apply the same principle as with SET by pushing the PWT as long is possible until stop is required. No additional motivation strategies are applied. The only education patients receive is in the 10 minutes outpatient contact with the physician and an information folder. 


\section{Sample size}

Sample size was calculated using a method published by Andy Field. ${ }^{20}$ To calculate sample size the following formula is used; $50+8 \mathrm{k}$, where $\mathrm{k}$ stands for the number of independent variables, with a desired coincidence interval of $0.05 .{ }^{20}$ The number of independent variables in this questionnaire is 6 , yielding the following result: $50+\left(8^{*} 6\right)=98$ questionnaires. Based on literature response rate is estimated to be $50 \% .{ }^{10}$ Total sample size of patients required to receive a questionnaire was calculated to be 196. A total number of 200 questionnaires were send.

\section{Statistical analyses}

Continuous variables were expressed as means with range and categorical variables expressed as percentages. Missing data were replaced with the mean of five imputations. Baseline characteristics were compared using unpaired t-test for continues variables and chi-square for categorical variables. Logistic regression was applied to identify predictors, predicting whether SET does or does not increase walking distance. Cronbach's Alpha was calculated for each of the six variables, a value of 0.7 was deemed reliable. Cronbach's Alpha was greater than 0.7 for all determinants and thus valid as measurement tool regarding al separate determinants. Results with a P-value less than 0.05 were considered statistically significant. Statistical analysis was performed by L.J.J.B. using SPSS version 21 (SPSS Inc., Chicago, IL).

\section{Results}

In total 200 questionnaires were sent to selected patients, 108 patients (54\%) completed and returned their questionnaires. Mean age was 72 (53-92) years and 62 patients were male (57\%). Patient demographics are presented in table 1. 
Table 1. Demographic characteristics

\begin{tabular}{|c|c|}
\hline Mean age (years) & 72 (range $53-92$ ) \\
\hline Gender (male, \%) & 57 \\
\hline Mean Body mass index & 27 (range $18-61$ ) \\
\hline Smoker (\%) & 41 \\
\hline Diabetes mellitus (\%) & $22 \%$ \\
\hline Hypertension (\%) & $61 \%$ \\
\hline Impaired renal function (MDRD $\left.<60 \mathrm{ml} / \mathrm{min} / 1,73 \mathrm{~m}^{2}\right)(\%)$ & $39 \%$ \\
\hline Lipid-lowering medications (\%) & $54 \%$ \\
\hline Oral anticoagulants & $61 \%$ \\
\hline Acetylsalicylic acid & $77 \%$ \\
\hline Vitamin $\mathrm{K}$ antagonist & $15 \%$ \\
\hline Other anti platelet drug & $6 \%$ \\
\hline Factor $\mathrm{Xa}$ inhibitor & $2 \%$ \\
\hline \multicolumn{2}{|l|}{ Level of education } \\
\hline No education & $2 \%$ \\
\hline Primary school & $20 \%$ \\
\hline Secondary school & $43 \%$ \\
\hline Secondary vocational education & $16 \%$ \\
\hline Higher professional education & $15 \%$ \\
\hline University & $4 \%$ \\
\hline \multicolumn{2}{|l|}{ Marital status } \\
\hline Bachelor & $7 \%$ \\
\hline Married & $65 \%$ \\
\hline Widow/Widower & $26 \%$ \\
\hline Re-married & $2 \%$ \\
\hline
\end{tabular}

Some 79\% ( $\mathrm{n}=85)$ actually did follow supervised exercise therapy, of which 58 (68\%) stated to have increased their PWD (fig.2). Mean duration of SET was 31 (2-52) weeks. Of the 108 patients who returned their questionnaires, 80 (74\%) patients still complained of pain in the lower extremities during exercise, with or without improvement (fig.2). 


\section{Study population $(n=108)$ referred to SET}

- SET with increase PWD $(n=58)$

n SET without increase PWD $(n=27)$

- Did not participate in SET $(n=23)$

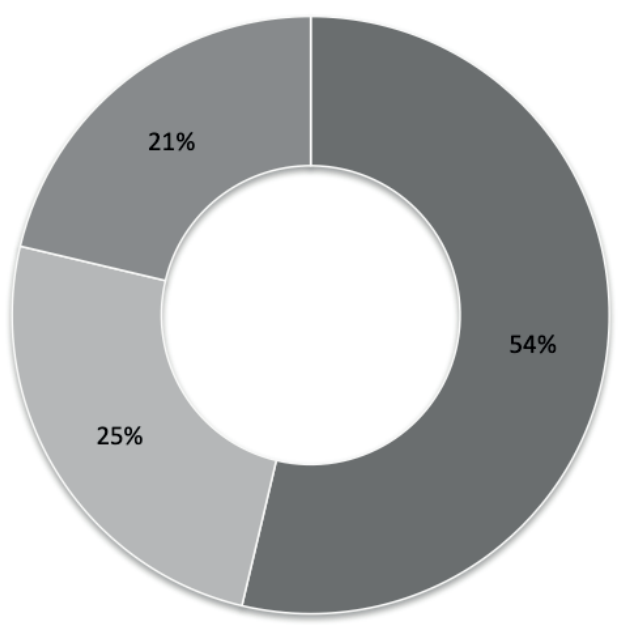

Figure 2. Study population $(n=108)$ referred to SET

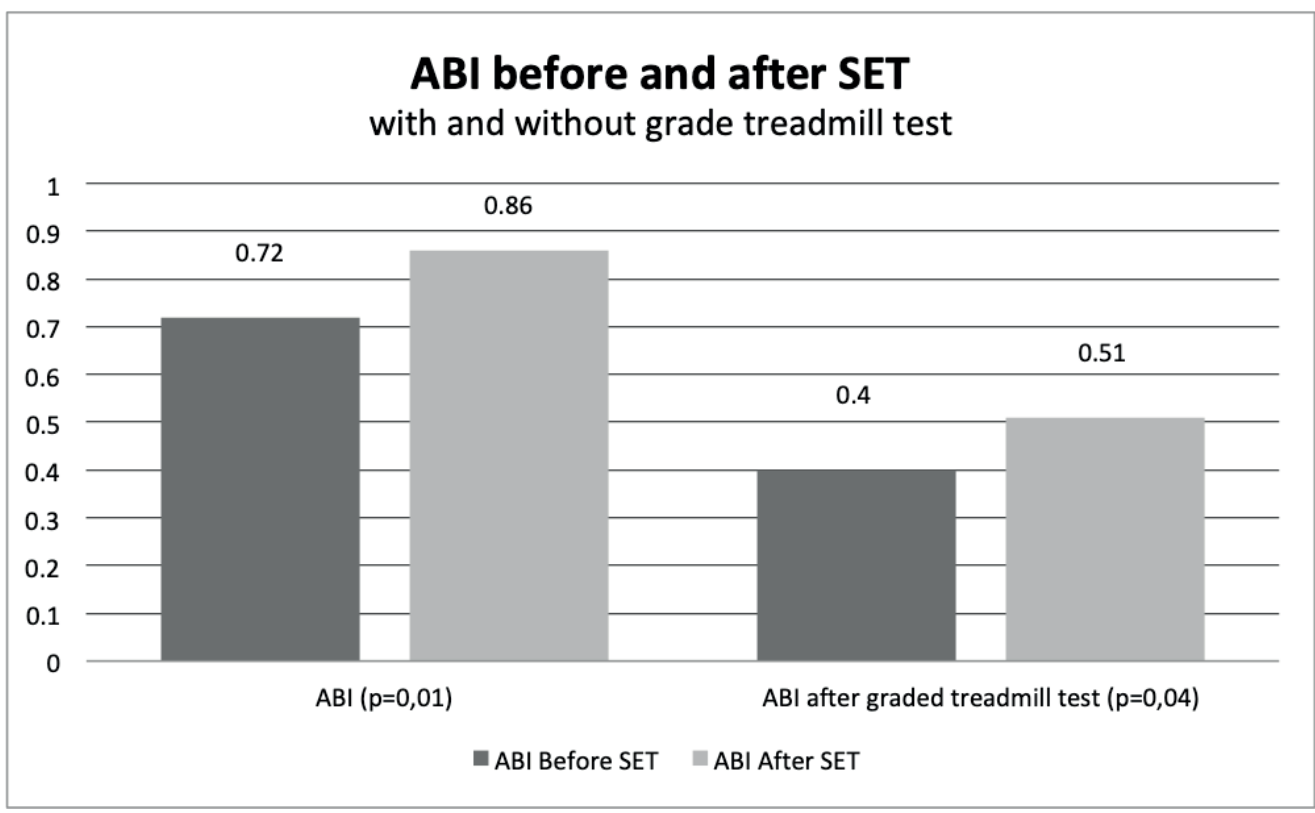

Figure 3. $A B I$ before and after SET 
Mean Ankle-brachial-index at first contact was 0.72 (0.40-1.16) and 0.40 (0.13-0.90) after a graded treadmill test. Ankle-brachial-index after attending SET was 0.86 (1.16$0.62)$ and $0.51(0.29-0.84)$ after graded treadmill test, measured 6-12 months after initial presentation. Ankle-brachial-index was significantly improved after SET in rest $(\mathrm{p}=0.01)$ and after graded treadmill test ( $\mathrm{p}=0.04)$ (fig.3).

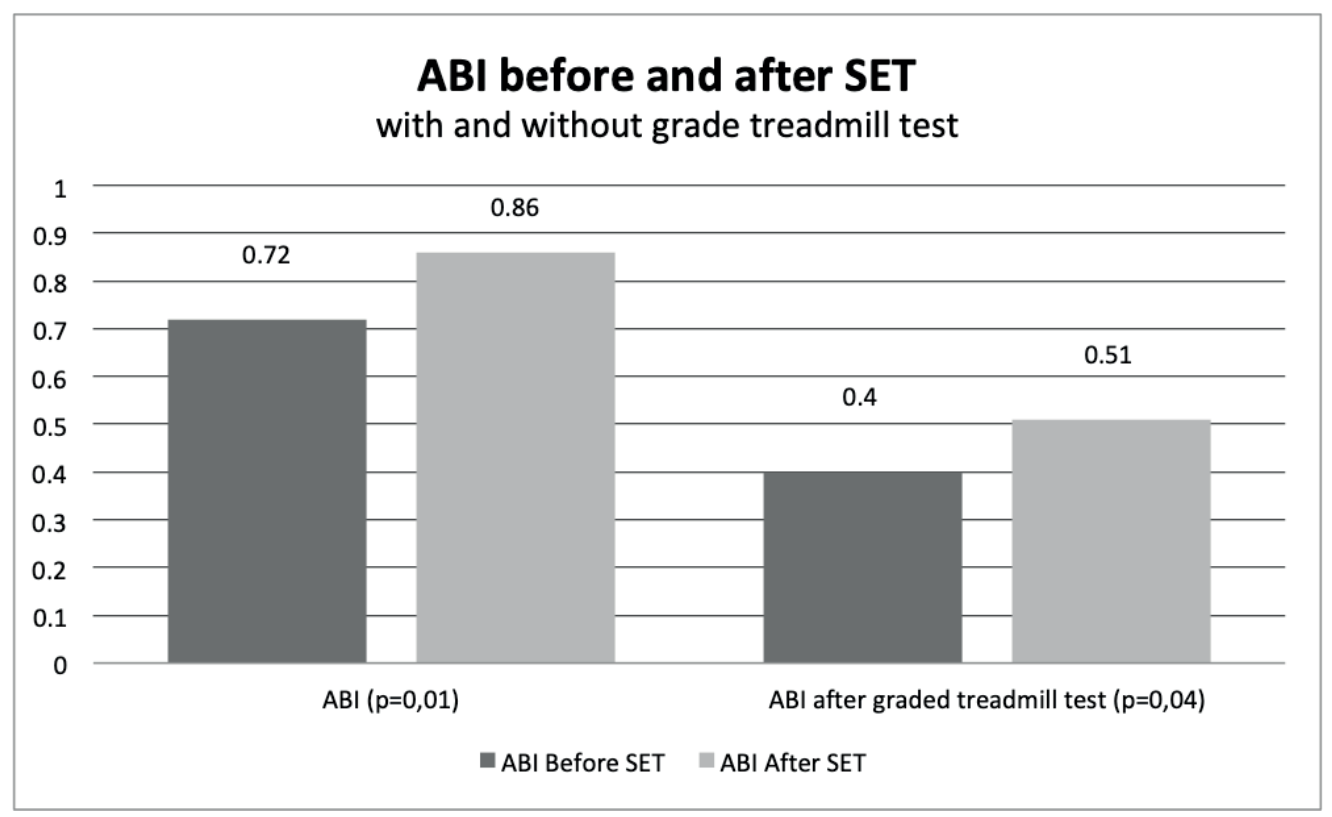

Figure 3. ABI before and after SET

Furthermore, $41 \%$ ( $n=44$ ) of all patients who returned their questionnaire were still active smokers. The reason why patients received SET is clear to $95 \%$ ( $n=97)$. Off all patients $15 \%$ did not understand why they did not qualify for an endovascular procedure.

A reason for premature termination of therapy or not initiating therapy at all was often due to high costs, not covered by basic insurance ( $48 \%$ of all reasons provided, $n=14$ ). Ten percent off all patients stated that they were never referred to a physical therapist for supervised exercise therapy. After initiating SET, 9 patients (8\%) required an endovascular intervention within one year. All of these patients stated to have no self-reported benefit from supervised exercise therapy. 
A significant difference, discriminating between patients who did and those who did not extend PWD after SET, was found for the following determinants: greater knowledge $(\mathrm{p}=0.05)$, positive attitude $(\mathrm{p}=0.03)$ and a low negative attitude $(\mathrm{p}=0.01)$. Patients who were physically more active after having participated in supervised exercise therapy have a more positive attitude $(\mathrm{p}=0.01)$ towards exercise therapy and a higher self-efficacy $(\mathrm{p}=0.05)$, compared to patients not increasing their exercise activities. Logistic regression analysis between patients who extended their walking distance after supervised exercise therapy versus the group who did not extended their walking distance was performed. This analysis indicates that a negative attitude towards exercise therapy to be a significant predictor in the extent of increased COT and PWT ( $\mathrm{p}=0.02)$, with an odds ratio of 3,6.

Anatomical location of stenosis, measured with duplex (significant if stenosis $>70 \%$ ), appears to be relevant in the subjective gain in walking distance after supervised exercise therapy (Table 2). Below the knee (BTK) lesions have the most profit with a gain of 2083 meters, significantly more when compared to femoral (595 meters, $\mathrm{p}=0.03$ ) and multi-level (494 meters, $\mathrm{p}=0.02$ ). However, in the subgroup of patients with aorta-Iliac stenotic lesions no significant difference was found compared to all other anatomic regions (726 meters, resp. $\mathrm{P}=0.1,0.6$ and 0.5 ).

Table 2. Anatomical location of stenosis

\begin{tabular}{ll}
\hline Anatomical location of stenosis & \\
Iliac & $25 \%$ \\
Femoral & $44 \%$ \\
BTK & $7 \%$ \\
Multi-level & $24 \%$ \\
\hline
\end{tabular}

\section{Discussion}

In the current study the authors sought to determine which behavioral determinants are of influence on long-term adherence to SET and improvement of PWD. When participating in supervised exercise therapy, patients with greater knowledge, a positive attitude and a low negative attitude showed better results regarding subjective increase of their PWD. A greater knowledge of peripheral arterial disease and greater awareness of clinical progression of the 
disease might alter behavior and improve attitude and motivation regarding exercise therapy. It is well established that adherence to unsupervised exercise therapy is poor. ${ }^{10}$ Patients with PAD often maintain a sedentary lifestyle to avoid pain, even though disregard to physical exercise will eventually lead to worsening of physical functioning. ${ }^{21,22}$ This is also reflected in a study by Fakhry et al. stating that without proper guidance of supervised exercise therapy, improvement in both COT and PWD is significantly less in noninterventional observation. ${ }^{9,23}$ Furthermore, absence of motivation, socio-economic status, reimbursement issues, low availability and distance to SET location are previously described reasons of low adherence to SET. However, as of January 2017, 37 sessions are reimbursed by basic insurance in the Netherlands, this will possibly contribute to improved adherence. Still, there is a minimum policy excess of $€ 386,-$.

Another reason for low adherence to SET is the perceived information by the patient in the outpatient clinic. Ten percent of patients who were referred for SET did not recall being referred. In other words, information provided in the outpatient clinic is not always interpreted correctly or understood. Despite receiving a referral for SET on paper, being provided with information brochures and follow-up appointments, the medical plan of approach is not always clear to the patient. Furthermore, the low level of education of the study population should be taken into account. The majority of patients received education up to secondary school or lower (65\%). Whereas only $35 \%$ of the patients have an educational level exceeding secondary school. Provision of information and future interventions to improve knowledge need to be comprehensible to the population of interest and should be compatible with level of cognition. Large quantities of complicated information are unlikely to be comprehended. Moreover, complicated educational methods will be unlikely to improve motivation and exercise behavior. Beside obvious physical limitations of PAD, patients also need to adjust mentally to the diagnosis and what this entails. When PAD is established patients are instructed to participate in exercise therapy, whereas no intervention is initiated to address the psychological aspect of coping with PAD. As this study indicates knowledge and awareness should be raised to help the patients understand the disease and possible implications. If the patient is non-compliant to SET attitude and behavior ought to be addressed. Implementing cognitive behavioral therapy could be of great benefit in improvement of adherence to SET. Whereas. McDermott et al. concluded in the GOALS's Trial that a groupmediated cognitive behavioral intervention increased treadmill walking time and physical activity among patient with PAD participating in home-based exercise therapy. ${ }^{24}$ During six months weekly group-meetings with PAD patients and a facilitator where organized to 
increase adherence to walking exercise by offering group support and self-regulatory skills. ${ }^{24}$ This study indicates that improvement of adherence and motivation regarding SET and home-based exercise can be achieved focusing on optimization of knowledge, attitude and self-efficacy. Optimizing these behavioral aspects might increase COT, PWD and acceptance of pain during walking. Brawly et al. found an increase in adherence of a 3 -month home-based walking program after a cognitive- behavioral intervention. ${ }^{25}$ To change attitude and behavior patients need to recognize and accept change is needed and require a willingness to change. It is important that patients formulate their own goals in a process of self-regulation regarding their actions and problematic health behavior. ${ }^{25} \mathrm{To}$ conduct an intervention for the benefit of a patient is clinically senseless when a patient is not willing to change attitude, behavior and actions that might result in regression of mobility. The process of self-regulation is often divided in 5 components, namely: (1) goals comprising desired achievements, (2) self-monitoring of behavior in relation to formulated goals, (3) information and feedback on progress towards their goals, (4) selfevaluation of their advancement, and (5) corrective behavior to optimize progression of the desired goals. ${ }^{25,26}$

A positive attitude and the conviction that complaints of $\mathrm{PAD}$ are reversible with dedicated exercise therapy will stimulate patients and be reflected in perseverance and commitment to (supervised) exercise therapy. Patients' have to believe they can actually succeed in declining or arresting progression of PAD and the possibility of reversing deteriorating mobility is key in successful exercise therapy. In contrast, a patient with negative attitude towards exercise therapy is very unlikely to commit to exercise therapy and thus will not improve on their COT and PWD. Self-efficacy, closely associated with a positive attitude, is the belief that you can actually perform a certain task. In this study we demonstrated that patients who were physically more active after participating in supervise exercise therapy, scored higher on the self-efficacy scale. Confidence in one's own ability to walk long distances is positively associated with walking frequency and duration. ${ }^{21,27,28}$ Selfefficacy is also significantly associated with walking ability in patients with PAD and diabetes mellitus. ${ }^{28}$ Rejeski et al. state that acceptance of pain is a key factor in self-efficacy. If pain was to be accepted by a patient as inevitable when increasing an active lifestyle, improvement of physical function can be expected and might also lead to acceptance of impaired function. ${ }^{21}$ An intervention, regarding self-efficacy and self-regulation in older patients with type 2 diabetes, to increase physical activity resulted in a brief increase in physical activity. Nevertheless, gain in physical activity was not maintained due to low adherence, non-sustained effort and decreasing motivation. Furthermore, increase in 
self-efficacy and self-regulation was not maintained. ${ }^{29}$ Targeted interventions to improve adherence to physical activity can be effective for patients in which increase in physical activity is crucial, but difficult. ${ }^{29}$ However, after completion of a formal program, physical activity related efficacy tends to decline when patients are expected to continue with unsupervised exercise therapy. ${ }^{30}$ When SET is initiated it is important the patient adheres to the therapy. Optimal gain in COT and PWD during SET requires maximum effort of both patient and treating physician. To support patients in their process of behavioral change, improvement of physical activity and adherence to SET, a physician can assist providing information, feedback and assistance in corrective interventions to help patients reach their goal. Interventions to improve adherence have been studied. For instance, as an additional component to exercise, group sessions were simultaneous initiated for discussion and problem-solving. In these sessions the following items were addressed; counselling to improve a patients readiness to change (attitude), education (knowledge), positive reinforcement, setting your own goals (self-efficacy), feedback, an exercise contract, self monitoring using an exercise diary and skill building to help people reach their goals. ${ }^{31}$ These are interventions that should be coincided with the start of an exercise therapy regimen. However, continued outpatient visits and center-based exercise training programs are costly and resource intensive. It takes two months to adapt to new complex behaviors, such as increasing physical activity and requires at least six months to sustain this behavior using more habitual and automatic mechanisms. ${ }^{32,33}$ Commitment by the patient and physician are necessary to realize an successful intervention.

To address the time en cost intensive interventions, novel approaches to assist a patient changing behavior and improve adherence to SET are required. In the era of multimedia distance is relative, as communication is made easy and accessible. In a study by Krpic et al. patients received rehabilitation following stroke in their home setting with the use of multimedia support. With the use of real-time tracking patient's performance was observed. A physiotherapist was able to remotely monitor and adjust training intensity and give instructions with an application on a smartphone. Task performance was greater in the intervention group, whereas clinical effectiveness was not significantly different compared to the in-hospital rehabilitation control group. ${ }^{34}$ Furthermore, homebased rehabilitation was not inferior to hospital-based rehabilitation. Multimedia offer unique advantages to influence health related behavior and the possibility of continued involvement of the treating physician. Multimedia support enables a physician to closely monitor performance, functional progress and creates possibilities to intervene early if necessary. Media delivered interventions can be easily provided to a broad population in 
need of exercise therapy and might be cost-effective. ${ }^{35}$ Strategies to maintain adherence and improve accessibility to exercise therapy should be explored.

This study might be limited due to possible socially acceptable answers given by participants and therefore likely overestimate level of activity and estimated walking distance. Although only 108 of 200 questionnaires where returned, a response rate of $54 \%$ is comparable with previous questionnaire studies among patients with PAD (58\%)..$^{10}$ However, only a few questionnaires were fully completed. This might be due to the confrontation of the consequences of an unhealthy lifestyle and lack of physical exercise. To adjust for this matter, missing data was replaced with a mean of 5 imputations. Furthermore, due to the high number of non-responders $(46 \%)$ it is very likely that patients with negative experiences regarding SET are underrepresented. Therefore, scores testing attitude towards SET could be lower than described in this study. Furthermore, generalizability of this study is estimated to be low. Moreover, this questionnaire was composed using changeable determinants; static facts like socio-economic status are certainly of importance regarding compliance to exercise therapy. As stated earlier, reimbursement issues are often the cause of limited adherence to SET. Furthermore, adherence is difficult to measure retrospectively. To adequately measure adherence a tool requires demonstrated validity and reliability. Whereas validity of the questionnaire was tested with a Cronbach's Alpha $>0.7$, reliability is low. Recalling estimated walking distance is very subjective and not accurate, also the fact that the questionnaire is a single moment of assessment limits the reliability. For future studies it might be useful to use different methods to assess adherence to compensate for weaknesses in measurement methods. For instance, provide participants with the questionnaire before SET end three months after SET and correlate the data with objective data collected by the treadmill, acquired from the physiotherapist.

\section{Conclusion}

This study shows that low adherence to SET can be explained by a lack of knowledge regarding intermittent claudication and absence of positive attitude towards walking behavior. Low adherence to SET will inevitably lead to progression of intermittent claudication. To increase compliance of supervised exercise therapy knowledge, attitude and self-efficacy needs to be addressed. 


\section{References}

1. Sikkink CJ, van Asten WN, van 't Hof MA, van Langen H, van der Vliet JA. Decreased ankle/ brachial indices in relation to morbidity and mortality in patients with peripheral arterial disease. Vasc Med Lond Engl 1997;2(3):169-73. Doi: 10.1177/1358863X9700200302.

2. Criqui $\mathrm{MH}$, Langer RD, Fronek A, Feigelson HS, Klauber MR, McCann TJ, et al. Mortality over a period of 10 years in patients with peripheral arterial disease. $N$ Engl J Med 1992;326(6):381-6. Doi: 10.1056/NEJM199202063260605.

3. McDermott MM, Liu K, Guralnik JM, Criqui MH, Spring B, Tian L, et al. Home-based walking exercise intervention in peripheral artery disease: a randomized clinical trial. JAMA 2013;310(1):57-65. Doi: 10.1001/jama.2013.7231.

4. Hirsch AT, Haskal ZJ, Hertzer NR, Bakal CW, Creager MA, Halperin JL, et al. ACC/AHA 2005 Practice Guidelines for the management of patients with peripheral arterial disease (lower extremity, renal, mesenteric, and abdominal aortic): a collaborative report from the American Association for Vascular Surgery/Society for Vascular Surgery, Society for Cardiovascular Angiography and Interventions, Society for Vascular Medicine and Biology, Society of Interventional Radiology, and the ACC/AHA Task Force on Practice Guidelines (Writing Committee to Develop Guidelines for the Management of Patients With Peripheral Arterial Disease): endorsed by the American Association of Cardiovascular and Pulmonary Rehabilitation; National Heart, Lung, and Blood Institute; Society for Vascular Nursing; TransAtlantic Inter-Society Consensus; and Vascular Disease Foundation. Circulation 2006;113(11):e463-654. Doi: 10.1161/CIRCULATIONAHA.106.174526.

5. Norgren L, Hiatt WR, Dormandy JA, Nehler MR, Harris KA, Fowkes FGR, et al. InterSociety Consensus for the Management of Peripheral Arterial Disease (TASC II). J Vasc Surg 2007;45 Suppl S:S5-67. Doi: 10.1016/j.jvs.2006.12.037.

6. Makris GC, Lattimer CR, Lavida A, Geroulakos G. Availability of Supervised Exercise Programs and the Role of Structured Home-based Exercise in Peripheral Arterial Disease. Eur J Vasc Endovasc Surg 2012;44(6):569-75. Doi: 10.1016/j.ejvs.2012.09.009.

7. Murphy TP, Cutlip DE, Regensteiner JG, Mohler ER, Cohen DJ, Reynolds MR, et al. Supervised Exercise Versus Primary Stenting for Claudication Resulting From Aortoiliac Peripheral Artery Disease: Six-Month Outcomes From the Claudication: Exercise Versus Endoluminal Revascularization (CLEVER) Study. Circulation 2012;125(1):130-9. Doi: 10.1161/CIRCULATIONAHA.111.075770.

8. Frans FA, Bipat S, Reekers JA, Legemate DA, Koelemay MJW. Systematic review of exercise training or percutaneous transluminal angioplasty for intermittent claudication. $\mathrm{Br} J$ Surg 2012;99(1):16-28. Doi: 10.1002/bjs.7656.

9. Fakhry F, van de Luijtgaarden KM, Bax L, den Hoed PT, Hunink MGM, Rouwet EV, et al. Supervised walking therapy in patients with intermittent claudication. J Vasc Surg 2012;56(4):1132-42. Doi: 10.1016/j.jvs.2012.04.046. 
10. Bartelink M-L, Stoffers HEJH, Biesheuvel CJ, Hoes AW. Walking exercise in patients with intermittent claudication. Experience in routine clinical practice. Br J Gen Pract J R Coll Gen Pract 2004;54(500):196-200.

11. Slovinec D'Angelo ME, Pelletier LG, Reid RD, Huta V. The roles of self-efficacy and motivation in the prediction of short- and long-term adherence to exercise among patients with coronary heart disease. Health Psychol Off J Div Health Psychol Am Psychol Assoc 2014;33(11):1344-53. Doi: 10.1037/hea0000094.

12. Martin AM, Woods CB. What sustains long-term adherence to structured physical activity after a cardiac event? J Aging Phys Act 2012;20(2):135-47.

13. Teixeira PJ, Carraça EV, Marques MM, Rutter H, Oppert J-M, De Bourdeaudhuij I, et al. Successful behavior change in obesity interventions in adults: a systematic review of selfregulation mediators. BMC Med 2015;13:84. Doi: 10.1186/s12916-015-0323-6.

14. Vries H de, Mesters I, Steeg H van de, Honing C. The general public's information needs and perceptions regarding hereditary cancer: an application of the Integrated Change Model. Patient Educ Couns 2005;56(2):154-65. Doi: 10.1016/j.pec.2004.01.002.

15. Rogers RW. Cognitive and psychological processes in fear appeals and attitude change: a revised theory of protection motivation. Social psychophysiology-a sourcebook. New York. Guilfort Press; 1983. pp. 153-76.

16. Bandura A, Cioffi D, Taylor CB, Brouillard ME. Perceived self-efficacy in coping with cognitive stressors and opioid activation. J Pers Soc Psychol 1988;55(3):479-88. Doi: 10.1037/00223514.55.3.479.

17. de Vries H, Dijkstra M, Kuhlman P. Self-efficacy: the third factor besides attitude and subjective norm as a predictor of behavioural intentions. Health Educ Res 1988;3(3):273-82. Doi: 10.1093/her/3.3.273.

18. Vries HD, Mudde AN. Predicting stage transitions for smoking cessation applying the attitude-social influence-efficacy model. Psychol Health 1998;13(2):369-85. Doi: 10.1080/08870449808406757.

19. Merry AHH, Teijink JAW, Jongert MWA, Poelgeest A, van der Voort SSEM, Bartelink MEL, et al. KNFG-richtlijn symptomatisch perifeer arterieel vaatlijden 2014.

20. Field A. Discovering statistics using SPSS. Third edition. London: Sage Publications Ltd; 2009.

21. Rejeski WJ, Tian L, Liao Y, McDermott MM. Social cognitive constructs and the promotion of physical activity in patients with peripheral artery disease. J Cardiopulm Rehabil Prev 2008;28(1):65-72. Doi: 10.1097/01.HCR.0000311512.61967.6e.

22. Rejeski WJ, Spring B, Domanchuk K, Tao H, Tian L, Zhao L, et al. A group-mediated, homebased physical activity intervention for patients with peripheral artery disease: effects on social and psychological function. J Transl Med 2014;12:29. Doi: 10.1186/1479-5876-12-29.

23. Fokkenrood HJP, Bendermacher BLW, Lauret GJ, Willigendael EM, Prins MH, Teijink JAW. Supervised exercise therapy versus non-supervised exercise therapy for intermittent claudication. Cochrane Database Syst Rev 2013;8:CD005263. Doi: 10.1002/14651858. CD005263.pub3. 
24. McDermott MM, Guralnik JM, Criqui MH, Ferrucci L, Liu K, Spring B, et al. Unsupervised exercise and mobility loss in peripheral artery disease: a randomized controlled trial. $\mathrm{J} \mathrm{Am}$ Heart Assoc 2015;4(5). Doi: 10.1161/JAHA.114.001659.

25. Brawley LR, Rejeski WJ, King AC. Promoting physical activity for older adults: the challenges for changing behavior. Am J Prev Med 2003;25(3 Suppl 2):172-83.

26. Barone DF, Maddux JE, Snyder CR. Social Cognitive Psychology. Boston, MA: Springer US; 1997.

27. Caldieraro-Bentley AJ, Andrews JO. An integrative review: Application of self-efficacy instruments for walking in populations with peripheral arterial disease. J Vasc Nurs 2013;31(3):118-30. Doi: 10.1016/j.jvn.2013.01.002.

28. Collins TC, Lunos S, Ahluwalia JS. Self-efficacy is associated with walking ability in persons with diabetes mellitus and peripheral arterial disease. Vasc Med 2010;15(3):189-95. Doi: 10.1177/1358863X10362604.

29. Olson EA, McAuley E. Impact of a brief intervention on self-regulation, self-efficacy and physical activity in older adults with type 2 diabetes. J Behav Med 2015;38(6):886-98. Doi: 10.1007/s10865-015-9660-3.

30. McAuley E, Mailey EL, Mullen SP, Szabo AN, Wójcicki TR, White SM, et al. Growth trajectories of exercise self-efficacy in older adults: influence of measures and initial status. Health Psychol Off J Div Health Psychol Am Psychol Assoc 2011;30(1):75-83. Doi: 10.1037/ a0021567.

31. Jorden JL, Holden MA, Mason EEJ, Foster NE. Interventions to improve adherence to exercise for chronic musculoskeletal pain in adults (Review). Cochrane Database of Systematic Reviews 2010, Issue 1. [DOI: 10.1002/14651858.CD005956.pub2]

32. Lally P, van Jaarsveld CHM, Potts HWW, Wardle J. How are habits formed: Modelling habit formation in the real world. Eur J Soc Psychol 2010;40(6):998-1009. Doi: 10.1002/ejsp.674.

33. Dishman RK. Compliance/adherence in health-related exercise. Health Psychol 1982;1(3):23767. Doi: 10.1037/0278-6133.1.3.237.

34. Krpič A, Savanović A, Cikajlo I. Telerehabilitation: remote multimedia-supported assistance and mobile monitoring of balance training outcomes can facilitate the clinical staff's effort. Int J Rehabil Res Int Z Für Rehabil Rev Int Rech Réadapt 2013;36(2):162-71. Doi: 10.1097/ MRR.06013e32835dd63b.

35. Gothe NP, Wójcicki TR, Olson EA, Fanning J, Awick E, Chung HD, et al. Physical activity levels and patterns in older adults: the influence of a DVD-based exercise program. J Behav Med 2015;38(1):91-7. Doi: 10.1007/s10865-014-9581-6. 
снатіве, General Discussion

\& Summary 
In summary, this thesis has given an insight in post contrast acute kidney injury (PC-AKI), long-term effects of iodine contrast on kidney function and exposure levels to ionizing radiation in endovascular treatment of the lower extremities. Furthermore, it has provided an alternative technique in the treatment of iliac TASC II A and B lesions, and a way to prevent PC-AKI by inducing furosemide forced diuresis with matched hydration. Lastly, behavioural determinants, influencing adherence in supervised exercise therapy (SET), are established.

The second chapter studied the long-term effects of iodine contrast on renal function in patients newly presenting to the vascular outpatient clinic with Rutherford class II or III peripheral arterial disease. Patients were treated with either supervised exercise therapy or endovascular interventions. Changes in estimated glomerular filtration rate (eGFR) after one year were compared between the two treatment groups. Results showed clinically relevant and long-term loss of kidney function after endovascular procedures for peripheral arterial disease.

In continuation of the previous chapter, chapter three studied the long-term effects of iodine contrast on renal function in patients with critical limb ischemia (Rutherford class IV-VI) receiving either an endovascular intervention or open surgery. eGFR after one year was compared, as well as the percentage of patient experiencing fast renal decline $(>4$ $\mathrm{ml} / \mathrm{min}$ within one year). Results revealed permanent long-term loss of kidney function after endovascular procedures for critical limb ischemia. This loss of renal function was significantly greater when compared to patients treated with open surgery.

Chapter four gives an insight regarding sustained ionizing radiation exposure in the endovascular treatment of patients with symptomatic PAD. In this single centre observational cohort study, dose area product (DAP), peak skin dose (PSD), fluoroscopy time and volume of contrast media were measured or calculated. Results were stratified according patient demographics and anatomical location. This chapter shows that even though fluoroscopy time is shorter, aorto-iliac interventions result in greater radiation exposure when compared to femoropopliteal or infra-popliteal interventions.

In the search of a preventive measure against PC-AKI and ionizing radiation effects, a randomized controlled trial was constructed in chapter five. A relatively novel application of a long existing imaging technique was used, treating TASC II A/B iliac lesions with duplex. Technical success was evaluated comparing Duplex guided PTA (DuPTA) with 
conventional PTA. Results showed duplex-guided PTA to be a feasible and non-inferior alternative to conventional PTA in the treatment of PAD on the iliac anatomic level. Furthermore, a significant greater reduction in peak systolic velocity was achieved using DuPTA.

The results in chapter three showed 19\% AKI in patients treated endovascularly for PAD Rutherford IV-VI. To pursue these acute effects of contrast on renal function, Chapter six compared short- and long-term renal function after duplex guided percutaneous transluminal angioplasty (DuPTA) and conventional (contrast mediated) PTA in patients with iliac TASC II A/B lesions. However, no significant difference in renal decline was observed. Furthermore, none of the patients developed a significant reduction in eGFR.

Endovascular treatment of patients with PAD is often complicated due to comorbidities, such as chronic kidney disease (CKD), making them more susceptible to PC-AKI. To address this problem, patients with CKD receive pre- and post-hydration. However, fluid volume administered is often too low to wield any real protective effect, out of fear of fluid overload or pulmonary oedema. Chapter 7 entails a study protocol regarding the Renalguard, enabling us to match fluid hydration after furosemide induced diuresis, while performing endovascular revascularization. This enables us to achieve an increased and steady renalperfusion, without risking fluid overload, pulmonary oedema or volume depletion. Furthermore, using the study protocol in chapter 7 , we will sample urine biomarkers 4 hours post procedure to evaluate whether these biomarkers are a valid alternative in diagnosing PC-AKI, whereas serum creatine needs 48-72 hours to show a significant rise, and therefore delay in diagnosis.

Whereas the previous chapters identify possible side effects of endovascular surgery and study alternative techniques and measures, the best approach is to prevent rather than to cure. Patients newly presenting to the outpatient clinic with PAD are often confronted with intermittent claudication. The first step in treatment, based on international guidelines, is to prescribe supervised exercise therapy (SET). Even though studies show significant increase in pain free walking distance and claudication onset time, adherence to prescribed SET is low. Chapter $\mathbf{8}$ studies which behavioural determinants are involved, explaining low adherence to SET. A questionnaire was constructed, using the I-CHANGE model, and was sent to 200 patients who were prescribed SET. Patients who increased their walking distance after SET have significantly greater knowledge, positive attitude and lower negative attitude towards SET. Increasing the determinants knowledge, attitude 
and self-efficacy will improve adherence to SET and enhances claudication onset time. Furthermore, resorting to surgical treatment might be less often necessary.

\section{Discussion renal function}

Chapter 3 showed an increased incidence of PC-AKI after endovascular intervention with iodine contrast, treating patients with critical limb ischemia. Interestingly, these results do not correlate to the findings in chapter 6 , whereas patients treated endovascularly for intermittent claudication did not show any signs of PC-AKI. However, Sigterman et al. described an 13\% incidence of PC-AKI in patients treated with PTA, regardless of prior renal function and disease progression (Rutherford II-VI ). ${ }^{1}$ Although PC-AKI was not diagnosed after endovascular treatment in patients with intermittent claudication in chapter 6 , even slight increases in serum creatinine are associated with increased in hospital mortality and acceleration to end-stage renal failure requiring dialysis. ${ }^{2-5}$ Furthermore, incidence and prevalence of PC-AKI are rising 5-8\% annually. ${ }^{6}$ Fortunately, when recognized, AKI is often effectively treated with supportive therapy, however there remains an increased risk of long-term kidney failure. ${ }^{7}$

Chapter two of this thesis showed a significant decrease in long-term kidney function in patients receiving an endovascular intervention of the lower extremities, when compared to patients receiving supervised exercise therapy (SET). Furthermore, 64\% experienced fast renal decline $\left(<4 \mathrm{ml} \mathrm{min} / 1.73 \mathrm{~m}^{2}\right)$ within one year. The risk of fast renal decline was 11 times as high when compared to the control group. Chapter 3 studied eGFR decline in patients with critical limb ischemia (CLI), comparing patients treated with an endovascular intervention vs open surgery. $77 \%$ of patients treated endovascularly showed fast renal decline after one-year, compared to $54 \%$ in the open surgery group. It should be noted however, that patients with CLI have an increased incidence of CKD, CHF, diabetes, hypertension, coronary artery disease, all contributing to increased deterioration in renal function. Furthermore, revascularization of ischemic extremities create multiple systemic problems impacting renal function, such as metabolic acidosis, hyperkalaemia, myoglobinuria. ${ }^{8}$

Previous study by Arora et al., showed an increased risk of long-term CKD and mortality in patients who develop AKI after revascularization of the lower extremities. ${ }^{9}$ Chapter 6 does underline the result of fast renal decline after endovascular intervention, one-year post procedure. Noteworthy is the fact that $48 \%$ of the patients treated with duplex guided PTA (DuPTA) in chapter 6 also had fast renal decline. Therefore, the cause 
of AKI can not solely be assigned to iodine contrast. It can be argued that dislodging of debris due to manipulation of guidewires near the ostia of the renal arteries might play an important role. To support this theory, a study by Cortese et al., showed that the incidence of AKI in coronary interventions was significantly lower after transradial approach when compared to transfemoral approach. ${ }^{10}$ This might be due to the passing of the guidewire through the abdominal aorta and past the renal arteries. Furthermore, Keeley at al., showed that 50 percent of guiding catheters contained plaque debris after femoral approach performing percutaneous revascularization. ${ }^{11}$ However, to differentiate between CIN and artheroembolic renal disease (AERD) is difficult, whereas the only way to diagnose AERD is through renal biopsy or post mortem autopsy. Together these results suggest that deterioration of renal function post endovascular intervention is most certainly multifactorial, including comorbidities, nephrotoxic medication, iodine contrast, manipulation of guidewires, ischemia of the leg, age etc. It is therefore of utmost importance to monitor renal function post endovascular intervention, especially in highrisk patients.

\section{Discussion radiation exposure}

Ionizing radiation is known to be potentially harmful for both patient and clinical staff. Regrettably, due to the progressive nature of peripheral arterial disease (PAD), patients are often repeatedly exposed, increasing stochastic effect and chance of malignancies. Therefor international guidelines are formulated and clinics are stimulated to work with the ALARA principles, "as low as reasonably achievable". ${ }^{12}$ Furthermore, equipment and contrast agents are improved continuously to minimalize potential detrimental effects. To quantitate the amount of radiation dosage used per procedure, dose area product (DAP) is frequently used in literature. DAP correlates best to the area exposed, whereas peak skin dose (PSD) is an estimate of the DAP and less reliable because of c-bow angulation and shifting focus of ionizing beam. To protect the patients against detrimental effects, the national council on radiation protection and measurements (NCRP) guidelines were drafted to establish maximal radiation dosage per procedure. ${ }^{13,14}$ Peak skin dose (PSD) should not exceed $3 \mathrm{~Gy}$, whereas DAP is capped at $500 \mathrm{~Gy}^{*} \mathrm{~cm}^{2} .{ }^{16}$ In chapter 4 of this thesis both DAP as PSD stayed well under the maximal radiation dose in endovascular treatment of the lower extremities. Mean DAP was $142 \pm 103$ in aortoiliac procedures. Compared to all other anatomical locations (femoral, below-the-knee (BTK) and multilevel) DAP was significantly higher. These results are supported by literature. ${ }^{13,15-17}$ Goldsweig et al. 
published a study identifying 17.174 patients from The National Cardiovascular Data Registry Peripheral Vascular Intervention Registry treated endovascularly for atherosclerotic disease of the lower extremities. Interventions treating acute limb ischemia and aneurysm repair where excluded. DAP levels on aortoiliac procedures $(252 \pm 294.4)$ where a lot higher when compared to our results, even though there are no significant differences in patient characteristics. Patients in our study were treated several years before the study by Goldsweig et al., distribution in severity of PAD (Rutherford classification) was similar. In both this thesis and similar studies, BMI is identified as an independent predictor of increased DAP. ${ }^{13,15,16,18}$ Greater DAP in pelvic procedures and in obese patients can be explained by the automatic exposure control of the $\mathrm{x}$-ray system to maintain brightness and contrast. Increased photon energy is required due to added mass between skin and artery, absorbing and scattering the fluoroscopic beam. ${ }^{13,16}$ Furthermore, BTK lesions can be treated with a narrower field of view, limiting DAP. Also contributing to a higher DAP are the retrograde up-and-over procedures, were the contralateral leg is accessed and the aortoiliac-bifurcation needs to be crossed, leading to higher radiation dosage whereas the added tissue mass of the abdomen and pelvis needs to penetrated by the ionizing radiation. ${ }^{13,15}$ Formulating the plan of approach in the endovascular treatment of peripheral arterial disease needs to take in consideration how to access the lesion, minimalizing DAP and fluoroscopy time.

\section{Discussion novel treatment strategies}

One of the causes of AKI are nephrotoxic agents, such as iodine contrast, used in endovascular procedures. Patients with chronic kidney disease (CKD) are especially susceptive to the toxic properties of iodine contrast, causing contrast induced nephropathy (CIN). ${ }^{19} \mathrm{To}$ anticipate on the potential damage, patients with $\mathrm{CKD}$ requiring an endovascular intervention are treated with pre- and post-hydration using saline for volume expansion to diminish possible toxic effects. ${ }^{17,23}$ Previous guidelines advised pre- and post-hydration in patients with an eGFR $<45 \mathrm{ml} / \mathrm{min}$ or eGFR $<60$ with additional risk factors (diabetes mellitus, heart failure, age $>75$ years, hypotension, and anaemia). ${ }^{21}$ The AMACING trial showed that patients with eGFR $>30 \mathrm{ml} / \mathrm{min} / 1.73 \mathrm{~m}^{2}$, without additional risk factors have no increased risk of developing CIN when not receiving volume expansion. ${ }^{22}$ In this thesis however, patients were treated with pre- and post-hydration therapy based on previous guidelines. ${ }^{23,24}$ 
Chapter 5 entails an RCT comparing conventional PTA of the iliac arteries to duplex guided PTA (DuPTA) in TASC II A/B lesions. Whereas conventional PTA relies on the use of iodine contrast and ionizing radiation, DuPTA enables us to perform an PTA without potentially causing CIN and/or negative side effects of ionizing radiation. Previous studies have demonstrated DuPTA as a feasible alternative, however no evidence was available regarding the endovascular treatment of iliac lesions. It was argued that DuPTA would not be possible of the aortoiliac arteries due to bowel gases and increased body mass in obese patients. ${ }^{25}$ However, in this thesis we did not experience any problems regarding duplex imaging. The goal of this study was to prove DuPTA to be non-inferior to conventional PTA and provide a treatment option for patients in need of an iliac endovascular intervention but with a contra-indication due to $\mathrm{CKD}$ or hypersensitivity to iodine contrast. Our results demonstrated that in comparison with conventional PTA, DuPTA has similar success in passing the stenotic lesion with a guidewire and performing the PTA. Furthermore, significant greater improvement in peak systolic velocity (PSV) was achieved in the DuPTA group. Another benefit of DuPTA is the direct hemodynamic feedback post-procedure to evaluate possible recoil or flow-limiting dissection, whereas this is not as reliable in conventional PTA's. Completion angiography is only accurate in $76 \%$ of PTA's. ${ }^{25}$ However, it should be mentioned that $11 \%$ of the patients treated with DuPTA still required contrast to successfully treat the lesion. This was partially explained by progression of the stenotic lesion to complete occlusion at the time of intervention. In other literature, using CO2 as an alternative to iodine contrast, the use of iodine contrast to successfully perform the procedure was more often required. In two studies by Mendes et al., treating iliac TASC II A/B lesions, $21-36 \%$ of the patients required additional iodine contrast, whereas $\mathrm{CO} 2$ was not sufficient. ${ }^{26,27}$

As was previously mentioned. Patients with an eGFR $<45 \mathrm{ml} / \mathrm{min} .1 .73 \mathrm{~m}^{2}$ or eGFR $<60$ $\mathrm{ml} / \mathrm{min} .1 .73 \mathrm{~m}^{2}$ with additional risk factors (DM, HF, old age, anaemia) receive pre- and post-hydration therapy. Patients receive $3-4 \mathrm{ml} / \mathrm{kg} / \mathrm{h}$ of $0,9 \% \mathrm{NaCl}$ intravenously 4 hours pre- and 4hours post intervention. Patients with CHF, at risk of fluid overload, or eGFR $<30 \mathrm{ml} / \mathrm{min} .1 .73 \mathrm{~m}^{2}$ receive $1 \mathrm{ml} / \mathrm{kg} / \mathrm{h} 12$ hours pre- and post-procedure. Preventing dehydration and increasing diuresis are known to protect against PC-AKI. ${ }^{4,28-31}$ The volume of administered fluids in the prevention of PC-AKI is often too low to adequately diminish nephrotoxic effects of iodine contrast, because of fear of fluid overload and pulmonary oedema. ${ }^{31}$ Furosemide forced diuresis is studied, however a mismatch between administered fluid and furosemide can cause volume depletion and therefore increase concentration in the kidneys, increasing the chance of PC-AKI. ${ }^{32-35}$ Chapter 7 is a study protocol regarding 
the use of the Renalguard to match volume of fluid administration too furosemide forced diuretic output. The goal is to create diuretic output of $>300 \mathrm{ml} / \mathrm{h}$, diluting and limit exposure time of the nephrotoxic iodine contrast in the kidneys. The Renalguard is a safe and effective tool in maintaining intravenous volume without instigating volume depletion or overload. ${ }^{30}$ Moreover, Marenzi et al., demonstrated a reduction of PC-AKI in $74 \%$ of patients receiving iodine contrast for diagnostic purposes. ${ }^{30} \mathrm{Up}$ to date, no literature is available using furosemide forced diuresis with matched hydration in patients with treated endovascularly for symptomatic PAD.

Chapter 7 also evaluated the current diagnostic tool for AKI using serum creatinine levels 48-72 hours postintervention and researching urine biomarkers as an alternative in early detection of AKI. Serum creatinine rises slowly detecting PC-AKI and is therefore an inadequate tool. ${ }^{36-38}$ Furthermore, detection of changes in serum creatine is only possible when $>50 \%$ of eGFR is lost. ${ }^{39,40}$ Patients treated endovascularly are usually discharged within 24 hours post intervention and are instructed to return for serum creatine levels after 2-3 days. However, in our experience patients often dismiss this instruction making it possible to miss potential PC-AKI. Furthermore, hydration prophylaxis requires extended hospitalization, stretching healthcare budgets and hospital logistics. ${ }^{22}$ To address this problem, chapter 7 in this thesis proposes to study the effect of an endovascular intervention on urine biomarkers, NGAL, KIM-1 and IL-18. Literature identified these urine biomarkers (among others) to detect AKI in an early stage. ${ }^{37,38,41,42}$ Rise in NGAL concentration is greatest 4-6 hours post intervention, with an increase up to 25 times compared with baseline value. ${ }^{38}$ This thesis hypotheses that the detection of urine biomarkers will enable us to detect and anticipate PC-AKI early on post endovascular intervention.

\section{Discussion supervised exercise therapy}

"Prevention is better than cure". The previous chapters in this thesis identify, address and provide solutions to possible side effects of endovascular interventions in patients with PAD. First presentation of patients with PAD, in the outpatient clinic, is often with intermittent claudication. Before endovascular or surgical treatments are explored, patients are prescribed supervised exercise therapy, as is recommended by international guidelines. ${ }^{43}$ Literature shows that after 6 months of SET functional performance, prolonging claudication onset time (COT) and peak walking time (PWT), are considerably 
improved. ${ }^{43-46}$ Regrettably, adherence to SET is low due to imbursement issues, limited availability and absence of motivation. ${ }^{45,47}$ Sixty-eighth percent of patients prescribed SET do actually participate. ${ }^{48}$ This is comparable to the findings in this thesis, with $79 \%$ of the patients participating to SET. Chapter 8 addresses the low adherence to SET and tries to determine which behavioural aspects are involved. A questionnaire was constructed using the I-CHANGE model for explaining motivational an behavioural change. ${ }^{49}$ It appeared patients with greater knowledge, positive attitude and low negative attitude have a greater subjective improvement in peak walking distance (PWD). Furthermore, proper guidance of SET improves both COT and PWD to a greater extend. ${ }^{47}$ Chapter 8 also state that knowledge and awareness should be raised to help patients understand the aetiology of and lifestyle choices contributing to the disease, make them aware what their behaviour instigates and the possible implications this entails. Furthermore, knowledge, attitude and self-efficacy need to be addressed to improve adherence to SET. Whereas confidence in one's own ability to walk long distances is positively associated with walking frequency PWD. ${ }^{50-52}$ 


\section{References}

1. Sigterman TA, Krasznai AG, Snoeijs MG, Heijboer R, Schurink GWH, Bouwman LH. Contrast Induced Nephropathy and Long-term Renal Decline After Percutaneous Transluminal Angioplasty for Symptomatic Peripheral Arterial Disease. Eur J Vasc Endovasc Surg Off J Eur Soc Vasc Surg. 2015 Oct 9;

2. Weisbord SD, Chen H, Stone RA, Kip KE, Fine MJ, Saul MI, et al. Associations of increases in serum creatinine with mortality and length of hospital stay after coronary angiography. $J$ Am Soc Nephrol JASN. 2006 Oct;17(10):2871-7.

3. Levy EM, Viscoli CM, Horwitz RI. The effect of acute renal failure on mortality. A cohort analysis. JAMA. 1996 May 15;275(19):1489-94.

4. Hoste EAJ, De Corte W. Clinical consequences of acute kidney injury. Contrib Nephrol. 2011;174:56-64.

5. Rihal CS, Textor SC, Grill DE, Berger PB, Ting HH, Best PJ, et al. Incidence and prognostic importance of acute renal failure after percutaneous coronary intervention. Circulation. 2002 May 14;105(19):2259-64.

6. Parfrey PS, Griffiths SM, Barrett BJ, Paul MD, Genge M, Withers J, et al. Contrast materialinduced renal failure in patients with diabetes mellitus, renal insufficiency, or both. A prospective controlled study. N Engl J Med. 1989 Jan 19;320(3):143-9.

7. Waikar SS, Winkelmayer WC. Chronic on acute renal failure: long-term implications of severe acute kidney injury. JAMA. 2009 Sep 16;302(11):1227-9.

8. Morsey H, Aslam M, Standfield N. Patients with critical ischemia of the lower limb are at risk of developing kidney dysfunction. Am J Surg. 2003 Apr;185(4):360-3.

9. Arora P, Davari-Farid S, Pourafkari L, Gupta A, Dosluoglu HH, Nader ND. The effect of acute kidney injury after revascularization on the development of chronic kidney disease and mortality in patients with chronic limb ischemia. J Vasc Surg. 2015 Mar;61(3):720-7.

10. Cortese B, Sciahbasi A, Sebik R, Rigattieri S, Alonzo A, Silva-Orrego P, et al. Comparison of risk of acute kidney injury after primary percutaneous coronary interventions with the transradial approach versus the transfemoral approach (from the PRIPITENA urban registry). Am J Cardiol. 2014 Sep 15;114(6):820-5.

11. Keeley EC, Grines CL. Scraping of aortic debris by coronary guiding catheters: a prospective evaluation of 1,000 cases. J Am Coll Cardiol. 1998 Dec;32(7):1861-5.

12. Hendee WR, Edwards FM. ALARA and an integrated approach to radiation protection. Semin Nucl Med. 1986 Apr;16(2):142-50.

13. Goldsweig AM, Kennedy KF, Abbott JD, Jones WS, Velagapudi P, Rutar FJ, et al. Patient Radiation Dosage During Lower Extremity Endovascular Intervention. JACC Cardiovasc Interv. 2019 11;12(5):473-80.

14. Measurements NC on RP and. Radiation Dose Management for Fluoroscopically Guided Interventional Medical Prcoedures. [Internet]. Bethesda: National Council on Radiation 
Protection and Measurements (NCRP); 2010 [cited 2020 Jun 9]. Available from: http:// public.ebookcentral.proquest.com/choice/publicfullrecord.aspx?p=3382409

15. Segal E, Weinberg I, Leichter I, Klimov A, Giri J, Bloom AI. Patient radiation exposure during percutaneous endovascular revascularization of the lower extremity. J Vasc Surg. 2013 Dec;58(6):1556-62.

16. Boc V, Boc A, Zdešar U, Blinc A. Patients' radiation doses during percutaneous endovascular procedures in arteries of the lower limbs. VASA Z Gefasskrankheiten. 2019 Mar;48(2):167-74.

17. Majewska N, Blaszak MA, Juszkat R, Frankiewicz M, Makalowski M, Majewski W. Patients' radiation doses during the implantation of stents in carotid, renal, iliac, femoral and popliteal arteries. Eur J Vasc Endovasc Surg OffJ Eur Soc Vasc Surg. 2011 Mar;41(3):372-7.

18. Ketteler ER, Brown KR. Radiation exposure in endovascular procedures. J Vasc Surg. 2011 Jan;53(1 Suppl):35S-38S.

19. Nash K, Hafeez A, Hou S. Hospital-acquired renal insufficiency. Am J Kidney Dis Off J Natl Kidney Found. 2002 May;39(5):930-6.

20. National Clinical Guideline Centre (UK). Acute Kidney Injury: Prevention, Detection and Management Up to the Point of Renal Replacement Therapy [Internet]. London: Royal College of Physicians (UK); 2013 [cited 2020 Jun 10]. (National Institute for Health and Clinical Excellence: Guidance). Available from: http://www.ncbi.nlm.nih.gov/books/NBK247665/

21. Stacul F, van der Molen AJ, Reimer P, Webb JAW, Thomsen HS, Morcos SK, et al. Contrast induced nephropathy: updated ESUR Contrast Media Safety Committee guidelines. Eur Radiol. 2011 Dec;21(12):2527-41.

22. Nijssen EC, Rennenberg RJ, Nelemans PJ, Essers BA, Janssen MM, Vermeeren MA, et al. Prophylactic hydration to protect renal function from intravascular iodinated contrast material in patients at high risk of contrast-induced nephropathy (AMACING): a prospective, randomised, phase 3, controlled, open-label, non-inferiority trial. The Lancet. 2017 Apr;389(10076):1312-22.

23. Khwaja A. KDIGO clinical practice guidelines for acute kidney injury. Nephron Clin Pract. 2012;120(4):c179-184.

24. Ad-hoc working group of ERBP, Fliser D, Laville M, Covic A, Fouque D, Vanholder R, et al. A European Renal Best Practice (ERBP) position statement on the Kidney Disease Improving Global Outcomes (KDIGO) clinical practice guidelines on acute kidney injury: part 1: definitions, conservative management and contrast-induced nephropathy. Nephrol Dial Transplant Off Publ Eur Dial Transpl Assoc - Eur Ren Assoc. 2012 Dec;27(12):4263-72.

25. Ramaswami G, Al-Kutoubi A, Nicolaides AN, Dhanjil S, Vilkomerson D, Ferrara-Ryan M, et al. Angioplasty of lower limb arterial stenoses under ultrasound guidance: single-center experience. J Endovasc Surg Off J Int Soc Endovasc Surg. 1999 Feb;6(1):52-8.

26. Mendes C de A, Martins A de A, Teivelis MP, Kuzniec S, Varella AYM, Fioranelli A, et al. Carbon dioxide contrast medium for endovascular treatment of ilio-femoral occlusive disease. Clin Sao Paulo Braz. 2015 Oct;70(10):675-9.

27. de Almeida Mendes C, de Arruda Martins A, Teivelis MP, Kuzniec S, Nishinari K, Krutman M, 
et al. Carbon dioxide is a cost-effective contrast medium to guide revascularization of TASC A and TASC B femoropopliteal occlusive disease. Ann Vasc Surg. 2014 Aug;28(6):1473-8.

28. Trivedi HS, Moore H, Nasr S, Aggarwal K, Agrawal A, Goel P, et al. A randomized prospective trial to assess the role of saline hydration on the development of contrast nephrotoxicity. Nephron Clin Pract. 2003 Jan;93(1):C29-34.

29. Mueller C, Buerkle G, Buettner HJ, Petersen J, Perruchoud AP, Eriksson U, et al. Prevention of contrast media-associated nephropathy: randomized comparison of 2 hydration regimens in 1620 patients undergoing coronary angioplasty. Arch Intern Med. 2002 Feb 11;162(3):329-36.

30. Marenzi G, Ferrari C, Marana I, Assanelli E, De Metrio M, Teruzzi G, et al. Prevention of Contrast Nephropathy by Furosemide With Matched Hydration. JACC Cardiovasc Interv. 2012 Jan; (1):90-7.

31. Pannu N, Wiebe N, Tonelli M, Alberta Kidney Disease Network for the. Prophylaxis Strategies for Contrast-Induced Nephropathy. JAMA. 2006 Jun 21;295(23):2765.

32. Weisberg LS, Kurnik PB, Kurnik BR. Risk of radiocontrast nephropathy in patients with and without diabetes mellitus. Kidney Int. 1994 Jan;45(1):259-65.

33. Weinstein JM, Heyman S, Brezis M. Potential deleterious effect of furosemide in radiocontrast nephropathy. Nephron. 1992;62(4):413-5.

34. Solomon R, Werner C, Mann D, D'Elia J, Silva P. Effects of saline, mannitol, and furosemide to prevent acute decreases in renal function induced by radiocontrast agents. $N$ Engl J Med. 1994 Nov 24;331(21):1416-20.

35. Stevens MA, McCullough PA, Tobin KJ, Speck JP, Westveer DC, Guido-Allen DA, et al. A prospective randomized trial of prevention measures in patients at high risk for contrast nephropathy: results of the P.R.I.N.C.E. Study. Prevention of Radiocontrast Induced Nephropathy Clinical Evaluation. J Am Coll Cardiol. 1999 Feb;33(2):403-11.

36. Erdbruegger U, Palevsky PM, Sheridan AM. Etiology and diagnosis of prerenal disease and acute tubular necrosis in acute kidney injury (acute renal failure). Uptodate.com; 2014.

37. Erdbruegger U, Palevsky PM, Sheridan AM. Investigational biomarkers and the evaluation of acute tubular necrosis. Uptodate.com; 2014.

38. Bennett M, Dent CL, Ma Q, Dastrala S, Grenier F, Workman R, et al. Urine NGAL predicts severity of acute kidney injury after cardiac surgery: a prospective study. Clin J Am Soc Nephrol CJASN. 2008 May;3(3):665-73.

39. Delanaye P, Cavalier E, Pottel H. Serum Creatinine: Not So Simple! Nephron. 2017;136(4):3028.

40. Ronco C, Bellomo R, Kellum J. Understanding renal functional reserve. Intensive Care Med. 2017;43(6):917-20.

41. Cruz DN, Bagshaw SM, Maisel A, Lewington A, Thadhani R, Chakravarthi R, et al. Use of biomarkers to assess prognosis and guide management of patients with acute kidney injury. Contrib Nephrol. 2013;182:45-64.

42. Ronco C, Legrand M, Goldstein SL, Hur M, Tran N, Howell EC, et al. Neutrophil gelatinaseassociated lipocalin: ready for routine clinical use? An international perspective. Blood Purif. 
2014;37(4):271-85.

43. Hirsch AT, Haskal ZJ, Hertzer NR, Bakal CW, Creager MA, Halperin JL, et al. ACC/ AHA 2005 Practice Guidelines for the management of patients with peripheral arterial disease (lower extremity, renal, mesenteric, and abdominal aortic): a collaborative report from the American Association for Vascular Surgery/Society for Vascular Surgery, Society for Cardiovascular Angiography and Interventions, Society for Vascular Medicine and Biology, Society of Interventional Radiology, and the ACC/AHA Task Force on Practice Guidelines (Writing Committee to Develop Guidelines for the Management of Patients With Peripheral Arterial Disease): endorsed by the American Association of Cardiovascular and Pulmonary Rehabilitation; National Heart, Lung, and Blood Institute; Society for Vascular Nursing; TransAtlantic Inter-Society Consensus; and Vascular Disease Foundation. Circulation. 2006 Mar 21;113(11):e463-654.

44. Norgren L, Hiatt WR, Dormandy JA, Nehler MR, Harris KA, Fowkes FGR, et al. InterSociety Consensus for the Management of Peripheral Arterial Disease (TASC II). J Vasc Surg. 2007 Jan; 45 Suppl S:S5-67.

45. Makris GC, Lattimer CR, Lavida A, Geroulakos G. Availability of Supervised Exercise Programs and the Role of Structured Home-based Exercise in Peripheral Arterial Disease. Eur J Vasc Endovasc Surg. 2012 Dec;44(6):569-75.

46. Murphy TP, Cutlip DE, Regensteiner JG, Mohler ER, Cohen DJ, Reynolds MR, et al. Supervised Exercise Versus Primary Stenting for Claudication Resulting From Aortoiliac Peripheral Artery Disease: Six-Month Outcomes From the Claudication: Exercise Versus Endoluminal Revascularization (CLEVER) Study. Circulation. 2012 Jan 3;125(1):130-9.

47. Fakhry F, van de Luijtgaarden KM, Bax L, den Hoed PT, Hunink MGM, Rouwet EV, et al. Supervised walking therapy in patients with intermittent claudication. J Vasc Surg. 2012 Oct;56(4):1132-42.

48. Bartelink M-L, Stoffers HEJH, Biesheuvel CJ, Hoes AW. Walking exercise in patients with intermittent claudication. Experience in routine clinical practice. Br J Gen Pract J R Coll Gen Pract. 2004 Mar;54(500):196-200.

49. Vries $\mathrm{H}$ de, Mesters I, Steeg $\mathrm{H}$ van de, Honing C. The general public's information needs and perceptions regarding hereditary cancer: an application of the Integrated Change Model. Patient Educ Couns. 2005 Feb;56(2):154-65.

50. Rejeski WJ, Tian L, Liao Y, McDermott MM. Social cognitive constructs and the promotion of physical activity in patients with peripheral artery disease. J Cardiopulm Rehabil Prev. 2008 Feb;28(1):65-72.

51. Caldieraro-Bentley AJ, Andrews JO. An integrative review: Application of self-efficacy instruments for walking in populations with peripheral arterial disease. J Vasc Nurs. 2013 Sep;31(3):118-30.

52. Collins TC, Lunos S, Ahluwalia JS. Self-efficacy is associated with walking ability in persons with diabetes mellitus and peripheral arterial disease. Vasc Med. 2010 Jun 1;15(3):189-95. 
сниттвк 10 Nederlandse samenvatting

Impactparagraaf

Dankwoord

Curriculum Vitae

List of publications

List of abbreviations 


\section{Nederlandse samenvatting}

De thesis geeft een inzicht in acute contrast geïnduceerde nierschade en langetermijneffecten van jodiumhoudend contrast op nierfunctie, evenals blootstelling aan ioniserende straling in de endovasculaire behandeling van de onderste extremiteiten. Ook is een alternatieve techniek beschreven in de behandeling van iliacale TASC II A en B-laesies en een manier om acute contrast geïnduceerde nierschade te voorkomen door furosemide geforceerde diurese met afgestemde intraveneuze hydratie. Tot slot zijn gedragscomponenten die van invloed zijn op de therapietrouw in gesuperviseerde looptherapie in kaart gebracht.

Het tweede hoofdstuk beschrijft de langetermijneffecten van jodiumhoudend contrast op de nierfunctie in patiënten die zich voor het eerst presenteren op de polikliniek met Rutherford klasse II en III perifeer arterieel vaatlijden. Patiënten werden behandeld met gesuperviseerde looptraining of een endovasculaire interventie. Veranderingen in eGFR (estimated glomerular filtration rate) werden na een jaar vergeleken tussen de twee verschillende interventiegroepen. Resultaten toonden een klinisch relevante en significante lange termijn verlies van nierfunctie na een endovasculaire interventie tegen perifeer arterieel vaatlijden in vergelijk met de controlegroep.

Voortbordurend op het voorgaande hoofdstuk wordt in hoofdstuk drie de langetermijneffecten van jodiumhoudend contrast op de nierfunctie in patiënten met kritieke ischemie (Rutherford klasse IV-VI) bestudeerd die of een endovasculaire interventie of een open chirurgische interventie ondergingen. eGFR na een jaar werd vergeleken, evenals het aantal patiënten welke een versnelde achteruitgang in nierfunctie ervaarden ( $>4 \mathrm{ml} / \mathrm{min}$ binnen een jaar). Resultaten toonden permanente lange termijn verlies in nierfunctie na een endovasculaire interventie in de behandeling van kritieke ischemie. Het verlies in nierfunctie was significant groter wanneer vergeleken met patiënten die een open chirurgische interventie ondergingen.

Hoofdstuk vier geeft een inzicht in de blootstelling aan ioniserende straling bij de endovasculaire behandeling van patiënten met symptomatisch perifeer arterieel vaatlijden. In dit deze observationele cohortstudie werden DAP (dose area product), PSD (peak skin dose), fluoroscopie tijd en volume van gebruikt contrastmedium gemeten of berekend. Resultaten werden gestratificeerd aan de hand van patiëntkarakteristieken en anatomisch locatie van de interventie. Het hoofdstuk toont aan dat ondanks een kortere fluoroscopie tijd er een grotere blootstelling aan straling is in aorta-iliacale interventies, wanneer wordt vergeleken femoro-popliteale of infra-popliteale interventies. 
In de zoektocht naar een preventieve maatregel tegen acute contrast geïnduceerde nierschade en de effecten van ioniserende straling werd een gerandomiseerd onderzoek opgezet, welke wordt besproken in hoofdstuk 5. Een relatief nieuwe applicatie van een reeds langer bestaande beeldvormende techniek werd ingezet in de behandeling van TASC II A en B-laesies op iliacaal door gebruik te maken van duplex. Technisch succes werd geëvalueerd waarbij duplex geleide PTA (DuPTA) werd vergeleken met conventionele PTA. De resultaten toonden aan dat de duplex geleide PTA een realistisch en noninferieur alternatief is in de behandeling van perifeer arterieel vaatlijden op iliacaal niveau. Overigens werd een significant grotere reductie in PSV (peak systolic velocity) bereikt bij gebruik van duplex geleide PTA.

De resultaten in hoofdstuk drie toonde aan dat $19 \%$ van de patiënten met Rutherford klasse IV-VI, die behandeld werden met een endovasculaire interventie, acuut contrast geïnduceerde nierschade ontwikkelden. Om inzicht te krijgen in deze acute effecten van het jodiumhoudend contrast op de nierfunctie wordt in hoofdstuk zes de acute- en lange-termijn nierfunctie na duplex geleide PTA en conventionele (contrast gemedieerde) PTA in patiënten met iliacale TASC II A en B-laesies vergeleken. Echter werden er geen significante verschillen in regressie van nierfunctie geconstateerd. Daarbij ontwikkelde geen van de patiënten een significante reductie in eGFR.

Endovasculaire behandeling van patiënten met perifeer arterieel vaatlijden wordt vaak gecompliceerd door comorbiditeiten zoals pre-existente chronische nierschade, waardoor ze vatbaarder zijn voor acute contrast geïnduceerde nierschade. Om op dit probleem te anticiperen ontvangen patiënten met chronische nierschade pre- en posthydratie. Echter wordt er vaak te voorzichtig en in een te laag volume gehydreert om een adequate bescherming te bieden, uit angst voor overvulling en pulmonaal oedeem. Hoofdstuk 7 omvat een studieprotocol aangaande de Renalguard, welke ons in staat stelt om de toe te dienen volume af te meten aan furosemide geforceerde diurese ten tijde van de endovasculaire interventie. Zodoende kan er een constante verhoogde perfusie over de nieren worden gegenereerd, zonder risico op overvulling, longoedeem of volumedepletie. Om een valide alternatief te bieden in de diagnostiek naar acute contrast geïnduceerde nierschade worden volgens het studieprotocol ook biomarkers in de urine gemeten, vier uur na de endovasculaire procedure. Hierdoor willen wij in een vroeger stadium de potentieel gedane nierschade vaststellen, gezien de gouden standaard middels serum creatinine 48-72 uur nodig heeft om een significante stijging te laten zien. 
Waar de vorige hoofdstukken de mogelijke negatieve gevolgen van endovasculaire interventies en potentiele alternatieve technieken beschrijven is het devies nog altijd; 'voorkomen is beter dan genezen'. Patiënten die zich voor het eerst presenteren op de polikliniek heelkunde met perifeer arterieel vaatlijden komen vaak met klachten van claudicatio intermittens. De eerste stap in de behandeling, gebaseerd op internationale richtlijnen, is om gesuperviseerde looptraining voor te schrijven. Ook al laten studies een significante toename zien in pijnvrije loopafstand en uitgestelde aanvang van de claudicatie, is de therapietrouw bij gesuperviseerde looptherapie laag. In hoofdstuk 8 worden de gedragsdeterminanten geïdentificeerd welke bijdragen aan de lage therapietrouw. Een vragenlijst werd opgesteld met gebruik van het I-CHANGE model, welke werd verzonden naar 200 patiënten die na initiële presentatie met claudicatio intermittens gesuperviseerde looptraining was voorgeschreven. Patiënten die hun loopafstand hadden vergroot na gesuperviseerde looptraining hadden een significant betere kennis, positieve attitude en lage negatieve attitude ten opzichte van gesuperviseerde looptraining. Het verbeteren van de determinanten kennis, attitude en de eigen overtuiging dat je in staat bent je gedrag te veranderen (self-efficacy) kunnen leiden tot een verbeterde therapietrouw en dus verbetering van de pijnvrije loopafstand. Hierdoor kan de noodzaak om over te gaan op een chirurgisch interventie worden voorkomen of uitgesteld. 


\section{Impactparagraaf}

1. (Research) What is the main objective of the research described in the thesis and what are the most important results and conclusions?

The main objective of the research in this thesis is to get an insight and quantify side effects of several treatment strategies regarding peripheral arterial disease (PAD). Moreover, how can we anticipate or abolish these side effects. For instance; endovascular treatment of PAD requires the use of iodine contrast and radiation to image arteries, which are damaging to several organs, such as skin and kidneys. Therefore, first line treatment of PAD on initial presentation is supervised exercise therapy (SET), whereas this is a non-invasive strategy. We demonstrated that renal function after one year declined significantly more in patients treated with an endovascular procedure, when compared with SET. Also, long-term renal function is significantly worse in patients treated with an endovascular intervention when compared to open surgery. The worsening renal function is a supposed consequence of iodine contrast. Moreover, radiation exposure is known to cause potential acute and/ or long-term effects on penetrating tissue (skin, muscle). To get a better understanding of which anatomical region is most at risk we quantified the used radiation doses and cumulative time of radiation in several procedures. This thesis showed that the radiation exposure is highest in arterial treatment of arteries in the pelvic area and the thigh, when compared to the more distal regions. The more tissue to penetrate imaging the arteries, the higher the radiation dose.

To address these issues, we investigated ways to decrease the detrimental effects and studied novel approaches to completely eliminate these effects, without compromising in outcome. We studied the possibility of treating a clothed artery in de legs with balloon dilatation using ultrasound as an imaging technique. The results showed no difference in success compared to the conventional imaging technique using contrast and radiation. Furthermore, this thesis quantified renal function before and after an intervention. No significant decrease in renal function was observed in either treatment group. This is possibly explained by adequate fluid therapy, improvement in imaging techniques and iodine contrast. However, patients with poor renal function before an intervention are at increased risk of renal decline after the use of iodine contrast. Using fluid therapy, concentration of iodine contrast in the kidneys is diluted. Preferably a high and steady urine production is achieved, without risking excess fluid administration. To achieve high urine output, diuretics can be administered. However, there is an increased risk of 
fluid depletion and thus increasing iodine contrast concentration and possible damage to the kidneys. To achieve the right balance of administered fluids and diuretics is difficult. Therefore, we designed a study protocol using the Renalguard. This is a little device which weights the urine produced and administers fluids accordingly to achieve an increased and steady flow over the kidneys. This study will examine the benefit of the Renalguard compared to conventional fluid therapy in patients treated endovascularly for PAD.

Finally, as mentioned earlier SET is the initial treatment regarding intermittent claudication. However, even though great improvement of walking distance can be achieved when dedicated to the therapy, adherence to SET is low. We tried to identify reasons for low adherence and identified several relevant behavioral determinants. It appeared knowledge, attitude and self-efficacy are of great importance and need to be addressed to improve adherence to SET.

2. (Relevance) What is the (potential) contribution of the results from this research to science, and, if applicable, to social sectors and social challenges?

The contribution of the results from this science is plural. It gives an insight in the longterm effects of endovascular procedures for intermittent claudication and critical limb ischemia on renal function. Furthermore, we determined radiation exposure in different anatomical locations of patients treated with endovascular interventions of the lower extremities. We provided a good alternative for endovascular treatment of the groin area in patients with diminished renal function without having to rely on contrast agent. The results disputed the arguments of any hindrance of duplex visualization due to bowel gas or obesity. The research also suggests a possible solution in furosemide forced diuresis, which also limits the harmful effects of iodine contrast on kidneys. To prevent an invasive intervention altogether, physicians need to increase a patient's knowledge and attitude toward PAD and SET. This might aid physicians and patients to improve adherence to SET and avert an invasive procedure.

\section{3. (Target group) To whom are the research results interesting and/or relevant? And why?}

The research results are mainly interesting and relevant for vascular surgeons and interventional radiologists treating patients with PAD in the lower extremities. Results might also be relevant for general practitioners, in particular how to approach a patient on initial presentation with intermittent claudication. The general practitioner often has a closer and more frequent contact with a patient. This might result in a greater influence on a patient's behavior and thus be more inclined to take the general physicians advise. 
4. (Activity) In what way can these target groups be involved in and informed about the research results, so that the knowledge gained can be used in the future?

These target groups are and can be informed by presenting these study results on national and international conferences. Furthermore, the studies in this research are published in international journals. 


\section{Dankwoord}

Het dankwoord. Ik schrijf nog liever een artikel. Waar het voor de een de kers op de taart is, is het voor mij de molensteen om mijn nek. Desalniettemin misschien wel het belangrijkste hoofdstuk van je proefschrift en de kans even stil te staan bij wat de mensen om je heen voor je betekenen. Where to begin.. Ken je dat moment dat je de slaap nog net niet kan vatten, het meest creatieve moment van de dag? Dochter ligt inmiddels alweer tussen ons in, slapend en rustig in een steady ritme haar voetjes in mijn nieren te planten. Zucht.., er toch maar even uit, schemerlampje aan, laptop open. Zit je momenteel thuis met een positieve coronatest, zo 2022. Het dankwoord, zo lang uitgesteld. Maar ja, there is no better moment than the present. Here we go!

Het heeft wat voeten in de aarde gehad. Ruim 6 jaar heb ik nodig gehad om mijn proefschrift tot een succesvol einde te kunnen brengen. Alleen had ik dit nooit kunnen volbrengen. Mede dankzij een vliegende start (dankjewel Tim Sigterman) en enkele nader te noemen belangrijke mensen heb ik dit kunnen presteren. Door verandering in mijn professionele ambities en privésituatie was het onderzoek op een zijspoor geraakt. Soms weer een opleving rondom een publicatie en motiverende gesprekken met Lee Bouwman. Ik ben er enorm trots op dat ik het af heb kunnen maken, trots op mijzelf maar ook als voorbeeld naar mijn kinderen. Als je ergens aan begint maak je het ook af. Om Brad Pitt te quoten in de film Snatch; "You stay until the job's done!'.

Als eerste wil ik mijn promotieteam bedanken, prof. dr. GWH Schurink en dr. LH Bouwman. Beste Geert Willem, bedankt voor het delen van je ervaring in het wetenschappelijk onderzoek. Altijd bereid constructief mee te denken in het onderzoek en het schrijven van de artikelen. Door jouw bijdrage hebben we dit proefschrift tot een hoger niveau weten te tillen. Beste Lee, ik ben je zeer dankbaar voor je actieve betrokkenheid tijdens mijn tijd bij de heelkunde. Ik heb me altijd zeer gesteund gevoeld en ben blij om jou als zwaargewicht, in mijn hoek te hebben staan. Ik ben me er altijd zeer bewust van geweest dat jij je nek voor mij hebt uitgestoken binnen de maatschap heelkunde. Dit maakte voor mij de keuze om niet te solliciteren voor een AIOS-plek bij de heelkunde des te lastiger. Ook het vertellen van deze beslissing vond ik zeer lastig, niet wetende hoe je dit zou oppakken. In plaats van teleurstelling heb je mij gesteund en gesterkt in mijn beslissing en daarbij persoonlijke gevoelens opzijgezet. Lee, je bent voor mij een groot voorbeeld als arts, maar vooral ook als mens. Go raibh maith agat! 
Attila, immer ontspannen en goed gehumeurd. Bedankt voor het delen van je kennis en kunde, met name op het gebied van de veneuze vaatchirurgie. Heb altijd prettig met je samengewerkt. Ben je me toch nog qua promotie voorgebleven! Kees Jan, de kritische noot. Blijf altijd zelf nadenken en wees kritisch op je eigen functioneren. Altijd stimulerend om je te blijven ontwikkelen.

Zoals eerdergenoemd wil ik graag mijn paranimf Tim Sigterman bedanken. Door jou kwam ik met mijn promotietraject in een gespreid bedje. Ook in jouw tijd als ANIOS en later AIOS heb je nog veel steun kunnen bieden met de reeds lopende onderzoeken. Daarbij heb je ook altijd meegedacht en gewerkt aan nieuwe onderzoeksprotocollen en artikelen. Jouw ervaring en discipline hebben een grote bijdrage geleverd een mijn uiteindelijke manuscript. Daarbij kan ik Rochelle natuurlijk niet vergeten! Ook altijd bereid om belangeloos mee te denken en mij wegwijs te maken in de wetenschappelijke wirwar en statistiek. Jullie zijn toppers, bedankt.

De mogelijkheid om te promoveren heb ik uiteraard ook te danken aan de Maatschap Heelkunde, 5 Jaar lang hebben jullie mij onder jullie hoede genomen. Eerst als semi-arts, later als promovendus en tot slot als ANIOS heelkunde. Ik heb veel bij jullie kunnen leren qua kennis maar bovenal ook het arbeidsethos 'niet lullen maar poetsen'. Al was ik niet bepaald een van de gangmakers onder de assistenten, ik heb mij wel altijd gewaardeerd gevoeld. Daarbij hebben uiteraard ook de polimedewerkers een belangrijke bijdrage geleverd. Gezelligheid voorop, altijd meedenken en een handje hulp wanneer nodig. Bedankt voor jullie bijdrage aan mijn plezierige tijd bij de heelkunde. Niet te vergeten wil ik ook Simone, Marlies, Ria en de dames van het secretariaat bedanken.

Mijn vaatbuddies op de poli Ed, Anouk, Cleo, Monique en Mike uiteraard ook bedankt voor de prettige samenwerking. Jullie denken altijd in oplossingen in plaats van problemen. Wanneer nodig wordt er gewoon een tandje bij gezet. Ik heb veel van jullie mogen leren. Met name wil ik jullie bedanken voor jullie bijdrage aan het onderzoek en bijspringen wanneer nodig. Eveneens cruciaal voor mijn onderzoek zijn de interventieradiologen Roel Heijboer, Bernard de Leeuw en alle angiolaboranten. Ik kon altijd bij jullie even binnenlopen en door de logboeken struinen voor aanvullende informatie voor mijn databases. Met name dank aan Jacques welke zijn bijdrage heeft geleverd aan het selecteren van de afbeelding op de cover van dit proefschrift.

Na mijn tijd bij de heelkunde ben ik een ander pad ingeslagen bij de huisartsgeneeskunde. 
Voor mij de beste keus, toen en nu nog steeds. Een nieuwe plek om te groeien en een goede fit voor mij als persoon en als arts. Zodoende wil ik mijn opleiders en studiegenoten bij de huisartsgeneeskunde bedanken. Allereerst Gaston en Marieke. Zij hebben bijgedragen aan een soepele start van de opleiding, mij geënthousiasmeerd voor het vak en mij vertrouwen gegeven. Daarbij natuurlijk ook de groepsgenoten van jaar 1 voor de gezellige terugkomdagen en oprechte interesse voor mijn onderzoek en mij als persoon. Een special shout-out naar Priscilla Mantingh en Yvonne Nooijen, een betere eerstejaarspraktijk kon ik mij niet wensen. Zo een fijn team. Jullie hebben een zeer grote bijdrage geleverd aan wie ik ben, en wordt als arts. Ik ben elke dag met plezier naar de praktijk gekomen en heb nog het een en ander geleerd over in de stront roeren! $\mathrm{Nu}$ inmiddels bezig aan jaar drie van de opleiding en ook hier getroffen met mijn groepsgenootjes en opleiders. We zijn nog maar enkele maanden onderweg maar voel mij al prima op mijn plek. Lekker klagen over hoe zwaar het wel niet is als verse ouders, gedeelde smart is halve smart! Ook mijn 3de jaar praktijk is een schot in de roos. Jeroen Smeets met zijn onuitputtelijke energie wil ik dan ook bedanken voor de begeleiding tijdens mijn laatste jaar in de opleiding, de ruimte die ik krijg om het maximale eruit te halen en de tijd om mijn proefschrift tot een mooi einde te brengen.

Voorzitter en leden van de beoordelingscommissie, Prof. dr. J.W.M. Muris (voorzitter), Prof. dr. A.W.J. van 't Hof, Prof. dr. J.A. van Herwaarden en Dr. J. Heyligers, hartelijk dank voor de kritische beoordeling van mijn proefschrift en deelname aan de corona.

Waar eerder al Tim genoemd werd als een van de paranimfen moet ook zeker Mark Thijssen niet worden vergeten. Jij werd door Kirsten schaamteloos ingezet als soort van mediator om het eerste contact tussen ons te leggen. Hierin ligt meteen ook de basis van onze vriendschap, inmiddels alweer 12 jaar geleden. Later kwam daar uiteraard ook Stephanie bij. Inmiddels gaan we al jaren met elkaar op wintersport en waren jullie aanwezig bij ons huwelijk in Toscane. Mark, je bent een van de meest oprechte en betrouwbare mensen die ik ken en ben je dankbaar dat ik je een vriend mag noemen.

$\mathrm{Nu}$ ik bij de vrienden ben aangekomen wil ik ook graag even stil staan bij de twee Groninger pinda's. Apa kabar? George-Emile, we go way back, reeds in de zandbak op 3-jarige leeftijd waren wij twee handen op een buik. Struinend door Stedum hebben wij heel wat avonturen beleeft. Helaas is het contact iets minder frequent na je vertrek richting Seattle, maar nu je weer naar ons kikkerlandje terugkomt hoop ik weer meer van je te zien! Mick, de ander pinda. Ook jou heb ik helaas al even niet kunnen zien. Inmiddels alweer 
enkele jaren woonachtig op Bali, de plek waar wij ook samen goede tijden hebben beleefd. Ik kijk dan ook zeer uit om je daar weer te ontmoeten en oude tijden te herleven! Ik hoop dat tegen die tijd ook dit proefschrift je goed heeft weten te bereiken!

OMHD e Causa Ignota, mooie mannen. Ook jullie kan ik natuurlijk niet vergeten. Veel van mijn mooiste momenten uit mijn studententijd heb ik met jullie mogen doormaken. Hoe mooi is het dat ik mijn proefschrift mag verdedigen op onze oprichtingsdatum!

Jan en Ina, lieve ouders, bedankt voor jullie geduld en onvoorwaardelijke steun en liefde. Ik was niet altijd de makkelijkste en derhalve hebben wij soms wel onze moeizame momenten gekend. De basis was echter goed, dit gaf mij de ruimte om de ontdekken en onderzoeken. Door dik en dun weet ik dat jullie er altijd voor mij zullen zijn, zo zal ik er ok altijd voor jullie zijn. Bedankt.

Joost, Ruben, Ivan en Margje, broers en zus. Als samengeraapt zootje denk ik dat we het toch allemaal op onze eigen wijze goed doen. Juist dat wij allen zo verschillend zijn heeft bijgedragen aan mijn persoonlijke ontwikkeling en heeft geholpen mij te vormen tot wie ik nu ben. Helaas maakt de afstand het vaak lastig om elkaar frequent te zien, als we dan wel weer bij elkaar komen voelt het als een warm bad. Groningen zal altijd als thuis voelen, juist omdat jullie daar zijn.

Lieve Kirsten, dit proefschrift had ik ook zeker niet kunnen volbrengen zonder jou. Altijd stimulerend en steunend in mijn beslissingen. Als rots in de branding ben jij mijn constante, degene waarover ik nooit hoef te twijfelen. Je bent er altijd voor mij en geeft me de ruimte waar nodig, zo ook om aan het proefschrift te werken. Zelfs meermaals op vakantie toch nog de laptop er even bij pakken voor het indienen van een artikel of maken van revisies. Daarnaast als moeder van onze kinderen hebben ook zij het niet beter konden treffen. Ook voor hen een stabiele factor met liefde ten overvloede. Lieve Kirsten, bedankt.

Mads, Mette en ..., jullie zullen je later niet meer kunnen herinneren dat jullie papa af en toe even minder beschikbaar was omdat er gewerkt moest worden aan dit proefschrift. Maar jullie zijn juist een van de voornaamste redenen dat ik dit proefschrift toch heb kunnen afronden. Ik wil jullie graag meegeven dat ondanks af en toe tegenslagen of gebrek aan motivatie, het goed is om niet bij het minste of geringste op te geven maar je zinnen te verzetten en door te zetten. Probeer af te maken waar je aan begint. "The greater the effort, the sweeter the reward". 
Tot slot zijn er altijd nog de mensen die niet genoemd, maar zeker niet minder belangrijk zijn. Tijdens het onderzoek ben ik met veel mensen in aanraking gekomen. Ook jullie bedankt voor jullie bijdrage aan het onderzoek.

Moet je kijken, is het me toch gelukt, en heb ik er ook nog plezier in gehad ook.. 


\section{Curriculum Vitae}

Lars Bolt was born on the $8^{\text {th }}$ on May 1987 in Groningen. After finishing secondary school at H.N. Werkman college in Groningen, he studied biomedical sciences for a year at the university of Groningen. In 2009 he started medical school at the university of Maastricht. During his bachelor he worked as student-assistant at the university of Maastricht and successfully participated in the research honours program. His clinical internships included Opthalmology in Pretoria, South Africa and Pediatrics in Palembang, Indonesia. In his last year he started his

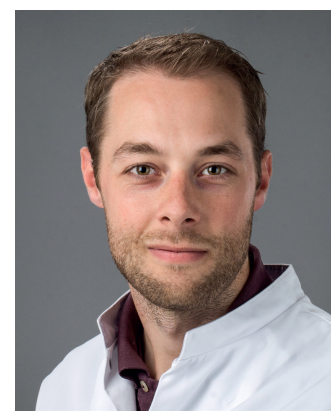
research with dr. L.H. Bouwman and dr. T.A. Sigterman, eventually resulting in this thesis. After obtaining his medical degree (MD) in 2015 he started working as a PhD student with Prof. dr. GW. Schurink (Vascular surgeon, Maastricht University Medical Center) as his promotor and dr. L.H. Bouwman (Vascular surgeon, Zuyderland, Medical Center) as his co-promotor. During this period, he presented his research findings on national and international congresses. From 2017 till 2019 he worked as a surgical resident not in training in Zuyderland Medical Center. In his spare time, he continued his research. After deciding not to pursue a surgical career he worked for a short period as a doctor not in training in a geriatric rehabilitation center (Meander). In 2020 he started his training as general practitioner and is expected to finish in 2022. 


\section{Bibliography}

Renal decline after endovascular intervention for peripheral artery disease: iodine contrast PTA vs. duplex-guided PTA in iliac stenotic disease. LJJ. Bolt, TA. Sigterman, AG. Krasznai, CJJM. Sikkink, GWH. Schurink. LH. Bouwman. To be submitted

Assessment of behavioral determinants influencing success of supervised exercise therapy in patients with intermittent claudication: A cross sectional survey. LJJ. Bolt, MLYE. Jacobs, TA. Sigterman, AG. Krasznai, CJJM. Sikkink, GWH. Schurink, LH. Bouwman. Physiol Behav. 2020 Mar 1;215:112732.

Prevention of surgical site infections using gentamicin-containing collagen implants following inguinal incision for vascular surgery: A prospective randomized controlled multicenter trial. L. Wubbeke, T. van Boxtel, L. Bolt, B. Telgenkamp, S. Houthoofd, P. Vriens, L. Bouwman, B. Mees, J.W. Daemen. Eur J Vasc Endovasc Surg. 2019 Dec; 58: e726

Prevention of postcontrast acute kidney injury after percutaneous transluminal angioplasty by inducing RenalGuard controlled furosemide forced diuresis with matched hydration: study protocol for a randomised controlled trial. Bolt LJJ, Sigterman TA, Krasznai AG, Sikkink CJM, Schurink GH, Bouwman LH. BMJ Open. 2018 Oct 4;8(9):e021842.

Duplex-guided versus Conventional Percutaneous Transluminal Angioplasty of Iliac TASC II A and B Lesion: A Randomized Controlled Trial. Bolt LJJ, Krasznai AG, Sigterman TA, Sikkink CJJM, Schurink GWH, Bouwman LH. Ann Vasc Surg. 2018 Oct 1. pii: S08905096(18)30777-5.

Radiation Exposure during Percutaneous Transluminal Angioplasty for Symptomatic Peripheral Arterial Disease. Sigterman TA, Bolt LJ, Snoeijs MG, Krasznai AG, Heijboer R, Schurink GW, Bouwman LH. Ann Vasc Surg. 2016 May;33:167-72

Loss of kidney function in patients with critical limb ischemia treated endovascular or Surgically. Sigterman TA, Bolt LJ, Krasznai AG, Snoeijs MG, Heijboer R, Schurink GH, Bouwman LH. J Vasc Surg. 2016 Aug;64(2):362-368 
Epidemiology, management and survival outcomes of primary cutaneous melanoma: a ten-year overview. Aubuchon MM, Bolt LJ, Janssen-Heijnen ML, Verleisdonk-Bolhaar ST, van Marion A, van Berlo CL. Acta Chir Belg. 2016 Oct 23:1-7

Loss of Kidney Function after Endovascular Treatment of Peripheral Arterial Disease. Sigterman TA, Bolt LJ, Krasznai AG, Snoeijs MG, Heijboer R, Schurink GW, Bouwman LH. Ann Vasc Surg. 2016 Nov 27. pii: S0890-5096(16)31256-0 


\section{List of abbreviations}

\begin{tabular}{ll} 
ABI & Ankle-Brachial-Index \\
AERD & ArtheroEmbolic Renal Disease \\
AKI & Acute Kidney Injury \\
ALARA & As Low As Reasonably Achievable \\
ALI & Acute Limb Ischemia \\
BMI & Body Mass Index \\
CFA & Common Femoral Artery \\
CHF & Chronic Heart Failure \\
CIA & Common Iliac Artery \\
CIN & Contrast induced Nephropathy \\
CKD & Chronic Kidney Disease \\
CLI & Critical Limb Ischemia \\
COT & Claudication Onset Time \\
CRFs & Case Report Forms \\
CTA & Computed Tomography Angiography \\
DAP & Dose Area Product \\
DM & Diabetes Mellitus \\
DuPTA & Duplex guided Percutaneous Transluminal Angioplasty \\
eGFR & estimated Glomerular Filtration Rate \\
EIA & External Iliac Artery \\
ELISA & Enzyme-Linked ImmunoSorbent Assay \\
ESRD & End-Stage Renal Disease \\
EVAR & Endovascular Aneurysm Repair \\
FPS & Frames Per Second \\
GFR & Glomerular Filtration Rate \\
IC & Intermittent Claudication \\
ICM & I-Change Model \\
IL-18 & Interleukin-18 \\
KIM-1 & Kidney Injury Molecule-1 \\
L-FABP & Liver Fatty Acid Binding Protein \\
MI & Myocardial Infarction \\
MRA & Magnetic Resonance Imaging \\
NAG & N-acetyl-beta-D-glucosaminidase \\
NGAL & Neutrophil Gelatinase-Associated Lipocalin \\
& \\
\hline
\end{tabular}


NRCP National Council on Radiation Protection and Measurements

NSF

Nephrogenic Systemic Fibrosis

PAD Peripheral Arterial Disease

PC-AKI Post Contrast Acute Kidney Injury

PI Principle Investigator

PSD Peak Skin Dose

PSV Peak Systolic Velocity

PTA Percutaneous Transluminal Angioplasty

PWD Peak Walking Distance

PWT Peak Walking Time

RCT Randomized Controlled Trial

REC Research Ethics Committee

SET Supervised Exercise Therapy

TASCII Trans-Atlantic Inter-Society Consensus Document II

TBI Toe-Brachial-Index

TIMP-2 Tissue Inhibitor of MetalloProteinase-2 



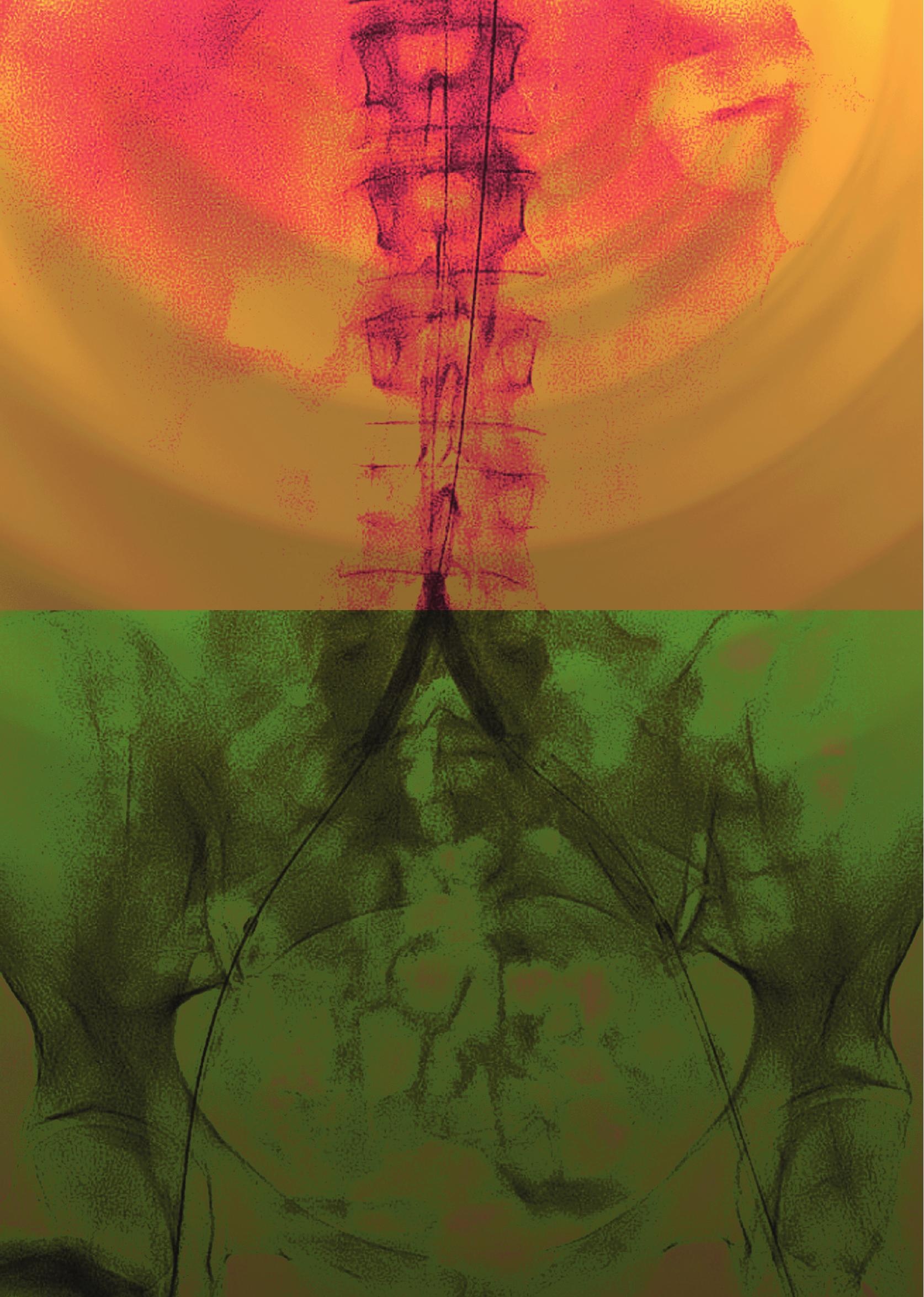

